\title{
A Law of Large Numbers in the Supremum Norm for a Multiscale Stochastic Spatial Gene Network
}

\author{
${ }^{1}$ Institut de Recherche Mathematique de Rennes, Rennes, Bretagne, France, E-mail: nguepedja.mac-jugal@ens-rennes.fr
}

\begin{abstract}
:
We study the asymptotic behavior of multiscale stochastic spatial gene networks. Multiscaling takes into account the difference of abundance between molecules, and captures the dynamic of rare species at a mesoscopic level. We introduce an assumption of spatial correlations for reactions involving rare species and a new law of large numbers is obtained. According to the scales, the whole system splits into two parts with different but coupled dynamics. The high scale component converges to the usual spatial model which is the solution of a partial differential equation, whereas the low scale component converges to the usual homogeneous model which is the solution of an ordinary differential equation. Comparisons are made in the supremum norm.
\end{abstract}

Keywords: probabilities, stochastic processes, biochemical modeling, Epidemiology

DOI: 10.1515/ijb-2017-0091

Received: November 14, 2017; Revised: February 14, 2019; Accepted: April 10, 2019

\section{Introduction}

Modern molecular biology emphasizes the important role of gene regulatory networks in the functioning of living organisms (see [16], [41] or [33]). As many other dynamical systems in a great variety of fields (e.g. biology, chemistry, epidemic theory or physics), gene regulatory networks belong to the large family of chemical reaction systems, which has been highly investigated in mathematics, for modeling. We refer to [30, 31], [3], [2], [17], [18], [37], [13], [12], and many other references therein. Following Arnold in [2], there are two main criteria according to which reactions in a spatial domain are modeled:

(C1) global description (spatially homogeneous, "well-stirred" case excluding diffusion) versus local description (spatially inhomogeneous, "spatial model" including diffusion);

(C2) deterministic description (macroscopic, phenomenological, in terms of concentrations) versus stochastic description (mesoscopic, taking into account fluctuations, at the level of particles or molecules).

The combination of these two criteria gives rise to four mathematical models:

- Deterministic-Homogeneous: the concentration is solution to an Ordinary Differential Equation (ODE).

- Stochastic-Homogeneous: the number of particles is a (time) jump Markov process.

- Deterministic-Spatial: the concentration is solution to a Partial Differential Equation (PDE).

- Stochastic-Spatial: the number of particles is a (space-time) jump Markov process.

There is a restrictive assumption for deterministic and many of the stochastic models, in the literature. Namely, the different species (or reactants) have the same order of population size, which is large, meaning that the whole system has a fast dynamic. There are many biological - or not - situations for which this is not true, where different scales of population sizes and speeds of dynamics - fast and slow - are coupled. Gene regulatory networks are typical situations, where some molecules are present in much greater quantity than others, and reactions rate constants can vary over several orders of magnitude (see [12], [5]). It is also the case in neurosciences (see [10,21,38] based on Hodgkin-Huxley's model for the propagation of action potentials in neurons), in systems pharmacology (see [32] for drug effects for cancer treatment), in population dynamics (see [4] for biological invasions).

A third criterion of modeling then clearly emerges:

(C3) one-scale description with a unique and large population size scale, with only high reaction rates and fast dynamics, versus multiscale description with at least two population size scales, with high and low reaction rates, and, fast and slow dynamics.

Now, with the three criteria (C1), (C2), and (C3) in hands, we can derive: the

Mac Jugal Nguepedja Nankep is the corresponding author

(c) 2019 Walter de Gruyter CmbH, Berlin/Boston.

This content is free. 
(M1) Deterministic Homogeneous Model,

(M2) Deterministic Spatial Model,

(M3) Stochastic Homogeneous Model,

(M4) Stochastic Spatial Model,

(M5) Multiscale Stochastic Homogeneous Model,

(M6) Multiscale Stochastic Spatial Model.

It should be emphasized that criterion (C3) has been introduced at the mesoscopic level and thus suggests to preferentially consider a stochastic description for multiscaling. This stresses the importance of, and our focus on, the multiscale stochastic modeling.

All these models are not independent one from another. The relation between (M1) and (M3) has been thoroughly investigated by Kurtz (among others, see [30,31] or [17] with Ethier). A law of large numbers (LLN) and the corresponding central limit theorem (CTL) have been proved, showing convergence of (M3) to (M1). The consistency of (M1) and (M2) as well as of (M3) and (M4) have been proved in [2]. In [3], Arnold and Theodosopulu compared (M2) and (M4) in the $L^{2}$ norm, through a LLN. Blount after Kotelenez did the same comparison, but much more extensively. Under more and more relaxed assumptions, they proved different LLNs and the associated CLTs in spaces of distributions. Blount showed a LLN in the supremum norm. We refer to [24-27], [6-9] among others.

In [37], [13] and [12], Crudu, Debussche, Muller and Radulescu studied (M5). In the latter article, they integrated the multiscale nature of the system in their modeling approach and proved that the multiscale model (weakly) converges to a finite dimensional Piecewise Deterministic Markov Process (PDMP). In finite dimension, PDMPs are hybrid processes which follow between consecutive jumps the flows of ODEs whose parameters can jump. They have been well formalised and studied by Davis in [15]. Depending on the interactions and the scaling, [12] distinguished various types of limiting PDMPs. Stochasticity does not disappear in that hybrid simplification, and multiscaling appears to be a tool for estimating asymptotically at the first order, the noise lost in the LLN.

Direct simulation of Markov processes is extremely time consuming for models we are dealing with (see [13] or [22]). The finite dimensional hybrid simplification made in [37], [13] and [12] allows to consider PDMPs instead of the initial multiscale model for simulations and analysis. This justifies, in the homogeneous context, the already common use of hybrid model and accelarated algorithms which considerably increase computational efficiency. Lately, this practice has been extended to spatially inhomogeneous systems, leading to the simulation of multiscale spatial stochastic systems as infinite dimensional hybrid systems. We can cite [1] for cell regulatory networks and enzyme cascades, [34] for molecular communication or [10, 21, 38] in neurosciences. It is therefore important to justify mathematically the use of these hybrid approximations for multiscale spatial systems and to understand whether they are always consistent, or not. This is the aim of this article.

In this article, we initiate the study of (M6) and generalize the approach of [12] to a spatially dependent situation. Depending on their abundance, we distinguish abundant ("continuous") and rare ("discrete") species. Also, there are different speeds of reactions, according to their rate scale and the species involved. When only abundant (resp. rare) species are involved, reactions are fast (resp. slow). Otherwise, the speed depends on the specification of the rate scale. We assume that only abundant reactants diffuse. Also, a spatial correlation for slow reactions is considered - we explain below why it is necessary to introduce such correlation. Its effects are inversely proportional to the distance to the location where reactions occurred. In this context, we prove a new law of large numbers, showing the convergence of (M6) to (M2) coupled with (M1), in the supremum norm. This shows that, contrary to the normal intuition and to what happens in the homogeneous case, stochasticity disappears even though there are several scales. Therefore, one must be very careful before using multiscale spatial stochastic simplified systems. In a forthcoming work, we introduce another situation in which the discrete species remain at the macroscopic level and thus the multiscale character is both in time and space. In this situation, we obtain a PDMP consisting of reaction-diffusion equations whose coefficients change at some jumps.

The structure of the article is as follows. In Section 2, we present local models, starting with the existing ones. We emphasize the notions of infinitesimal generator, debit functions, scaling and density dependence. Then we present our model of interest (M6). Afterwards, we identify its limit through the asymptotics of its debit function and of its generator. We also state our main result in Section 2. Future works and how our results can be used for statistical analysis are indicated. The main result is proved in Section 3. An appendix is devoted to the proof of technical results used in Section 3.

Some general notations. Let $\left(Z,\|\cdot\|_{Z}\right)$ and $\left(\tilde{Z},\|\cdot\|_{\tilde{Z}}\right)$ be Banach spaces. The product space $Z \times \tilde{Z}$ is equipped with the norm $\|\cdot\|_{Z}+\|\cdot\|_{\tilde{Z}}$. We introduce: 
- $\mathscr{L}(Z, \tilde{Z})$ : the space of continuous linear maps from $Z$ to $\tilde{Z}$. If $Z=\tilde{Z}$, one simply writes $\mathscr{L}(Z)$. The operator norm is denoted $\|\cdot\|_{Z \rightarrow \tilde{Z}}$ and when there is no risk of confusion, we denote it $\|\cdot\|$.

- $Z^{\prime}$ : the space of continuous linear forms on $Z$. It is the topological dual space of $Z$.

$-\mathfrak{B}(Z)$ (resp. $\left.\mathfrak{B}_{b}(Z)\right)$ : the space of Borel-measurable (resp. bounded Borel-measurable) real valued functions on $Z$. The space $\mathfrak{B}_{b}(Z)$ is endowed with the supremum norm

$$
\|f\|_{\mathfrak{B}_{b}(Z)}=\sup _{x \in Z}|f(x)|=\|f\|_{\infty} .
$$

- $C_{b}^{k}(Z), k \in \mathbb{N}$ : the space of real valued functions of class $C^{k}$ on $Z$, i. e. $k$-continuously Fréchet differentiable on $Z$, which are bounded and have uniformly bounded succesive differentials. It is equipped with the norm

$$
\|f\|_{C_{b}^{k}(Z)}=\sum_{i=0}^{k}\left\|D^{i} f\right\|_{\infty}
$$

where $D^{i} f$ is the $i$-th differential of $f \in C_{b}^{k}(Z), C_{b}^{0}(Z)=C_{b}(Z)$ is the set of bounded continuous real valued functions on $Z$.

- $C^{l, k}(Z \times \tilde{Z}), l, k \in \mathbb{N}$ : the set of real valued functions $\varphi$ of class $C^{l}$ with respect to (w.r.t.) the first variable and of class $C^{k}$ w.r.t. the second. In particular, $C^{0,0}(Z \times \tilde{Z})=C(Z \times \tilde{Z})$.

For $(z, \tilde{z}) \in Z \times \tilde{Z}$, we denote by $D^{l, k} \varphi(z, \tilde{z})$ the (Fréchet) differential of $\varphi$, of order $l$ w.r.t. $z$ and of order $k$ w.r.t. $\tilde{z}$, computed at $(z, \tilde{z})$.

Also, a subscript $b$ can be added - to obtain $C_{b}^{l, k}(Z \times \tilde{Z})$ - in order to specify that the functions and their succesive differentials are uniformly bounded.

$-C_{p}(I)$ : the set of piecewise continuous real valued functions defined on $I=[0,1]$. It is equipped with the supremum norm.

- $C\left(\mathbb{R}_{+}, Z\right)$ : the set of continuous processes defined on $\mathbb{R}_{+}$with values in $Z$.

- $D\left(\mathbb{R}_{+}, Z\right)$ : the set of càdlàg processes defined on $\mathbb{R}_{+}$and taking values in $Z$. It is endowed with the Skorohod topology.

\section{Modeling and asymptotics}

\subsection{One-scale spatial models}

Since we are not concerned with the homogeneous models, we only review the mathematical details of the local models as given by Arnold and Theodosopulu in [3].

\subsubsection{Deterministic spatial model}

One-scale chemical reactions with diffusion in a spatial domain $I \subset \mathbb{R}^{d}$ are modeled deterministically, at a macroscopic level, by the reaction-diffusion equation

$$
\frac{\partial v}{\partial t}=D \Delta v+R(v),
$$

where $v=v(t, x)$ is an $M$-dimensional real vector which gives the concentrations for the $M \geq 1$ reactants involved in the interactions, $x \in I$ is the spatial coordinate, $t \geq 0$ is the time. The domain $I$ can be bounded or unbounded, $v$ is subjected to boundary and initial conditions, $\Delta$ is the Laplace operator, $D$ is a diagonal matrix of size $M$, with non-negative coefficients on the diagonal, called the diffusion matrix. As it is often the case, we consider 
for simplicity $D=I_{d}$, the idendity matrix (in the rest of the article, $D$ is the differential operator). Reactions are represented by $R$, a polynomial vector field in $\mathbb{R}^{M}$, defined by

$$
R(y)=\left(R_{1}(y), \cdots, R_{M}(y)\right) \quad \text { with } \quad R_{i}(y):=\sum_{|\alpha| \leq n} a_{\alpha}^{i} y^{\alpha},
$$

where $n \in \mathbb{N}^{*}, \alpha=\left(\alpha_{1}, \cdots, \alpha_{M}\right) \in \mathbb{N}^{M}$ is a multi-index, $|\alpha|:=\alpha_{1}+\cdots+\alpha_{M}, a_{\alpha}^{i} \in \mathbb{R}, y=\left(y_{1}, \cdots, y_{M}\right) \in \mathbb{R}^{M}$ and $y^{\alpha}:=y_{1}^{\alpha_{1}} \cdots y_{M}^{\alpha_{M}}$.

Well-posedness, generator and debit function. For simplicity, we work on a one dimensional domain, $d=1$, that we project on the unit interval $I=[0,1]$ with periodic boundary conditions: $v(t, 0)=v(t, 1)$, for all $t \geq 0$. Since concentrations are positive quantities, a positive initial data is considered: for all $x \in I, v(0, x) \geq 0$ in the sense $v_{i}(0, x) \geq 0$, for all $1 \leq i \leq M$. In order to have some control on the concentrations, it is assumed that $v(0)=v(0, \cdot)$ is bounded: $v(0, x)<\rho_{1}<\infty$, for all $x \in I$, for some $\rho_{1}>0$, and that:

\section{Assumption 2.1}

(i) For all $y=\left(y_{1}, \cdots, y_{M}\right) \in \mathbb{R}^{M}, R_{i}(y) \geq 0$ when $y_{i}=0$ for some $1 \leq i \leq M$.

(ii) There exists $\rho_{2}>0$ such that $\langle R(y), y\rangle<0$ for all $y \in \mathbb{R}^{M}$ satisfying $|y|>\rho_{2}$.

We have denoted by $|\cdot|$ and $\langle\cdot, \cdot\rangle$ the vector norm and the inner product of $\mathbb{R}^{M}$ respectively. Assumption 2.1 is natural, and ensures the consistency of the model through a priori estimates for (1). In fact, (i) yieds positiveness: $v_{i}(t, x) \geq 0$, for all $t, x$, and for all $i$, whereas (ii) yields boundedness: $v(t, x) \leq \rho$, for all $t, x$ with $\rho:=\max \left(\rho_{1}, \rho_{2}\right)+1$, thanks to the maximum principle.

As species all have the same dynamic in one-scale models, it is sufficient to consider a unique reactant, $i$. e. $M=1$. The function $R$ is written in the form $R(y)=b(y)-d(y)$, where $b$ and $d$ refer to variations due to births and deaths respectively. They are real valued polynomials with non-negative coefficients. We consider this case in this section.

We now consider an initial condition $v(0)=v_{0} \in C^{3}(I)$. In [24], Kotelenez showed that, under the preceding conditions, the Cauchy problem associated with (1) has a unique mild solution $v \in C\left(\mathbb{R}_{+} ; C^{3}(I)\right)$ satisfying

$$
v(t)=T(t) v_{0}+\int_{0}^{t} T(t-s) R(v(s)) d s,
$$

and $0 \leq v(t)<\rho$ for all $t \geq 0$. Here, $T(t):=\mathrm{e}^{\Delta t}$ is the semigroup associated with the Laplace operator $\Delta$ with periodic boundary conditions. We notice that $v_{0} \in C^{3}(I)$ is not necessary if the purpose is to solve the equation. Well-posedness still holds for much more general $v_{0}$. For more details, see [14], Section 5, or [3], Lemma 2 (based on [29]).

Informally, the (weak) infinitesimal generator of the PDE (1) reads

$$
\mathscr{A} \varphi(u)=\left(D_{u} \varphi(u), \Delta u+R(u)\right)=\left\langle D_{u} \varphi(u), \Delta u+R(u)\right\rangle_{2},
$$

for test functions $\varphi \in C_{b}^{1}\left(C^{1}(I)\right)$. The bracket $\langle\cdot, \cdot\rangle_{2}$ is the inner product of $L^{2}:=L^{2}(I)$, the space of square integrable real valued functions on $I$, and $(\cdot, \cdot)$ denotes the duality pairing. Let us give some probabilistic heuristics for understanding (2). Let $X(\cdot)$ be a solution to $(1)$, and denote by $\Phi(\cdot, u)$ the solution starting at $u \in$ $C^{3}(I)$. Viewing $(X(t))_{t \geq 0}$ as a $C^{3}(I)$-valued deterministic - thus Markov - process on some filtered probability space $\left(\Omega, \mathscr{F},\left(\mathscr{F}_{t}\right)_{t \geq 0}, \mathbb{P}\right)$, its semigroup $\left(P_{t}\right)_{t \geq 0}$ on $\mathscr{B}\left(C^{3}(I)\right)$ reads

$$
P_{t} \varphi(u):=\mathbb{E}[\varphi(X(t)) \mid X(0)=u]=\varphi(\Phi(t, u)) .
$$

Then as usual, we interpret the generator $\mathscr{A}$ as the derivative of the semigroup w.r.t. $t$, at $t=0$. Thus, for every test function $\varphi \in C_{b}^{1}\left(C^{1}(I)\right)$, the chain rule leads to

$$
\mathscr{A} \varphi(u):=\left.\frac{\partial}{\partial t} \varphi(\Phi(t, u))\right|_{t=0}=(D \varphi(u), \Delta u+R(u)) .
$$

Finally, $D \varphi(u)$ being the differential of $\varphi$ at $u$, it belongs to $\left(C^{1}(I)\right)^{\prime}$. We identify it with the (abstract) gradient of $\varphi$ at $u$ since $C^{1}(I) \subset L^{2}$, and write

$$
(D \varphi(u), \Delta u+R(u))=\langle D \varphi(u), \Delta u+R(u)\rangle_{2},
$$

thanks to the Riesz representation theorem. Relation (2) then follows.

Finally, we define the corresponding debit function as

$$
\psi(u)=\Delta u+R(u) .
$$

It is the vector field, in $C^{1}(I)$ here, determining the flow of the equation. More precisely, $u \mapsto \Delta u($ resp. $u \mapsto R(u))$ is the debit function related to diffusions (resp. reactions). 


\subsubsection{Stochastic spatial model}

Following [3], we divide the unit interval into $N$ smaller intervals of equal length $N^{-1}: I_{j}=\left((j-1) N^{-1}, j N^{-1}\right.$ ], for $j=1, \cdots, N$, called sites. Molecules are produced - birth - or removed - death - on each site according to chemical reactions. They also diffuse between sites by simple random walks, at rates proportional to $N^{2}$. This couples the site reactants and extends Kurtz's model (M3) to the spatially inhomogeneous case (M4). Define:

- $X_{j}^{N}$ : the number of molecules on site $j, j=1, \cdots, N$,

- $X^{N}=\left(X_{j}^{N}\right)_{1 \leq j \leq N} \in \mathbb{N}^{N}$ : the molecular composition of the whole system,

- $\mathfrak{R}$ : the set of possible onsite reactions (we assume that $\mathfrak{R}$ is finite).

Consider the natural completed filtration $\left\{\mathscr{F}_{t}^{N}, t \geq 0\right\}$, where $\mathscr{F}_{t}^{N}$ is the completion of the $\sigma$-algebra $\sigma\left(\left\{X^{N}(s): s \leq t\right\}\right)$ with null probability measure sets. This will be the filtration considered by default for each process we consider. From [30,31] or Chapter 4, Section 2, p.162-164 of [17], we know that $\left\{X^{N}(t), t \geq 0\right\}$ is a time homogeneous $\mathbb{N}^{N}$-valued jump Markov process, with the following transitions on any $j$ :

Onsite reactions. A chemical reaction $r \in \mathfrak{R}$ occurs on site $j$ :

$$
X_{j}^{N} \rightarrow X_{j}^{N}+\gamma_{j, r}, \quad \gamma_{j, r} \in \mathbb{Z}, \quad \text { at rate } \lambda_{r}\left(X_{j}^{N}\right) .
$$

Diffusions. A molecule moves from site $j$ to site $j-1$ or to site $j+1$ :

$$
\begin{cases}\left(X_{j-1}^{N}, X_{j}^{N}\right) \rightarrow\left(X_{j-1}^{N}+1, X_{j}^{N}-1\right), & \text { at rate } N^{2} X_{j}^{N}, \\ \left(X_{j}^{N}, X_{j+1}^{N}\right) \longrightarrow\left(X_{j}^{N}-1, X_{j+1}^{N}+1\right), & \text { at rate } N^{2} X_{j}^{N} .\end{cases}
$$

At the level of the whole vector, we get for $1 \leq j \leq N$

$$
\begin{cases}X^{N} \longrightarrow X^{N}+\gamma_{j, r} e_{j} & \text { at rate } \lambda_{r}\left(X_{j}^{N}\right), \\ X^{N} \longrightarrow X^{N}+e_{j-1}-e_{j} & \text { at rate } N^{2} X_{j}^{N} \\ X^{N} \longrightarrow X^{N}+e_{j+1}-e_{j} & \text { at rate } N^{2} X_{j}^{N}\end{cases}
$$

where $\left\{e_{j}, j=1, \cdots, N\right\}$ is the canonical basis of $\mathbb{R}^{N}$.

In all the article, we assume that the rates of onsite reactions are polynomial functions and that there are a finite number of reaction in $\mathfrak{R}$.

Two types of events are clearly distinguishable: onsite or chemical reactions, and diffusions. We sometimes say reaction for each of them, but we avoid any possibility of confusion. The vectors $\gamma_{j, r} e_{j}, e_{j-1}-e_{j}, \cdots$ appearing in transitions are the stocheometric coefficients of the corresponding event. They point out the different possible directions that the jump due to an event can take. We also denote them by jump height or simply jump.

We insist on the fact that the directions of jumps are actually characterized by two parameters: a "true" event - reaction or diffusion - and a location - site - where that event occurs. Furthermore, they are independently chosen and the total jump rate, denoted by $\lambda^{N}$, is the sum of the rates over all the possible directions of jump:

$$
\lambda^{N}\left(X^{N}\right)=\sum_{j=1}^{N}\left[\sum_{r \in \Re} \lambda_{r}\left(X_{j}^{N}\right)+N^{2}\left(X_{j-1}^{N}+2 X_{j}^{N}+X_{j+1}^{N}\right)\right] .
$$

In general, the jump height of an onsite reaction may depend both on the reaction and on the site where it occurs. In the present model, onsite reactions are assumed to be spatially homogeneous, that is

$$
\gamma_{j, r}=\gamma_{r} \text { for all } 1 \leq j \leq N \text {. }
$$

Well-posedness, generator and debit function. Such a Markov process can be constructed, relying on a sequence $\left(\tau_{i}\right)_{i \geq 1}$ of independent random waiting times with exponential distributions. We set $T_{0}=0$ and $T_{k}=\tau_{1}+\cdots+\tau_{k}$ for $k \geq 1$. $\left\{T_{k}, k \geq 1\right\}$ is a family of $\left\{\mathscr{F}_{t}^{N}\right\}$-stopping times such that $X^{N}$ is constant on each $\left[T_{k-1}, T_{k}\right)$, and has a jump at $T_{k}$. The parameter of $\tau_{k}$ is the total jump rate $\lambda^{N}\left(X^{N}\left(T_{k-1}\right)\right)$, at time $T_{k-1}$. For $t>0$,

$$
\mathbb{P}\left\{\tau_{k}>t \mid T_{k-1}\right\}=\exp \left\{-t \lambda^{N}\left(X^{N}\left(T_{k-1}\right)\right)\right\} .
$$

At time $T_{k}$, a given event (or direction of jump) is chosen with probability

$$
\frac{\text { rate of the direction of jump at time } \mathrm{T}_{k-1}}{\text { total rate of jump at time } \mathrm{T}_{k-1}} \text {. }
$$


Periodic boundary conditions are considered on the spatial domain, and $X_{j}^{N}$ is viewed as a sequence satisfying $X_{j+N}^{N}=X_{j}^{N}$ for all $j$ (as in [6], see p.10). The infinitesimal generator of our jump Markov process then reads

$$
\begin{aligned}
\mathscr{A}^{N} \varphi(X):= & \left.\frac{d}{d t} \mathbb{E}_{X}\left[\varphi\left(X^{N}(t)\right)\right]\right|_{t=0}:=\lim _{t \rightarrow 0} \frac{1}{t} \mathbb{E}_{X}\left[\varphi\left(X^{N}(t)\right)-\varphi(X)\right] \\
= & \sum_{j=1}^{N} \sum_{r \in \Re}\left[\varphi\left(X+\gamma_{r} e_{j}\right)-\varphi(X)\right] \lambda_{r}\left(X_{j}\right) \\
& +\sum_{j=1}^{N}\left[\varphi\left(X+e_{j-1}-e_{j}\right)+\varphi\left(X+e_{j+1}-e_{j}\right)-2 \varphi(X)\right] N^{2} X_{j} .
\end{aligned}
$$

We need not specify the complete domain of the generator $\mathscr{A}^{N}$. The limit above holds, for test functions $\varphi \in$ $C_{b}\left(\mathbb{R}^{N}\right)$ (see [17] p.162-164).

In this stochastic description, the global debit is the function which sums the jump heights weighted with the corresponding rates, under the possible directions of jump. It is a vector field in the state space. If we denote it by $\Psi^{N}$, then

$$
\begin{aligned}
\Psi^{N}(X): & =\sum_{j=1}^{N} \sum_{r \in \Re} \gamma_{r} \lambda_{r}\left(X_{j}\right) e_{j}+N^{2}\left(e_{j-1}-2 e_{j}+e_{j+1}\right) X_{j} \\
& =\sum_{j=1}^{N}\left(\sum_{r \in \Re} \gamma_{r} \lambda_{r}\left(X_{j}\right)+N^{2}\left(X_{j-1}-2 X_{j}+X_{j+1}\right)\right) e_{j}
\end{aligned}
$$

for $X \in \mathbb{N}^{N}$, thanks to periodicity and a change of subscript. We clearly identify the debit of onsite reactions, and that of diffusions. The latter, the linear part in $X$, defines a discrete Laplace operator that we introduce later.

Scaling and density dependence: The following are parts of the main assumptions in the framework of onescale models.

- On each site, the reactant has a population size of order $\mu$. Typically, $\mu$ is large and represents the initial average number of particles on each site.

- Density dependence holds for reaction rates. That is: for all $r \in \mathfrak{R}$, there exists $\tilde{\lambda}_{r}$ satisfying

$$
\lambda_{r}\left(X_{j}^{N}\right)=\mu \tilde{\lambda}_{r}\left(\frac{X_{j}^{N}}{\mu}\right) \quad \text { for all } j=1, \cdots, N
$$

Density dependence is a natural assumption when dealing with chemical reaction systems (see [30], or Chapter 11 of [17]). It clearly holds for linear rates for instance, and can be understood as the fact that reducing the scale is somehow increasing the density, and results in speeding up the dynamic.

In general $\mu$ depends on $N$ and we omit to mention the dependence on $\mu$.

We rescale our process, setting

$$
U_{j}^{N}:=U_{j}^{N, \mu}:=\frac{X_{j}^{N}}{\mu}, \quad \text { and } U^{N}:=\left(U_{j}^{N}\right)_{1 \leq j \leq N^{\prime}}
$$

and do not distinguish $\lambda_{r}$ from $\tilde{\lambda}_{r}$ in the rest of the article, for all $r \in \mathfrak{R}$. The new scaled process $U^{N}$ has values in $\mathbb{R}^{N}$ and gives the proportions of the reactant on each site. With some abuse, we often say concentration instead of proportion.

In order to achieve a pointwise modeling over the whole spatial domain, a space-time jump Markov process is constructed, which is the stochastic counterpart of the solution to (1). As in [3], [25] or [6, 7], we introduce the step function

$$
u^{N}(t, x)=\sum_{j=1}^{N} U_{j}^{N}(t) 1_{j}(x), \quad t \geq 0, \quad x \in I_{j},
$$

where $\mathbb{1}_{j}(\cdot):=\mathbb{1}_{I_{j}}(\cdot)$ is the indicator function of the $j$-th site $I_{j}$. Note that for all $t \geq 0, N \geq 1$, the function $u^{N}(t, \cdot)$ can be identified with the vector $U^{N}(t)$ of $\mathbb{R}^{N}$. Furthermore, since we consider a periodic framework we have $U_{j+N}^{N}(t)=U_{j}^{N}$ and $u^{N}(t, \cdot)$ is 1 - periodic. Below, we use the standard identification $u^{N}(t):=u^{N}(t, \cdot)$. 
Let $\mathbb{H}^{N}$ denote the subspace of $L^{2}$ which consists of real-valued and 1-periodic step functions that are constant on the intervals $I_{j}, 1 \leq j \leq N$. Therefore, $u^{N}:=\left\{u^{N}(t), t \geq 0\right\}$ is a càdlàg (time-space) Markov process, with values in $\mathbb{H}^{N}$ and jumps given by:

$$
\begin{cases}u^{N} \longrightarrow u^{N}+\frac{\gamma_{r}}{\mu} 1_{j}, & \text { at rate } \mu \lambda_{r}\left(u_{j}^{N}\right), \text { for } r \in \Re, \\ u^{N} \longrightarrow u^{N}+\frac{1_{j-1}-1_{j}}{\mu}, & \text { at rate } \mu N^{2} u_{j}^{N}, \text { for } 1 \leq j \leq N, \\ u^{N} \longrightarrow u^{N}+\frac{1_{j+1}-1_{j}}{\mu}, & \text { at rate } \mu N^{2} u_{j}^{N}, \text { for } 1 \leq j \leq N .\end{cases}
$$

Here, $u_{j}^{N}=U_{j}^{N}$ is the $j-$ th "coordinate" of $u^{N}$, obtained by the canonical projection

$$
\begin{aligned}
P_{N}: & L^{2} \rightarrow \mathbb{T}^{N} \\
& u \mapsto P_{N} u=\sum_{j=1}^{N} u_{j} 1_{j}, \quad \text { where } \quad u_{j}:=N \int_{I_{j}} u(x) d x .
\end{aligned}
$$

The infinitesimal generator $\mathscr{A}^{N}:=\mathscr{A}^{N, \mu}$ of $u^{N}$ is given by

$$
\begin{aligned}
\mathscr{A}^{N} \varphi(u) & =\sum_{j=1}^{N} \sum_{r \in \mathfrak{R}}\left[\varphi\left(u+\frac{\gamma_{r}}{\mu} 1_{j}\right)-\varphi(u)\right] \mu \lambda_{r}\left(u_{j}\right) \\
& +\sum_{j=1}^{N}\left[\varphi\left(u+\frac{1_{j-1}-1_{j}}{\mu}\right)+\varphi\left(u+\frac{1_{j+1}-1_{j}}{\mu}\right)-2 \varphi(u)\right] \mu N^{2} u_{j},
\end{aligned}
$$

on the domain $C_{b}\left(\mathbb{\boxplus}^{N}\right)$. It can be extended to $C_{b}\left(L^{2}\right)$ by

$$
\bar{A}^{N} \varphi(u):=\mathscr{A}^{N} \varphi\left(P_{N} u\right) .
$$

Such a Markov process does exist and is unique (see [28] whose result is based on [17]) until a possible blow-up time. In addition, under natural assumptions on reaction rates, we have $u^{N}(t) \geq 0$ for all $t$, as soon as $u^{N}(0) \geq 0$.

As already mentioned, the convergence of $u^{N}$ to $u$ solution of (1) has been the object of several articles. This implies in particular that for $N$ large enough there is no blow-up.

For $f, g \in \mathbb{T}^{N}$, the $L^{2}$ inner product reads $\langle f, g\rangle_{2}=N^{-1} \sum_{j=1}^{N} f_{j} g_{j}$. We denote by $\|\cdot\|_{2}$ the $L^{2}$ norm. Also, the supremum norm is given by $\|f\|_{\infty}=\sup _{1 \leq j \leq N}\left|f_{j}\right|$. Setting $\mathbb{W}:=\cup_{N \geq 1} \mathbb{\sharp}^{N}$, the following obviously holds.

\section{Proposition 2.1}

(i) $\left(\mathbb{Q}^{N},\langle\cdot, \cdot\rangle_{2}\right)$ is a finite dimensional Hilbert space with $\left\{\sqrt{N} \mathbb{1}_{j}, 1 \leq j \leq N\right\}$ as an orthonormal basis.

(ii) $\lim _{N \rightarrow \infty}\left\|P_{N} u-u\right\|_{2} \longrightarrow 0$ for $u \in L^{2}$, and $\left(\mathbb{H},\|\cdot\|_{2}\right)$ is dense in $\left(L^{2},\|\cdot\|_{2}\right)$.

(iii) $P_{N}$ is a contracting linear continuous operator on $\left(C_{p}(I),\|\cdot\|_{\infty}\right)$ such that $\mathbb{\uplus} \subset C_{p}(I)$ and $\lim _{N \rightarrow \infty}\left\|P_{N} u-u\right\|_{\infty} \longrightarrow 0$ for $u \in C_{p}(I)$.

\subsection{Multiscale stochastic spatial model}

Now we wish to generalize [12] to a spatially dependent context. We consider two orders of population sizes and use similar arguments as above to write down the infinitesimal generator. Note that at least two types of reactants are needed, i. e. $M \geq 2$ is necessary. For simplicity, we consider exactly two reactants, i. e. we take $M=2$, that we denote by $C$ and $D$. The former is abundant and the latter is rare. Henceforth, the super/subscript $C$ (resp. D) refers to the species $C$ (resp. D).

The set $\Re$ of possible onsite reactions is divided in three disjoint subsets

$$
\mathfrak{R}=\mathfrak{R}_{C} \cup \mathfrak{R}_{D C} \cup \mathfrak{R}_{D} .
$$

Reactions in $\mathfrak{R}_{C}$ (resp. $\mathfrak{R}_{D}$ ) involve only molecules of $C$ (resp. $D$ ) as reactants and products, whereas, reactions in $\Re_{D C}$ involve both types of reactants and/or products. We assume:

\section{Assumption 2.2}

(i) Density dependence holds for reactions in $\mathfrak{R}_{C}$.

(ii) Reactions $r \in \mathfrak{R}_{C}$ are spatially homogeneous and fast ("pure fast reactions"), whereas reactions $r \in \mathfrak{R}_{D}$ are slow ("pure slow reactions").

(iii) The molecules of $C$ diffuse, whereas those of $D$ do not. 
Now, we define

- $X_{j}^{N, C}\left(\right.$ resp. $\left.X_{j}^{N, D}\right)$ as the number of molecules of $C$ (resp. D) on site $j$,

- $X_{C}^{N}:=\left(X_{j}^{N, C}\right)_{1 \leq j \leq N^{\prime}} X_{D}^{N}:=\left(X_{j}^{N, D}\right)_{1 \leq j \leq N}$ and $X^{N}:=\left(X_{C}^{N}, X_{D}^{N}\right) \in \mathbb{N}^{2 N}$.

Scales are set such that on every site, the initial average number of molecules for $C$ is of order $\mu$, whereas it is of order $\kappa$ for $D$, with $\mu \gg \kappa$. Namely, if the total initial population is $\mathfrak{M}=\mathfrak{M}_{C}+\mathfrak{M}_{D}$, then $\mathfrak{M}_{C} \approx N \times \mu$ whereas $\mathfrak{M}_{D} \approx N \times \kappa$. We set

$$
\begin{aligned}
U_{j}^{N, C}:=U_{j}^{N, \mu, C}:=\frac{X_{j}^{N, C}}{\mu}, & U_{j}^{N, D}:=U_{j}^{N, \mu, D}:=X_{j}^{N, D} \\
U_{C}^{N}:=\left(U_{j}^{N, C}\right)_{1 \leq j \leq N^{\prime}} \quad & U_{D}^{N}:=\left(U_{j}^{N, D}\right)_{1 \leq j \leq N^{\prime}}
\end{aligned}
$$

and finally

$$
U^{N, \mu}=: U^{N}:=\left(U_{C}^{N}, U_{D}^{N}\right) .
$$

The new scaled process $U^{N}$ has values in $\mathbb{R}^{N} \times \mathbb{N}^{N}$. Its generator has the form

$$
\begin{aligned}
\mathscr{A}^{N, \mu} \varphi\left(U_{C}, U_{D}\right)= & \sum_{j=1}^{N}\left\{\sum_{r \in \Re_{C}}\left[\varphi\left(U_{C}+\frac{\gamma_{j, r}^{C}}{\mu} e_{j}, U_{D}\right)-\varphi\left(U_{C}, U_{D}\right)\right] \mu \lambda_{r}\left(U_{j}^{C}\right)\right. \\
& +\sum_{r \in \Re_{D C}}\left[\varphi\left(U_{C}+\frac{\gamma_{j, r}^{C}}{\mu} e_{j}, U_{D}+\gamma_{j, r}^{D} e_{j}\right)-\varphi\left(U_{C}, U_{D}\right)\right] \lambda_{r}\left(U_{j}^{C}, U_{j}^{D}\right) \\
& \left.+\sum_{r \in \Re_{D}}\left[\varphi\left(U_{C}, U_{D}+\gamma_{j, r}^{D} e_{j}\right)-\varphi\left(U_{C}, U_{D}\right)\right] \lambda_{r}\left(U_{j}^{D}\right)\right\} \\
& +\sum_{j=1}^{N}\left\{\left[\varphi\left(U_{C}+\frac{e_{j-1}-e_{j}}{\mu}, U_{D}\right)+\varphi\left(U_{C}+\frac{e_{j+1}-e_{j}}{\mu}, U_{D}\right)-2 \varphi\left(U_{C}, U_{D}\right)\right] \mu N^{2} U_{j}^{C}\right.
\end{aligned}
$$

for test functions $\varphi \in C_{b}\left(\mathbb{R}^{2 N}\right)$ and $U=\left(U_{C}, U_{D}\right) \in \mathbb{R}^{2 N}$.

Below, we discuss the nature of reactions in $\mathfrak{R}_{D C}$, and consider situations where $\gamma_{j}^{r, D}$ and $\lambda_{r}$ need to be rescaled, for $r \in \mathfrak{R}_{D}$ and some $r \in \mathfrak{R}_{D C}$.

Next, we introduce the step function

$$
u^{N}(t, x)=\sum_{j=1}^{N} U_{j}^{N}(t) \mathbb{1}_{j}(x), t \geq 0, x \in I_{j}
$$

which belongs to $\mathbb{t}^{N} \times \mathbb{H}^{N}$. It involves the following functions for each component $u_{C}^{N}(t, x)=$ $\sum_{j=1}^{N} u_{j}^{N, C}(t) \mathbb{1}_{j}(x)$ and $u_{D}^{N}(t, x)=\sum_{j=1}^{N} u_{j}^{N, D}(t) \mathbb{1}_{j}(x)$,

where $u_{j}^{N, C}(t):=P_{N} u_{C}^{N}(t)=N \int_{I_{j}} u_{C}^{N}(t, x) d x=U_{j}^{N, C}(t)$,

and a similar relation holds for the reactant $D$. Therefore, $u^{N}:=\left\{u^{N}(t), t \geq 0\right\}$ is a $\mathbb{G}^{N} \times \mathbb{G}^{N}$-valued càdlàg jump Markov process, with transitions:

$$
\begin{cases}\left(u_{C}^{N}, u_{D}^{N}\right) \rightarrow\left(u_{C}^{N}+\frac{\gamma_{j, r}^{C}}{\mu} \mathbb{1}_{j}, u_{D}^{N}\right), & \text { at rate } \mu \lambda_{r}\left(u_{j}^{N, C}\right), \text { for } r \in \Re_{C}, \\ \left(u_{C}^{N}, u_{D}^{N}\right) \rightarrow\left(u_{C}^{N}+\frac{\gamma_{j, r}^{C}}{\mu} \mathbb{1}_{j}, u_{D}^{N}+\gamma_{j, r}^{D} \mathbb{1}_{j}\right), & \text { at rate } \lambda_{r}\left(u_{j}^{N, C}, u_{j}^{N, D}\right), \text { for } r \in \Re_{D C}, \\ \left(u_{C}^{N}, u_{D}^{N}\right) \rightarrow\left(u_{C}^{N}, u_{D}^{N}+\gamma_{j, r}^{D} \mathbb{1}_{j}\right), & \text { at rate } \lambda_{r}\left(u_{j}^{N, D}\right), \text { for } r \in \mathfrak{R}_{D^{\prime}} \\ \left(u_{C}^{N}, u_{D}^{N}\right) \rightarrow\left(u_{C}^{N}+\frac{\mathbb{1}_{j-1}-\mathbb{1}_{j}}{\mu}, u_{D}^{N}\right), & \text { at rate } N^{2} u_{j}^{N, C}, \text { for } 1 \leq j \leq N, \\ \left(u_{C}^{N}, u_{D}^{N}\right) \rightarrow\left(u_{C}^{N}+\frac{\mathbb{1}_{j+1}-\mathbb{1}_{j}}{\mu}, u_{D}^{N}\right), & \text { at rate } N^{2} u_{j}^{N, C}, \text { for } 1 \leq j \leq N .\end{cases}
$$

We endow $\mathbb{t}^{N} \times \mathbb{\sharp}^{N}$ with the norm

$$
\left\|\left(f_{1}, f_{2}\right)\right\|_{\infty, \infty}:=\left\|f_{1}\right\|_{\infty}+\left\|f_{2}\right\|_{\infty}, \text { for } f_{1}, f_{2} \in \mathbb{H}^{N} .
$$


As before, we assume that onsite reactions in $\Re_{C}$ are spatially homogeneous, that is

$$
\gamma_{j, r}^{C}=\gamma_{r}^{C} \text { for all } 1 \leq j \leq N, r \in \mathfrak{R}_{C} .
$$

Let us specify the description of the mixed reactions.

\section{Assumption 2.3}

In some $S_{1} \subset \mathfrak{R}_{D C}$, reactions are spatially homogeneous, fast and do not affect the discrete species:

- $\gamma_{j, r}^{D}=0$ and the rate is $\mu \tilde{\lambda}_{r}\left(u_{j}^{N, C}, u_{j}^{N, D}\right)$ for $r \in S_{1} \subset \mathfrak{R}_{D C}$.

Again we omit the tilde below.

The limit $N \rightarrow \infty$ of the above system creates mathematical difficulties (see Remark 3.3 and Remark A.1 below). We introduce some spatial correlations for reactions in $\left(\mathfrak{R}_{D C} \backslash S_{1}\right) \cup \mathfrak{R}_{D}$.

All reactions in $\left(\Re_{D C} \backslash S_{1}\right) \cup \Re_{D}$ are slow. We assume that when such a reaction occurs on a site, it affects the neighboring sites. A natural way to do this is to assume that when reaction $r \in\left(\Re_{D C} \backslash S_{1}\right) \cup \mathfrak{R}_{D}$ occurs at a site $j \in\{1, \ldots, N\}$, each site $i \in\{1, \ldots, N\}$, is affected in the following way:

$$
\left(u_{j}^{N, C}, u_{j}^{N, D}\right) \longrightarrow\left(u_{j}^{N, C}+\frac{\gamma_{r}^{C}}{\mu} \gamma_{i j}^{N}, u_{j}^{N, D}+\gamma_{r}^{D} \gamma_{i j}^{N}\right) .
$$

A first possibility is to take $\gamma_{i j}^{N}=\frac{1}{A}$ for $|i-j|<A$, describing the case when a reaction at site $i$ triggers simultaneously the same reaction at sites at distance less than $\frac{A}{N}$, while keeping the global effect of order 1 . The above model corresponds to $A=1$ but below we assume that $A$ has the same order as $N$. More generally, we choose a 1-periodic $C_{p}(I)$ function $a$ and choose:

$$
\gamma_{i j}^{N}=\int_{I_{i}} a\left(x-\frac{j}{N}\right) d x
$$

It is natural to assume that $a$ is maximum at zero, and is even decaying w.r.t. $|x|$. The preceding case is recovered with $a$ being an indicator function. Note that we should require $\gamma_{r}^{D} \gamma_{i j}^{N}$ to be an integer, but it is complicated to write general conditions to ensure this.

This modeling has a problem. Indeed, the positivity of the proportion of molecules is ensured by the fact that the rate vanishes when the proportion vanishes, but now, since we have a nonlocal effect, this is not true. It would not be realistic to consider a rate which depends on the proportion at all neighboring sites. Instead, we simply assume that when a reaction occurs at site $j$, it has an effect at a site $i$ only if that reaction is possible. Thus we would like to replace (10) by

$$
\left(u_{j}^{N, C}, u_{j}^{N, D}\right) \rightarrow\left(u_{j}^{N, C}+\frac{\gamma_{r}^{C}}{\mu} \gamma_{i j}^{N} \theta_{i j}^{r}, u_{j}^{N, D}+\gamma_{r}^{D} \gamma_{i j}^{N} \theta_{i j}^{r}\right)
$$

with $\theta_{i j}^{r}=\theta_{i j}^{r}\left(u_{j}^{N, C}, u_{j}^{N, D}\right)=\mathbb{1}_{u_{j}^{N, C}+\frac{\gamma_{r}^{C}}{\mu} \gamma_{i j}^{N} \geq 0} \mathbb{1}_{u_{j}^{N, D}+\gamma_{r}^{D} \gamma_{i j}^{N} \geq 0}$, where $\gamma_{r}^{C}=0$ for $r \in \mathfrak{R}_{D}$. Again, this creates mathematical difficulties, since the indicator function is not smooth. We replace it by a smooth function $\theta$ equal to 0 on $(-\infty, 0]$, to 1 on $[1, \infty)$ and smoothly increasing on $[0,1]$. We are now able to write the multiscale model we consider.

\section{Assumption 2.4} way:

When a slow reaction $r \in\left(\Re_{D C} \backslash S_{1}\right) \cup \Re_{D}$ occurs on a site $j$, it affects $C$ and $D$ on a site $1 \leq i \leq N$ in the following

$$
\gamma_{j, r}^{C} \equiv \gamma_{j, r}^{N, C}\left(u_{C}^{N}, u_{D}^{N}\right):=\gamma_{r}^{C} \sum_{i=1}^{N} \gamma_{i j}^{N} \mathbb{1}_{i} \theta_{i j}^{r}\left(u_{i}^{N, C}, u_{i}^{N, D}\right)
$$

and

$$
\gamma_{j, r}^{D} \equiv \gamma_{j, r}^{N, D}\left(u_{C}^{N}, u_{D}^{N}\right):=\gamma_{r}^{D} \sum_{i=1}^{N} \gamma_{i j}^{N} \rrbracket_{i} \theta_{i j}^{r}\left(u_{i}^{N, C}, u_{i}^{N, D}\right),
$$

where $\gamma_{i j}^{N}:=\int_{I_{i}} a\left(x-\frac{j}{N}\right) d x$, and $\theta_{i j}^{r}\left(u_{i}^{N, C}, u_{i}^{N, D}\right)=\theta\left(u_{i}^{N, C}+\frac{\gamma_{r}^{C}}{\mu} \gamma_{i j}^{N}\right) \theta\left(u_{i}^{N, D}+\gamma_{r}^{D} \gamma_{i j}^{N}\right)$. 


\section{Remark 2.1}

Assumption 2.4 reflects some spatial correlation for slow reactions. The slow reactions start on a source site $j$, and then, their effect spreads inside the system. This is a natural assumption in molecular biology. In the cell, the synthesis of some rare proteins activates a sequence of chemical reactions in chain. This kind of phenomenon precedes the activation of a gene on DNA (DeoxyriboNucleic Acid) for instance.

Since $\mathbb{1}_{i}(x)$ is nonzero only on $I_{i}$ and $\gamma_{i j}^{N} \leq \frac{a(0)}{N}$, we clearly have:

$$
\left\|\gamma_{j, r}^{N, C}\left(u_{C}^{N}, u_{D}^{N}\right)\right\|_{\infty} \leq \frac{\left|\gamma_{r}^{C}\right| a(0)}{N} \text { and }\left\|\gamma_{j, r}^{N, D}\left(u_{C}^{N}, u_{D}^{N}\right)\right\|_{\infty} \leq \frac{\left|\gamma_{r}^{D}\right| a(0)}{N} \text {. }
$$

The infinitesimal generator of $u^{N}$ on $C_{b}\left(\mathbb{q}^{N} \times \mathbb{\boxplus}^{N}\right)$ then reads

$$
\begin{aligned}
\mathscr{A}^{N} \varphi\left(u_{C}, u_{D}\right)= & \sum_{j=1}^{N}\left\{\sum_{r \in \Re_{C}}\left[\varphi\left(u_{C}+\frac{\gamma_{r}^{C}}{\mu} \mathbb{1}_{j}, u_{D}\right)-\varphi\left(u_{C}, u_{D}\right)\right] \mu \lambda_{r}\left(u_{j}^{C}\right)\right. \\
& +\sum_{r \in S_{1}}\left[\varphi\left(u_{C}+\frac{\gamma_{r}^{C}}{\mu} \mathbb{1}_{j}, u_{D}\right)-\varphi\left(u_{C}, u_{D}\right)\right] \mu \lambda_{r}\left(u_{j}^{C}, u_{j}^{D}\right) \\
& +\sum_{r \in \Re_{D C} \backslash S_{1}}\left[\varphi\left(u_{C}+\frac{\gamma_{j, r}^{N, C}\left(u_{C}, u_{D}\right)}{\mu}, u_{D}+\gamma_{j, r}^{N, D}\left(u_{C}, u_{D}\right)\right)-\varphi\left(u_{C}, u_{D}\right)\right] \times \lambda_{r}\left(u_{j}^{C}, u_{j}^{D}\right) \\
& \left.+\sum_{r \in \Re_{D}}\left[\varphi\left(u_{C}, u_{D}+\gamma_{j, r}^{N, D}\left(u_{C}, u_{D}\right)\right)-\varphi\left(u_{C}, u_{D}\right)\right] \lambda_{r}\left(u_{j}^{D}\right)\right\} \\
& +\sum_{j=1}^{N}\left[\varphi\left(u_{C}+\frac{\mathbb{1}_{j-1}-\mathbb{1}_{j}}{\mu}, u_{D}\right)+\varphi\left(u_{C}+\frac{\mathbb{1}_{j+1}-\mathbb{1}_{j}}{\mu}, u_{D}\right)-2 \varphi\left(u_{C}, u_{D}\right)\right] \mu N^{2} u_{j}^{C} .
\end{aligned}
$$

It can be extended to $C_{b}\left(L^{2} \times L^{2}\right)$ by

$$
\mathscr{A}^{N} \varphi\left(u_{C}, u_{D}\right):=\mathscr{A}^{N, \mu} \varphi\left(P_{N} u_{C}, P_{N} u_{D}\right) .
$$

For $u:=\left(u_{C}, u_{D}\right) \in L^{2} \times L^{2}$, we often use the notation $\tilde{P}_{N} u:=\left(P_{N} u_{C}, P_{N} u_{D}\right)$.

Again, such a process exists until a possible blow-up time. If the process blows up, we say that from this time it takes a cemetery value $\bar{\Delta}$ whose distance to any point is 1 .

\subsection{Main result: convergence of (M6)}

We discuss the asymptotic behavior as $N$ and $\mu$ go to infinity of the sequence $u^{N}$ of Markov processes representing (M6) and described in the previous section for polynomial (onsite) reaction rates. More precisely, we prove that $u^{N}$ converges to $u$ solution of the coupled equations:

$$
\frac{\partial v_{C}}{\partial t}=\Delta v_{C}+F\left(v_{C}, v_{D}\right)(\mathrm{PDE}) \text { coupled to (ODE) } \frac{d v_{D}}{d t}=G\left(v_{C}, v_{D}\right) .
$$

where $G(u)=\theta\left(u_{C}\right) \theta\left(u_{D}\right) a * g(u), F$ and $g$ are the debit functions associated to the continuous and discrete species, see (17),(19) and (20) below. The precise result is given in Theorem 2.2.

This result is a law of large numbers. It differs from the classical one proved first in [3]. This is essentially due to the additional presence of discrete species in our model, which leads to multiscaling. That consideration complements classical one-scale stochastic spatial models, which were already very useful, to more realistic situation.

With the results in [12] in mind in the homogeneous case, it may seem counter-intuitive that stochasticity disappears at the limit. This is due to the fact that in our setting the discrete species are still in large number - even though the continuous ones are much more abundant. The number of discrete molecules is of order $N$ while the number of continuous ones is of order $\mu N$ and $\mu \rightarrow \infty$ faster than $\log N$.

Thus even if the initial model (M6) is a typical hybrid stochastic system, its limit may have no discrete variable anymore, and jumps totally disappear. Our result gives conditions under which one does not obtain a hybrid approximation for a multiscale spatial model. That is opposite to many recent common hybrid modeling and algorithimic approaches, where a hybrid stochastic behavior is considered for similar biological systems 
$[19,20,34,39,40]$. In a forthcoming work, we study another situations where the discrete species have a different scale also in space, hence their number is not large, stochasticity remains in the limit and an infinite dimensional PDMP appears, as an offspring of reaction-diffusion PDEs and jump processes.

We believe that our rigorous approach allows to state precise conditions which can be used to simplify such stochastic models with spatial dependence.

Our results can be extended to higher dimensions at the level of both the space and the number of species, at the prize of very cumbersome notation. The extension to a larger number of species is more of less trivial. For higher dimension in space, one has to be careful about particular details including for instance the resolution of the partial differential equations.

In the near future, we will study the fluctuations of the model around its deterministic limit, and get the speeds of convergence of the initial fast and slow dynamics through a central limit theorem.

Our present and future results will pave the way to making statistical inference, using inverse problem methods. Based on empirical data, it would be interesting to estimate the parameters involved in the model (pointwise and with confidence regions) and to try and validate the model. Estimating procedures may also, in time, prove useful to elaborate control strategies. Numerous inverse problem methods are available for deterministic models (see for instance [11,35, 36, 42] and references therein), but not so many for stochastic models in the presence of limited data. A law of large numbers, as established in our work, and our future central limit theorem could be instrumental in the development of such a method. A law of large numbers, as established in our work, provides a tool for this.

In the remaining of this section we first take formally the limit of the generator and obtain the generator of the coupled system above. Then we introduce some notations and the limit equations so that we can state rigorously our main result.

\subsubsection{Identification of the limit}

Some preliminaries: a discrete Laplace. For $f \in C_{p}(I)$ and for $x \in[0,1]$, we set

$$
\nabla_{N}^{+} f(x):=N\left[f\left(x+\frac{1}{N}\right)-f(x)\right] \text { and } \nabla_{N}^{-} f(x):=N\left[f(x)-f\left(x-\frac{1}{N}\right)\right] .
$$

Then, we define the discrete Laplace

$$
\begin{aligned}
\Delta_{N} f(x) & :=\nabla_{N}^{+} \nabla_{N}^{-} f(x) \\
& =\nabla_{N}^{-} \nabla_{N}^{+} f(x)=N^{2} f\left[\left(x-\frac{1}{N}\right)-2 f(x)+f\left(x+\frac{1}{N}\right)\right] .
\end{aligned}
$$

If $f \in \mathbb{M}^{N}$ in particular, then

$$
\Delta_{N} f(x)=\sum_{j=1}^{N}\left[N^{2}\left(f_{j-1}-2 f_{j}+f_{j+1}\right)\right] \mathbb{1}_{j}(x) .
$$

From the spectral analysis of $\Delta_{N}$, it is well known that, if $N$ is an odd integer, letting $0 \leq m \leq N-1$ with $m$ even, letting $\varphi_{0, N} \equiv 1, \varphi_{m, N}(x)=\sqrt{2} \cos \left(\pi m j N^{-1}\right)$ and $\psi_{m, N}(x)=\sqrt{2} \sin \left(\pi m j N^{-1}\right)$ for $x \in I_{j}$, then, $\left\{\varphi_{m, N}, \psi_{m, N}\right\}$ are eigenfunctions of $\Delta_{N}$ with eigenvalues given by $-\beta_{m, N}=-2 N^{2}\left(1-\cos \left(\pi m N^{-1}\right)\right) \leq 0$. If $N$ is even, we need the additional eigenfunction $\varphi_{N, N}=\cos (\pi j)$ for $x \in I_{j}$. The following (classical) properties are derived from [6], Lemma 2.12 p.12, [7], Lemma 4.2 for the parts (i)-(vi), and from [23], Chapter 9, Section 3 for the part (vi).

\section{Proposition 2.2}

(Some properties of the discrete Laplace)

(i) The family $\left\{\varphi_{m, N}, \psi_{m, N}\right\}$ forms an orthonormal basis of $\left(\mathbb{Q}^{N},\langle\cdot, \cdot\rangle_{2}\right)$.

Consider $f, g \in \mathbb{\square}^{N}$ and let $T_{N}(t)=e^{\Delta_{N} t}$ denote the semigroup on $\mathbb{\boxplus}^{N}$ generated by $\Delta_{N}$.

(ii) $T_{N}(t) f=\sum_{m} \mathrm{e}^{-\beta_{m, N} t}\left(\left\langle f, \varphi_{m, N}\right\rangle_{2} \varphi_{m, N}+\left\langle f, \psi_{m, N}\right\rangle_{2} \psi_{m, N}\right)$.

(iii) $\left\langle\nabla_{N}^{+} f, g\right\rangle_{2}=\left\langle f, \nabla_{N}^{-} g\right\rangle_{2}$ and $T_{N}(t) \Delta_{N} f=\Delta_{N} T_{N}(t) f$.

(iv) $\Delta_{N}$ and $T_{N}(t)$ are self-adjoint on $\left(\mathbb{Q}^{N},\langle\cdot, \cdot\rangle_{2}\right)$.

(v) $T_{N}(t)$ is a positive contraction semigroup on both $\left(\mathbb{\boxplus}^{N},\langle\cdot, \cdot\rangle_{2}\right)$ and $\left(\mathbb{\boxplus}^{N},\|\cdot\|_{\infty}\right)$. 
(vi) The projection $P_{N}$ commutes with $\Delta_{N}$, and for all $T>0, f \in C^{3}(I)$,

$$
\left\{\begin{array}{l}
\left\|\Delta_{N} f-\Delta f\right\| \infty \rightarrow 0 \text { as } N \rightarrow \infty, \\
\left\|\Delta_{N} P_{N} f-\Delta f\right\|_{\infty} 0 \rightarrow \text { as } N \rightarrow \infty, \\
\sup _{t \in[0, T]}\left\|T_{N}(t) P_{N} f-T(t) f\right\| \infty \rightarrow 0 s N \rightarrow \infty .
\end{array}\right.
$$

We introduce the operator $\tilde{\Delta}_{N}:=\left(\begin{array}{cc}\Delta_{N} & 0 \\ 0 & 0\end{array}\right)$ on $\mathbb{\boxplus}^{N} \times \mathbb{\boxplus}^{N}$, and denote by $\tilde{T}_{N}(t):=\mathrm{e}^{\tilde{\Delta}_{N} t}$ the associated semigroup.

(vii) $\tilde{\Delta}_{N}$ is a bounded linear operator on $\left(\mathbb{M}^{N} \times \mathbb{M}^{N},\|\cdot\|_{\infty, \infty}\right)$.

(viii) $\tilde{T}_{N}(t)=\left(\begin{array}{cc}T_{N}(t) & 0 \\ 0 & I_{d}\end{array}\right)$, and defines a bounded positive contraction semigroup on $\left(\mathbb{M}^{N} \times \mathbb{M}^{N},\|\cdot\|_{\infty, \infty}\right)$. We have denoted by $I_{d}$ is the identity operator on $\mathbb{\boxplus}^{N}$.

Debit functions and formal limit of the generator. We consider the generator $\mathscr{A}^{N}$ of (M6) given by (13), applied to a $C_{b}^{2}\left(\mathbb{G}^{N} \times \mathbb{M}^{N}\right)$ test function $\varphi$. We use a Taylor expansion of order 2 of $\varphi$. From Section 2.1.2, we know that the generator is (informally) obtained as the image of the corresponding debit by the differential of the test function.

In Appendix A.1, we give heuristics showing that the terms related to the second order of the Taylor expansion vanish, under the very strong condition $\mu^{-1} N^{2} \rightarrow 0$ as $N, \mu \rightarrow \infty$. In our rigorous proof below, we only assume $\mu^{-1} \log N \rightarrow 0$.

Concerning the first order terms, we identify the differential operator with the gradient as usual, and get

$$
\begin{aligned}
\left(D \varphi\left(u_{C}, u_{D}\right), \Psi^{N}\left(u_{C}, u_{D}\right)\right)= & \left\langle D^{1,0} \varphi\left(u_{C}, u_{D}\right), \sum_{j=1}^{N}\left(\sum_{r \in \mathfrak{R}_{C}} \gamma_{r}^{C} \lambda_{r}\left(u_{j}^{C}\right)+\sum_{r \in S_{1}} \gamma_{r}^{C} \lambda_{r}\left(u_{j}^{C}, u_{j}^{D}\right)\right) \mathbb{1}_{j}\right\rangle_{2} \\
& +\left\langle D^{1,0} \varphi\left(u_{C}, u_{D}\right), \sum_{j=1}^{N}\left(\sum_{r \in \mathfrak{R}_{D C} \backslash S_{1}} \frac{\gamma_{j, r}^{N, C}\left(u_{C}, u_{D}\right)}{\mu} \lambda_{r}\left(u_{j}^{C}, u_{j}^{D}\right)\right) \mathbb{1}_{j}\right\rangle_{2} \\
& +\left\langle D^{1,0} \varphi\left(u_{C}, u_{D}\right), \sum_{j=1}^{N} N^{2}\left(\mathbb{1}_{j-1}-2 \mathbb{1}_{j}+\mathbb{1}_{j+1}\right) u_{j}^{C}\right\rangle_{2} \\
& +\left\langle D^{0,1} \varphi\left(u_{C}, u_{D}\right), \sum_{j=1}^{N}\left(\sum_{r \in \mathfrak{R}_{D C} \backslash S_{1}} \gamma_{j, r}^{N, D}\left(u_{C}, u_{D}\right) \lambda_{r}\left(u_{j}^{C}, u_{j}^{D}\right)+\sum_{r \in \mathfrak{R}_{D}} \gamma_{j, r}^{N, D}\left(u_{C}, u_{D}\right) \lambda_{r}\left(u_{j}^{D}\right)\right)\right\rangle_{2}
\end{aligned}
$$

where $D^{1,0}$ (resp. $\left.D^{0,1}\right)$ denotes the differential w.r.t. $u_{C}\left(\right.$ resp. $\left.u_{D}\right)$. by

$$
\Psi_{C}^{N}\left(u_{C}, u_{D}\right):=\Delta_{N} u_{C}+F\left(u_{C}, u_{D}\right)+F_{1}^{N}\left(u_{C}, u_{D}\right)
$$

The first term on the right hand side (r.h.s.) is the debit of the diffusions of molecules of C. As in (4), it is obtained using the periodicity and a change of index:

$$
\sum_{j=1}^{N} N^{2}\left(\mathbb{1}_{j-1}-2 \mathbb{1}_{j}+\mathbb{1}_{j+1}\right) u_{j}^{C}=\sum_{j=1}^{N} N^{2}\left(u_{j+1}^{C}-2 u_{j}^{C}+u_{j-1}^{C}\right) \mathbb{1}_{j}=\Delta_{N} u_{C} .
$$

The second term is associated with fast onsite reactions (they all influence $C$ ). It is given by

$$
\begin{aligned}
F\left(u_{C}, u_{D}\right) & =\sum_{j=1}^{N}\left(\sum_{r \in \Re_{C}} \gamma_{r}^{C} \lambda_{r}\left(u_{j}^{C}\right)+\sum_{r \in S_{1}} \gamma_{r}^{C} \lambda_{r}\left(u_{j}^{C}, u_{j}^{D}\right)\right) \mathbb{1}_{j} \\
& =\sum_{r \in \Re_{C}} \gamma_{r}^{C} \lambda_{r}\left(u_{C}\right)+\sum_{r \in S_{1}} \gamma_{r}^{C} \lambda_{r}\left(u_{C}, u_{D}\right) .
\end{aligned}
$$

The third term corresponds to slow onsite reactions influencing $C$ and vanishes at the limit. It is defined by

$$
F_{1}^{N}\left(u_{C}, u_{D}\right)=\frac{1}{\mu} \sum_{j=1}^{N} \sum_{r \in \Re_{D C} \backslash S_{1}} \gamma_{r}^{C} \sum_{i=1}^{N} \gamma_{i j}^{N} \mathbb{1}_{i} \theta_{i j}^{r}\left(u_{i}^{C}, u_{i}^{D}\right) \lambda_{r}\left(u_{j}^{C}, u_{j}^{D}\right) .
$$


On the other hand, we have $\Psi_{D}^{N}: \mathbb{\square}^{N} \times \mathbb{\square}^{N} \rightarrow \mathbb{\square}^{N}$, the debit related to $D$. It corresponds to slow onsite reactions - only them has an effect on $D$ - and is given by

$$
\Psi_{D}^{N}\left(u_{C}, u_{D}\right)=\sum_{j=1}^{N} \sum_{i=1}^{N} \gamma_{i j}^{N} 1_{i} g_{i j}\left(u_{C}, u_{D}\right)=: G^{N}\left(u_{C}, u_{D}\right),
$$

where

$$
g_{i j}\left(u_{C}, u_{D}\right)=\sum_{r \in \Re_{D C} \backslash S_{1}} \gamma_{r}^{D} \theta_{i j}^{r}\left(u_{i}^{C}, u_{i}^{D}\right) \lambda_{r}\left(u_{j}^{C}, u_{j}^{D}\right)+\sum_{r \in \Re_{D}} \gamma_{r}^{D} \theta_{i j}^{r}\left(u_{i}^{C}, u_{i}^{D}\right) \lambda_{r}\left(u_{j}^{D}\right) .
$$

The global debit on $\mathbb{\boxplus}^{N} \times \mathbb{\boxplus}^{N}$ then reads

$$
\Psi^{N}\left(u_{C}, u_{D}\right)=\left(\Psi_{C}^{N}\left(u_{C}, u_{D}\right), \Psi_{D}^{N}\left(u_{C}, u_{D}\right)\right) .
$$

We finally define the extra debit type function $G: C_{p}(I) \times C_{p}(I) \rightarrow C_{p}(I)$, thanks to

$$
G\left(u_{C}, u_{D}\right)(x):=\theta\left(u_{C}(x)\right) \theta\left(u_{D}(x)\right) \int_{0}^{1} g\left(u_{C}, u_{D}\right)(y) a(x-y) d y
$$

for all $x \in I$. It is the limit of $G^{N}$ (see appendix A.4). Here,

$$
g\left(u_{C}, u_{D}\right)=\sum_{r \in \Re_{D C} \backslash S_{1}} \gamma_{r}^{D} \lambda_{r}\left(u_{C}, u_{D}\right)+\sum_{r \in \Re_{D}} \gamma_{r}^{D} \lambda_{r}\left(u_{D}\right)
$$

\section{Remark 2.2}

We notice that $\Delta_{N}, F, F_{1}^{N}$ and $G^{N}$ map $C_{p}(I) \times C_{p}(I)$ on $C_{p}(I)$. This follows from the definition of the discrete Laplace, and from we are considering polynomial reaction rates. Here above, we have considered these three functions on $\mathbb{t}^{N} \times \mathbb{t}^{N}$, as they were introduced to define the debit function, on $\mathbb{t}^{N} \times \mathbb{t}^{N}$.

Besides, the debit can be extended to $C_{p}(I) \times C_{p}(I)$, not by taking $\Delta_{N}, F, F_{1}^{N}$ and $G^{N}$ as maps on $C_{p}(I) \times C_{p}(I)$, but using the projection $P_{N}$ introduced earlier, in a similar way as for extending the generator. Namely, the global debit extends to

$$
\left(u_{C}, u_{D}\right) \mapsto\left(\Psi_{C}^{N}\left(P_{N} u_{C}, P_{N} u_{D}\right), \Psi_{D}^{N}\left(P_{N} u_{C}, P_{N} u_{D}\right)\right) .
$$

Furthermore, it should be emphasized that each of $F, F_{1}^{N}$ and $\lambda_{r}, r \in \mathfrak{R}$ is a function of real variables, actually. thanks to

$$
f(u)(x)=F(u(x)), x \in[0,1] .
$$

If $F$ is (locally) Lipschitz, then $f$ is (locally) Lipschitz too, accordingly, with the corresponding norms. We do not distinguish between $F$ and $f$ in our notation, and do the same for the functions $F_{1}^{N}$ and $\lambda_{r}, r \in \Re$.

Fix $u=\left(u_{C}, u_{D}\right) \in C_{p}(I) \times C_{p}(I)$ and let $N, \mu \rightarrow \infty$. Clearly, $F_{1}^{N}\left(\tilde{P}_{N} u\right) \rightarrow 0$. From Proposition 2.1 and the continuity of $F, F\left(\tilde{P}_{N} u\right) \rightarrow F(u)$. Therefore, if $u_{C} \in C^{3}(I), \Psi_{C}^{N}\left(\tilde{P}_{N} u\right) \rightarrow \Delta u_{C}+F(u)$ thanks to Proposition 2.2 (vi). We also prove below that $\Psi_{D}^{N}\left(\tilde{P}_{N} u\right)=G^{N}\left(\tilde{P}_{N} u\right) \rightarrow G(u)$. Therefore, under the strong assumption $\mu^{-1} N^{2} \rightarrow 0$ and $u_{C} \in C^{3}(I)$, we obtain the limit generator

$$
\begin{aligned}
\mathscr{A}^{\infty} \varphi\left(u_{C}, u_{D}\right)= & \left\langle D^{1,0} \varphi\left(u_{C}, u_{D}\right), \Delta u_{C}+F\left(u_{C}, u_{D}\right)\right\rangle_{2} \\
& +\left\langle D^{0,1} \varphi\left(u_{C}, u_{D}\right), G\left(u_{C}, u_{D}\right)\right\rangle_{2} .
\end{aligned}
$$

The condition $\mu^{-1} N^{2} \rightarrow 0$ was used By Arnold and Theodosopulu in [3], to prove a LLN for (M4) in the $L^{2}$ norm. However, that condition is not optimal. Indeed, in [7], Blount proved a LLN for (M4) in the supremum norm, requiring only $\mu^{-1} \log N \rightarrow 0$. Our convergence result for (M6) falls within the latter framework.

We now look for a process admitting $\mathscr{A}^{\infty}$ defined by (21) as infinitesimal generator. 


\subsubsection{The limiting problem}

Well-posedness. Proceding as in Section 2.1.1, we see that to $\mathscr{A}^{\infty}$, corresponds

$$
\frac{\partial v_{C}}{\partial t}=\Delta v_{C}+F\left(v_{C}, v_{D}\right)(\mathrm{PDE}) \text { coupled to (ODE) } \frac{d v_{D}}{d t}=G\left(v_{C}, v_{D}\right)
$$

Hence, we shall consider the following system of differential equations, for $x \in I, t \geq 0$, with periodic boundary conditions and initial data $v_{0}=\left(v_{0}^{C}, v_{0}^{D}\right)$ :

$$
\left\{\begin{array}{l}
\frac{\partial}{\partial t} v_{C}(t, x)=\Delta v_{C}(t, x)+F\left(v_{C}(t, x), v_{D}(t, x)\right) \\
\frac{\partial}{\partial t} v_{D}(t, x)=G\left(v_{C}(t, x), v_{D}(t, x)\right) \\
v_{C}(t, 0)=v_{C}(t, 1) \text { and } v_{D}(t, 0)=v_{D}(t, 1), t \geq 0 \\
v_{C}(0, x)=v_{0}^{C}(x) \geq 0 \text { and } v_{D}(0, x)=v_{0}^{D}(x) \geq 0 .
\end{array}\right.
$$

A compact form of the system is:

$$
\left\{\begin{array}{l}
\frac{d}{d t} v(t)=\tilde{\Delta} v(t)+R(v(t)) \\
v(t, 0)=v(t, 1), t \geq 0 \\
v(0)=v_{0} \geq 0
\end{array}\right.
$$

where $\tilde{\Delta}=\left(\begin{array}{cc}\Delta & 0 \\ 0 & 0\end{array}\right): C_{p}(I) \times C_{p}(I) \rightarrow C_{p}(I)$ is a linear operator we define on the domain $C^{2}(I) \times C_{p}(I)$, and whose associated semigroup $\tilde{T}(t):=\mathrm{e}^{\tilde{\Delta} t}$ is of contraction. For $u \in C_{p}(I) \times C_{p}(I), R(u)=\left(\begin{array}{l}F(u) \\ G(u)\end{array}\right)$, with $G(u)=\theta\left(u_{C}\right) \theta\left(u_{D}\right) a * g(u)$.

Before, we give the counterpart of Assumption 2.1 for (22), let us introduce the $\mathbb{H}^{N}$-valued function $|g|$ defined by $|g|(u):=\sum_{j=1}^{N}|g|_{j}(u) \mathbb{1}_{j}$ for $u=\left(u_{C}, u_{D}\right) \in C_{p}(I) \times C_{p}(I)$, where

$$
|g|_{j}\left(u_{C}, u_{D}\right):=\sum_{r \in \mathscr{R}_{D C} \backslash S_{1}}\left|\gamma_{r}^{D}\right| \lambda_{r}\left(u_{j}^{C}, u_{j}^{D}\right)+\sum_{r \in \mathscr{R}_{D}}\left|\gamma_{r}^{D}\right| \lambda_{r}\left(u_{j}^{D}\right),
$$

so that

$$
|g|(u)=\sum_{r \in \mathscr{R}_{D C} \backslash S_{1}}\left|\gamma_{r}^{D}\right| \lambda_{r}\left(u_{C}, u_{D}\right)+\sum_{r \in \mathscr{R}_{D}}\left|\gamma_{r}^{D}\right| \lambda_{r}\left(u_{D}\right) .
$$

Below, we introduce different quantities and functions such as $|g|$, related to debit type functions, and explain the intuition behind them. We call $|g|$ the "amplitude function" associated with the debit type function $g$. Then as usual, we may view $|g|$ as a real valued function defined on $\mathbb{R}^{2}$. Now, we make the following

\section{Assumption 2.5}

(C1) $F$ is locally Lipschitz and $F(y) \geq 0$ for $y=\left(y_{1}, y_{2}\right) \in \mathbb{R}^{2}$ such that $y_{1}=0$.

(C2) There exists $0<\rho_{C}<\infty$ such that $|y|>\rho_{C}$ yields $F(y)<0$ for all $y \in \mathbb{R}^{m}$.

(D1) $\theta(y)=0$ for $y \leq 0$.

(D2) $g$ is locally Lipschitz, and $|g|$ has at most linear growth w.r.t. its second variable: for all $\bar{c}>0$ there exists $M_{1}(\bar{c}) \geq 0$ such that, for $\left|y_{1}\right| \leq \bar{c},|g|\left(y_{1}, y_{2}\right) \leq M_{1}(\bar{c})\left(\left|y_{2}\right|+1\right)$.

Concerning the reactant $C$, Assumption 2.5 (C1) and (C2) correspond to Assumption 2.1. These conditions are met in particular, as soon as the rate of each fast reaction satisfies (C1) and (C2) in the place of $F$. Yet, there is difference with $D$. First of all, in order to ensure positivity for its concentration, we have introduced the function $\theta$ in Assumption 2.4. Moreover, the debit associated with $D$ involves a convolution product at the limit. It seems difficult to write an assumption similar to (C2) and we assume linear growth which is less general.

The upcoming result states that $\mathscr{A}^{\infty}$ is the generator of a unique process. It is a straightforward adaptation of the result of Kotelenez in [24], about well-posedness for the one-scale deterministic spatial model.

\section{Proposition 2.3}

Assume that:

(i) the rates of onsite reactions are polynomial and such that Assumption 2.5 holds,

(ii) $v_{0}=\left(v_{0}^{C}, v_{0}^{D}\right) \in C^{3}(I) \times C^{3}(I)$, 
(iii) $\rho_{C}, \rho_{D} \gg 1$ such that $\left\|v_{0}^{C}\right\|_{\infty}<\rho_{C}<\infty$ and $\left\|v_{0}^{D}\right\|_{\infty}<\rho_{D}<\infty$.

Then, (22) has a unique global mild solution $v:=v\left(t, v_{0}\right)$ satisfying

$$
v=\left(v_{C}, v_{D}\right) \in C\left(\mathbb{R}_{+} ; C^{3}(I) \times C^{3}(I)\right)
$$

and

$$
v(t)=\tilde{T}(t) v_{0}+\int_{0}^{t} \tilde{T}(t-s) R(v(s)) d s, t \geq 0
$$

Moreover,

$$
\begin{cases}v_{C}(t) \geq 0, \quad v_{D}(t) \geq 0, & t \geq 0 \\ \left\|v_{C}(t)\right\|_{\infty} \leq \rho_{C}, & t \geq 0 \\ \left\|v_{D}(t)\right\|_{\infty} \leq\left(\rho_{D}+1\right) \mathrm{e}^{a(0) M_{1}\left(\rho_{C}\right) t}, & t \geq 0\end{cases}
$$

A discretization of the limit. We define a discrete version $v^{N}=\left(v_{C}^{N}, v_{D}^{N}\right)$ of the limiting problem. Henceforth, $v=\left(v_{C}, v_{D}\right)$ denotes the solution to (22) given by Proposition 2.3. For $x \in I, t \geq 0$, we consider the system:

$$
\left\{\begin{array}{l}
\frac{\partial}{\partial t} v_{C}^{N}(t, x)=\Delta_{N} v_{C}^{N}(t, x)+F\left(v_{C}^{N}(t, x), v_{D}^{N}(t, x)\right) \\
\frac{\partial}{\partial t} v_{D}^{N}(t, x)=G\left(v_{C}^{N}(t, x), v_{D}^{N}(t, x)\right) \\
v_{C}^{N}(t, 0)=v_{C}^{N}(t, 1) \text { and } v_{D}^{N}(t, 0)=v_{D}^{N}(t, 1) \\
v_{C}^{N}(0, x)=P_{N} v_{C}(0) \text { and } v_{D}^{N}(0):=P_{N} v_{D}(0) .
\end{array}\right.
$$

Note that $v^{N}(0)=\widetilde{P}_{N} v(0)$, where we recall $\tilde{P}_{N} v:=\left(P_{N} v_{C}, P_{N} v_{D}\right)$. Using the operators introduced in Proposition 2.2, a compact version for this system of ODEs reads

$$
\left\{\begin{array}{l}
\frac{d}{d t} v^{N}(t)=\tilde{\Delta}_{N} v^{N}(t)+R\left(v^{N}(t), v^{N}(t)\right) \\
v^{N}(t, 0)=v^{N}(t, 1) \\
v^{N}(0)=\tilde{P}_{N} v(0) .
\end{array}\right.
$$

The next result gives a relation between the limiting problem and its discretization. Its proof is reported to Appendix A.2.

\section{Theorem 2.1}

If the rates of onsite reactions are polynomial and Assumption 2.5 holds, then (26) has a unique global mild solution $v^{N}=\left(v_{C}^{N}, v_{D}^{N}\right) \in C\left(\mathbb{R}_{+} ; \mathbb{Q}^{N} \times \mathbb{\square}^{N}\right)$ satisfying

$$
v^{N}(t)=\tilde{T}_{N}(t) \tilde{P}_{N} v(0)+\int_{0}^{t} \tilde{T}_{N}(t-s)\left(\begin{array}{l}
F\left(v^{N}(s)\right) \\
G\left(v^{N}(s)\right)
\end{array}\right) d s
$$

for all $t \geq 0$. Furthermore, for any fixed $T>0$,

$$
\begin{cases}\left\|v_{C}^{N}(t)\right\|_{\infty} \leq(\rho+1) / 2, & t \geq 0, \\ \left\|v_{D}^{N}(t)\right\|_{\infty} \leq \bar{c}_{D}=\bar{c}_{D}\left(M_{1}, T, a(0)\right), & 0 \leq t \leq T,\end{cases}
$$

and

$$
\sup _{t \in[0, T]}\left\|v^{N}(t)-v(t)\right\|_{\infty, \infty} \longrightarrow 0
$$

\subsubsection{Main result: the law of large numbers}

Now, we state and prove our main result.

\section{Theorem 2.2}

Consider a sequence $u^{N}=\left(u_{C}^{N}, u_{D}^{N}\right)$ of Markov processes starting at $u^{N}(0)=\left(u_{C}^{N}(0), u_{D}^{N}(0)\right)$, with infinitesimal generators $\mathscr{A}^{N}$ given by (7). Assume that the assumptions of Proposition 2.3 hold and:

(i) $N, \mu \longrightarrow \infty$ in such a way that $\mu^{-1} \log N \longrightarrow 0$,

(ii) $u^{N}(0) \geq 0$ and $\left\|u^{N}(0)-v(0)\right\|_{\infty, \infty} \longrightarrow 0$ in probability.

Then, for all $T>0$,

$$
\sup _{t \in[0, T]}\left\|u^{N}(t)-v(t)\right\|_{\infty, \infty} \rightarrow 0 \text { in probability. }
$$




\section{Proof of the main result}

Let $T>0$ be fixed. Our goal is to show that for all $\epsilon>0 \mathbb{P}\left\{\sup _{[0, T]}\left\|u^{N}(t)-v(t)\right\|_{\infty, \infty}>\epsilon\right\} \rightarrow 0$.

It follows from Theorem 2.1 that it is sufficient to prove that for all $\epsilon>0$

$$
\mathbb{P}\left\{\sup _{[0, T]}\left\|u^{N}(t)-v^{N}(t)\right\|_{\infty, \infty}>\epsilon\right\} \rightarrow 0 .
$$

The rest of the proof is divided in two principal steps. In the first step, we successively consider some martingales associated to our model (M6) and, an adequate truncation of our process in time, using the properties of these martingales. The aim is to work only with the truncated model in the place of the initial one. Next, we conclude using a Gronwall-Bellman argument.

\subsection{Accompanying martingales}

Different types of martingales are associated to jump Markov processes such as $u^{N}$. Before specifying some of them, we introduce:

Some useful notation. Let $j=1, \cdots, N$ and $u=\left(u_{C}, u_{D}\right) \in C_{p}(I) \times C_{p}(I)$ be fixed.

$-\lambda_{j}^{C}(u)\left(\right.$ resp. $\left.\lambda_{j}^{D}(u)\right)$ is the rate for fast (resp. slow) reactions on site $j$ :

$$
\begin{aligned}
& \lambda_{j}^{C}\left(u_{C}, u_{D}\right)=\mu\left(\sum_{r \in \Re_{C}} \lambda_{r}\left(u_{j}^{C}\right)+\sum_{r \in S_{1}} \lambda_{r}\left(u_{j}^{C}, u_{j}^{D}\right)+2 N^{2} u_{j}^{C}\right), \text { and } \\
& \lambda_{j}^{D}\left(u_{C}, u_{D}\right)=\sum_{r \in \Re_{D C} \backslash S_{1}} \lambda_{r}\left(u_{j}^{C}, u_{j}^{D}\right)+\sum_{r \in \Re_{D}} \lambda_{r}\left(u_{j}^{D}\right) .
\end{aligned}
$$

$-\lambda_{j}(u):=\lambda_{j}^{C}(u)+\lambda_{j}^{D}(u)$ is the total rate for reactions on site $j$.

$-\lambda^{N}(u):=\sum_{j=1}^{N} \lambda_{j}(u)$ is the total rate for reactions in the system:

$$
\begin{aligned}
\lambda^{N}\left(u_{C}, u_{D}\right)= & \mu \sum_{j=1}^{N}\left(\sum_{r \in \Re_{C}} \lambda_{r}\left(u_{j}^{C}\right)+\sum_{r \in S_{1}} \lambda_{r}\left(u_{j}^{C}, u_{j}^{D}\right)+2 N^{2} u_{j}^{C}\right) \\
& +\sum_{j=1}^{N}\left(\sum_{r \in \Re_{D C} \backslash S_{1}} \lambda_{r}\left(u_{j}^{C}, u_{j}^{D}\right)+\sum_{r \in \Re_{D}} \lambda_{r}\left(u_{j}^{D}\right)\right) .
\end{aligned}
$$

- When reaction rates are bounded, we set $\bar{\lambda}:=\max \left(\bar{\lambda}_{C}, \bar{\lambda}_{D}\right)$ where,

$$
\bar{\lambda}_{C}=\sum_{r \in \Re_{C} \cup S_{1}}\left\|\lambda_{r}\right\|_{\infty}, \quad \bar{\lambda}_{D}=\sum_{r \in\left(\mathfrak{R}_{D C} \backslash S_{1}\right) \cup \Re_{D}}\left\|\lambda_{r}\right\|_{\infty}
$$

$-\bar{\gamma}:=\max \left(\bar{\gamma}_{C}, \bar{\gamma}_{D}\right)$ with $\bar{\gamma}_{D}:=\max \left(\sum_{r \in \Re_{D C} \backslash S_{1}}\left|\gamma_{r}^{D}\right|, \sum_{r \in \Re_{D}}\left|\gamma_{r}^{D}\right|\right)$.

The notation $\bar{\gamma}_{C}$ is the counterpart for fast reactions.

Recall that the debit of a process is defined as the sum of its jumps weighted by the corresponding rates, over all its possible jump directions. Let

$$
\delta u_{j}^{N}(t):=u_{j}^{N}(t)-u_{j}^{N}\left(t^{-}\right)
$$

be the jump of $u_{j}^{N}$ at time $t$, and denote by $\left|\delta u_{j}^{N}(t)\right|$ the amplitude of that jump, where $\left|\left(y_{1}, y_{2}\right)\right|=\left|y_{1}\right|+\left|y_{2}\right|$. Firstly, we define the "amplitude" $\left|\Psi_{C}^{N}\right|_{j}$ (resp. $\left|\Psi_{D}^{N}\right|_{j}$ ) of the debit $\Psi_{j}^{N, C}$ (resp. $\Psi_{j}^{N, D}$ ), as the debit function of the process

$$
\left(\sum_{s \leq t}\left|\delta u_{j}^{N, C}(t)\right|\right)_{t \geq 0}\left[\operatorname{resp} \cdot\left(\sum_{s \leq t}\left|\delta u_{j}^{N, D}(t)\right|\right)_{t \geq 0}\right] .
$$


This notion of "amplitude" is perfectly adapted to any of the specific debit functions $\Delta_{N}, F, F_{1}^{N}$, or $G^{N}$. Especially, we have for the part related to $C$ :

$-\left|\Delta_{N}\right|_{j}\left(u_{C}\right)=N^{2}\left(u_{j-1}^{C}+2 u_{j}^{C}+u_{j+1}^{C}\right)$,

$-|F|_{j}\left(u_{C}, u_{D}\right)=\sum_{r \in \Re_{C}}\left|\gamma_{r}^{C}\right| \lambda_{r}\left(u_{j}^{C}\right)+\sum_{r \in S_{1}}\left|\gamma_{r}^{C}\right| \lambda_{r}\left(u_{j}^{C}, u_{j}^{D}\right)$,

$-\left|F_{1}^{N}\right|_{j}\left(u_{C}, u_{D}\right)=\frac{1}{\mu} \sum_{i=1}^{N} \sum_{r \in \Re_{D C} \backslash S_{1}}\left|\gamma_{i j}^{N}\right| \theta_{i j}^{r}\left(u_{i}^{C}, u_{i}^{D}\right) \lambda_{r}\left(u_{j}^{C}, u_{j}^{D}\right)$,

- $\left|\Psi_{C}^{N}\right|_{j}\left(u_{C}, u_{D}\right)=\left|\Delta_{N}\right|_{j}(u)+|F|_{j}(u)+\left|F_{1}^{N}\right|_{j}\left(u_{C}, u_{D}\right)$.

For the part related to the reactant $D$, we have

$-|g|_{i j}\left(u_{C}, u_{D}\right)=\sum_{r \in \Re_{D C} \backslash S_{1}}\left|\gamma_{r}^{D}\right| \theta_{i j}^{r}\left(u_{i}^{C}, u_{i}^{D}\right) \lambda_{r}\left(u_{j}^{C}, u_{j}^{D}\right)+\sum_{r \in \Re_{D}}\left|\gamma_{r}^{D}\right| \theta_{i j}^{r}\left(u_{i}^{C}, u_{i}^{D}\right) \lambda_{r}\left(u_{j}^{D}\right)$, for all $1 \leq i \leq N$.

- $\left|\Psi_{D}^{N}\right|_{j}\left(u_{C}, u_{D}\right)=\sum_{i=1}^{N}\left|\gamma_{i j}^{N}\right||g|_{i j}\left(u_{C}, u_{D}\right)=:\left|G^{N}\right|_{j}\left(u_{C}, u_{D}\right)$.

Then we set $\left|\Psi^{N}\right|_{j}(u):=\left|\Psi_{C}^{N}\right|_{j}(u)+\left|\Psi_{D}^{N}\right|_{j}(u)$ and $\left|\Psi^{N}\right|(u):=\sum_{j=1}^{N}\left|\Psi^{N}\right|_{j}(u)$.

We analogously define the "square amplitude" $\left|\Psi_{C}^{N}\right|_{j}^{2}\left(\operatorname{resp} .\left|\Psi_{D}^{N}\right|_{j}^{2}\right)$ of $\Psi_{j}^{N, C}\left(\operatorname{resp} . \Psi_{j}^{N, D}\right)$, as the debit function of the process

$$
\left(\sum_{s \leq t}\left|\delta u_{j}^{N, C}(t)\right|^{2}\right)_{t \geq 0}\left[\operatorname{resp} \cdot\left(\sum_{s \leq t}\left|\delta u_{j}^{N, D}(t)\right|^{2},\right)_{t \geq 0}\right] .
$$

Then,

$-\left|\Delta_{N}\right|_{j}^{2}\left(u_{C}\right):=N^{2}\left(u_{j-1}^{C}+2 u_{j}^{C}+u_{j+1}^{C}\right)$,

$-|F|_{j}^{2}\left(u_{C}, u_{D}\right)=\sum_{r \in \Re_{C}}\left|\gamma_{r}^{C}\right|^{2} \lambda_{r}\left(u_{j}^{C}\right)+\sum_{r \in S_{1}}\left|\gamma_{r}^{C}\right|^{2} \lambda_{r}\left(u_{j}^{C}, u_{j}^{D}\right)$,

$-\left|F_{1}^{N}\right|_{j}^{2}\left(u_{C}, u_{D}\right)=\frac{1}{\mu^{2}} \sum_{i=1}^{N} \sum_{r \in \Re_{D C} \backslash S_{1}}\left|\gamma_{i j}^{N}\right|^{2}\left|\theta_{i j}^{r}\left(u_{i}^{C}, u_{i}^{D}\right)\right|^{2} \lambda_{r}\left(u_{j}^{C}, u_{j}^{D}\right)$,

$-\left|\Psi_{C}^{N}\right|_{j}^{2}(u)=\left|\Delta_{N}\right|_{j}^{2}(u)+|F|_{j}^{2}(u)+\left|F_{1}^{N}\right|_{j}^{2}\left(u_{C}, u_{D}\right)$,

$-|g|_{i j}^{2}\left(u_{C}, u_{D}\right)=\sum_{r \in \Re_{D C} \backslash S_{1}}\left|\gamma_{r}^{D}\right|^{2}\left|\theta_{i j}^{r}\left(u_{i}^{C}, u_{i}^{D}\right)\right|^{2} \lambda_{r}\left(u_{j}^{C}, u_{j}^{D}\right)+\sum_{r \in \Re_{D}}\left|\gamma_{r}^{D}\right|^{2}\left|\theta_{i j}^{r}\left(u_{i}^{C}, u_{i}^{D}\right)\right|^{2} \lambda_{r}\left(u_{j}^{D}\right)$, for all $1 \leq i \leq N$,

$-\left|\Psi_{D}^{N}\right|_{j}^{2}\left(u_{C}, u_{D}\right)=\sum_{i=1}^{N}\left|\gamma_{i j}^{N}\right|^{2}|g|_{i j}^{2}\left(u_{C}, u_{D}\right)=:\left|G^{N}\right|_{j}^{2}\left(u_{C}, u_{D}\right)$

And we set $\left|\Psi^{N}\right|_{j}^{2}(u):=\left|\Psi_{C}^{N}\right|_{j}^{2}(u)+\left|\Psi_{D}^{N}\right|_{j}^{2}(u)$ and $\left|\Psi^{N}\right|^{2}(u):=\sum_{j=1}^{N}\left|\Psi^{N}\right|_{j}^{2}(u)$.

In addition, we introduce the very useful "square amplitude function" associated with the debit $\Psi^{N}$. It is defined by

$$
\left|\Psi^{N}\right|^{2}(u)=\sum_{j=1}^{N}\left|\Psi^{N}\right|_{j}^{2}(u) \mathbb{1}_{j} .
$$

We also denote it by $\left|\Psi^{N}\right|^{2}$, as the "square amplitude". Both functions are defined on $\mathbb{\boxplus}^{N} \times \mathbb{U}^{N}$. But still, if the former is $\mathbb{t}^{N}-$ valued, the latter has non-negative real values. In order to avoid any confusion in the rest of the article, the notation $\left|\Psi^{N}\right|^{2}$ refers to the "square amplitude function", unless another precision is made. 
Moreover, as for the "square amplitude" introduced first, the "square amplitude function" can be easily derived for each of the specific debit functions we have introduced above. For instance, the "square amplitude function" corresponding to $F$ is just

$$
|F|^{2}(u):=\sum_{j=1}^{N}|F|_{j}^{2}(u) \mathbb{1}_{j} .
$$

The so-called accompanying martingales. It is well known from [30] or Proposition 2.1 of [31] that, if the total reaction rate $\lambda^{N}$ and the global "amplitude" - that of the global debit $\Psi^{N}-$ of $u^{N}$ are bounded, or in other words if

$$
\sup _{u \in \mathbb{H}^{N} \times \mathbb{H}^{N}} \lambda^{N}(u)<\infty \quad \text { and } \sup _{u \in \mathbb{H}^{N} \times \mathbb{H}^{N}}\left|\Psi^{N}(u)\right|<\infty,
$$

then the processes defined by

$$
\left\{\begin{array}{l}
Z^{N}(t)=u^{N}(t)-u^{N}(0)-\int_{0}^{t} \Psi^{N}\left(u^{N}(s)\right) d s \\
Z_{C}^{N}(t):=u_{C}^{N}(t)-u_{C}^{N}(0)-\int_{0}^{t} \Psi_{C}^{N}\left(u^{N}(s)\right) d s \\
Z_{D}^{N}(t):=u_{D}^{N}(t)-u_{D}^{N}(0)-\int_{0}^{t} \Psi_{D}^{N}\left(u^{N}(s)\right)
\end{array}\right.
$$

are $\mathscr{F}_{t}^{N}=\mathscr{F}_{t}^{N, \mu}$-martingales, with $Z^{N}(t)=\left(Z_{C}^{N}(t), Z_{D}^{N}(t)\right)$ taking values in $\mathbb{T}^{N} \times \mathbb{H}^{N}$, for all $t \geq 0$. Note that (32) holds when reaction rates are bounded for instance. Furthermore, if $\tau$ is a $\mathscr{F}_{t}^{N}$-stopping time such that

$$
\sup _{[0, T]}\left\|u^{N}(t \wedge \tau)\right\|_{\infty, \infty} \leq C(T, N, \mu)<\infty,
$$

then $u^{N}(t \wedge \tau)$ has a bounded total jump rate, and $Z^{N}(t \wedge \tau), Z_{C}^{N}(t \wedge \tau)$ and $Z_{D}^{N}(t \wedge \tau)$ are martingales. In all these, $t \wedge \tau$ denotes the infimum between $t$ and $\tau$.

The following result is similar to Lemma 2.2, p.8. in [6]. It presents a first type of martingales related to $u^{N}$. We give its proof in Appendix A.3.

\section{Lemma 3.1}

Assume there exists $\tau$ satisfying (34). Then, for all $j \in\{1, \cdots, N\}$ and all $t \geq 0$, the following define mean zero $\mathscr{F}_{t}^{N}$-martingales:

$$
\begin{aligned}
& \text { (Mg1) } \sum_{s \leq t \wedge \tau}\left[\delta u_{j}^{N, C}(s)\right]^{2}-\frac{1}{\mu} \int_{0}^{t \wedge \tau}\left(|\Delta|_{j}^{2}+|F|_{j}^{2}+\mu\left|F_{1}^{N}\right|^{2}\right)\left(u^{N}(s)\right) d s, \\
& \text { (Mg2)-(Mg3) } \sum_{s \leq t \wedge \tau}\left[\delta u_{j}^{N, C}(s)\right]\left[\delta u_{j \pm 1}^{N, C}(s)\right]+\frac{1}{\mu} \int_{0}^{t \wedge \tau} N^{2}\left(u_{j}^{N, C}(s)+u_{j \pm 1}^{N, C}(s)\right) d s, \\
& \text { (Mg4) } \sum_{s \leq t \wedge \tau}\left[\delta u_{j}^{N, D}(s)\right]^{2}-\int_{0}^{t \wedge \tau}\left|\Psi_{j}^{N, D}\right|^{2}\left(u^{N}(s)\right) d s .
\end{aligned}
$$

We move on to the second martingale type. The result is a variant of Lemma 2.16, p.19 in [6], and Lemma 1.1 in [25]. Find the proof in Appendix A.3.

\section{Lemma 3.2}

Consider $\varphi \in \mathbb{M}^{N}$ and $\tau$ satisfying (34). Then for $t \geq 0$, the following are mean 0 martigales:

$$
\begin{aligned}
& \text { (Mg5) } \sum_{s \leq t \wedge \tau}\left[\delta\left\langle Z_{C}^{N}(s), \varphi\right\rangle_{2}\right]^{2}-\frac{1}{N \mu} \int_{0}^{t \wedge \tau}\left[\left\langle u_{C}^{N}(s),\left(\nabla_{N}^{+} \varphi\right)^{2}+\left(\nabla_{N}^{-} \varphi\right)^{2}\right\rangle_{2}+\left\langle\left(|F|^{2}+\mu\left|F_{1}^{N}\right|^{2}\right)\left(u^{N}(s)\right), \varphi^{2}\right\rangle_{2}\right] d s, \\
& \text { (Mg6) } \sum_{s \leq t \wedge \tau}\left[\delta\left\langle Z_{D}^{N}(s), \varphi(s)\right\rangle_{2}\right]^{2}-\frac{1}{N} \int_{0}^{t \wedge \tau}\left\langle\left|\Psi_{D}^{N}\right|^{2}\left(u^{N}(s)\right), \varphi^{2}\right\rangle_{2} d s .
\end{aligned}
$$




\subsection{Truncation}

We define a stopping time satisfying (34). Set

$$
\tau:=\tau\left(N, \epsilon_{0}\right)=\inf \left\{t \geq 0:\left\|u^{N}(t)-v^{N}(t)\right\|_{\infty, \infty}>\epsilon_{0}\right\},
$$

for fixed $\epsilon_{0} \in[0,1]$. By definition of $\tau$, the process $u^{N}$ exists and takes finite value on $[0, \tau]$. Now, define $\bar{u}^{N}=$ $\left(\bar{u}_{C}^{N}, \bar{u}_{D}^{N}\right)$ by

$$
\begin{cases}\bar{u}^{N}(t)=u^{N}(t \wedge \tau) & \text { for } 0 \leq t \leq \tau \leq \infty \\ \bar{u}^{N}(t)=u^{N}(t \wedge \tau)+\int_{t \wedge \tau}^{t}\left(\tilde{\Delta}_{N} \bar{u}^{N}(s)+R^{N}\left(\bar{u}^{N}(s)\right)\right) d s & \text { for } \tau<t<\infty\end{cases}
$$

where $R^{N}(u):=\left(\begin{array}{c}F(u) \\ G^{N}(u)\end{array}\right)$ for $u \in C_{p}(I) \times C_{p}(I)$. The process $\bar{u}^{N}$ is obtained by running $u^{N}$ until time $\tau$ and then running it deterministically afterwards, if $\tau<\infty$.

Note that $\tau$ is a stopping time such that

$\mathbb{P}\left\{\sup _{[0, T]}\left\|u^{N}(t)-v^{N}(t)\right\|_{\infty, \infty}>\epsilon_{0}\right\} \leq \mathbb{P}\left\{\sup _{[0, T]}\left\|u^{N}(t \wedge \tau)-v^{N}(t \wedge \tau)\right\|_{\infty, \infty} \geq \epsilon_{0}\right\} \leq \mathbb{P}\left\{\sup _{[0, T]}\left\|\bar{u}^{N}(t)-v^{N}(t)\right\|_{\infty, \infty} \geq \epsilon_{0}\right\}$.

Therefore, it follows from (31) that showing

$$
\mathbb{P}\left\{\sup _{[0, T]}\left\|\bar{u}^{N}(t)-v^{N}(t)\right\|_{\infty, \infty}>\epsilon_{0}\right\} \rightarrow 0
$$

is sufficient for proving the main result.

Boundedness. From Proposition 2.3, we know that $\|v(t)\|_{\infty, \infty}<\rho_{T}$ with $\rho_{T}=\rho_{C}+\left(\rho_{D}+1\right) \mathrm{e}^{a(0) M_{1}\left(\rho_{C}\right) T}$ for all $t \in[0, T]$. Since we are assuming $\left\|u^{N}(0)-v(0)\right\|_{\infty, \infty} \rightarrow 0$ in probability, we may, by conditioning on $\left\|u^{N}(0)\right\|_{\infty, \infty}<$ $\rho_{T}+1$ if necessary, assume without loss of generality that

$$
\left\|u^{N}(0)\right\|_{\infty, \infty}<\rho_{T}+1 \text { for all } N
$$

From Theorem 2.1, we also have

$$
\sup _{t \in[0, T]}\left\|v_{C}^{N}(t)\right\|_{\infty} \leq \rho_{C}, \sup _{t \in[0, T]}\left\|v_{D}^{N}(t)\right\|_{\infty} \leq\left(\rho_{D}+1\right) \mathrm{e}^{a(0) M_{1}\left(\rho_{C}\right) T} .
$$

Therefore, by definition of $\tau$, for $\epsilon_{0} \leq 1$,

$$
\left\|u_{D}^{N}(t \wedge \tau)\right\|_{\infty, \infty}<\rho_{C}+1, \quad\left\|u_{D}^{N}(t \wedge \tau)\right\|_{\infty, \infty}<\left(\rho_{D}+1\right) \mathrm{e}^{a(0) M_{1}\left(\rho_{C}\right) T}+1 \quad \text { for } 0 \leq t \leq T .
$$

Thanks to Assumption 2.5, we obtain

$$
\left\|\bar{u}^{N}(t)\right\|_{\infty, \infty} \leq \tilde{\rho}_{T}=\rho_{C}+1+\left(\rho_{D}+2\right) \mathrm{e}^{a(0) M_{1}\left(\rho_{C}+1\right) T} \quad \text { for } 0 \leq t \leq T .
$$

Indeed, given (38), we prove (39) if for $\tau<t \leq T$, we show the inequalities

$$
\begin{cases}\left\|\bar{u}_{C}^{N}(t)\right\|_{\infty} \leq \rho_{C}+1 & (C \star) \\ \left\|\bar{u}_{D}^{N}(t)\right\|_{\infty} \leq\left(\rho_{D}+2\right) \mathrm{e}^{2 a(0) M_{1}\left(\rho_{C}+1\right) T} & (D \star)\end{cases}
$$

Suppose that $\bar{u}_{j}^{N, C}(t)>\rho_{C}+1$ for some $t \in[\tau, T], 1 \leq j \leq N$. Since $\bar{u}_{C}^{N}(t) \in \mathbb{H}^{N}$, there is $1 \leq i \leq N$ and $t_{0}$ satisfying

$$
\bar{u}_{i}^{N, C}\left(t_{0}\right)=\sup _{s \in[\tau, T]}\left\|\bar{u}_{C}^{N}(s)\right\|_{\infty} \geq \bar{u}_{j}^{N, C}(t)>\rho_{C}+1 .
$$

Therefore, Assumption 2.5 (i) yields

$$
F\left(\bar{u}_{i}^{N}\left(t_{0}\right)\right)=F\left(\bar{u}_{i}^{N, C}\left(t_{0}\right), \bar{u}_{i}^{N, D}\left(t_{0}\right)\right)<0
$$


Moreover

$$
\Delta_{N} \bar{u}_{i}^{N, C}\left(t_{0}\right)=\bar{u}_{i+1}^{N, C}\left(t_{0}\right)-2 \bar{u}_{i}^{N, C}\left(t_{0}\right)+\bar{u}_{i-1}^{N, C}\left(t_{0}\right)<0 .
$$

Combining these two arguments with (35), we get

$$
\frac{d}{d t} \bar{u}_{i}^{N, C}\left(t_{0}\right)=\Delta_{N} \bar{u}_{i}^{N, C}\left(t_{0}\right)+F\left(\bar{u}_{i}^{N}\left(t_{0}\right)\right)<0 .
$$

By (38) $t_{0}>\tau$ and necessarily $\frac{d}{d t} \bar{u}_{i}^{N, C}\left(t_{0}\right) \geq 0$ in contradiction with the above. Thus, $(C \star)$ follows.

Now, let $\tau<t \leq T$ and $1 \leq i \leq N$ be fixed. Let $\tau \leq s \leq t$. Observing that $\mid g_{i j}\left(u^{N}(s)\left|\leq\left\||g|\left(u^{N}(s)\right)\right\|_{\infty}\right.\right.$, Assumption 2.5 (ii) and $(C \star)$ yield

$$
\begin{aligned}
\left|G^{N}\left(\bar{u}_{i}^{N, D}(s)\right)\right| & \leq \sum_{j=1}^{N}\left|\gamma _ { i j } ^ { N } \left\|g _ { i j } ( u ^ { N } ( s ) ) \left|\leq \sum_{j=1}^{N} \frac{a(0)}{N}\left\||g|\left(u^{N}(s)\right)\right\|_{\infty}\right.\right.\right. \\
& \leq a(0) M_{1}\left(\rho_{C}+1\right)\left(\left\|\bar{u}_{D}^{N}(s)\right\|_{\infty}+1\right) .
\end{aligned}
$$

Thus, $\left\|G^{N}\left(\bar{u}_{D}^{N}(s)\right)\right\|_{\infty} \leq a(0) M_{1}\left(\rho_{C}+1\right)\left(\left\|\bar{u}_{D}^{N}(s)\right\|_{\infty}+1\right)$, and, (35) with (38) lead to

$$
\begin{aligned}
\left\|\bar{u}_{D}^{N}(t)\right\|_{\infty} & \leq\left\|u_{D}^{N}(t \wedge \tau)\right\|_{\infty}+\int_{\tau}^{t}\left\|G^{N}\left(\bar{u}_{i}^{N, D}(s)\right)\right\|_{\infty} d s \\
& \leq\left(\rho_{D}+1\right) \mathrm{e}^{a(0) M_{1}\left(\rho_{C}\right) T}+1+M_{1}\left(\rho_{C}+1\right) a(0) \int_{\tau}^{t}\left(\left\|\bar{u}_{D}^{N}(s)\right\|_{\infty}+1\right) d s
\end{aligned}
$$

The result follows from Gronwall lemma. $(D \star)$ follows, and (39) is proved.

In the rest of the article, we consider $\bar{c}$ as a generic constant depending on $\tilde{\rho}_{T}$ and $T$.

\section{Remark 3.1}

As we are considering the truncated process $\bar{u}^{N}$ in the following, we consider that reaction rates are bounded and Lipschitz as functions defined on $\mathbb{R}^{2}$. Indeed, thanks to (39), we know that the family $\left\{\bar{u}^{N}(t), t \geq 0\right\}_{N}$ lies in the bounded set

$$
\mathfrak{S}_{\tilde{\rho}_{T}}:=\left\{u \in \mathbb{M}^{N} \times \mathbb{M}^{N}:\|u\|_{\infty, \infty} \leq \tilde{\rho}_{T}\right\} .
$$

Thus $\left\{\bar{u}^{N}(t, x), t \geq 0, x \in I\right\}_{N} \subset \bar{B}\left(0, \tilde{\rho}_{T}\right)$, where $\bar{B}\left(0, \tilde{\rho}_{T}\right)$, the closed ball of $\mathbb{R}^{2}$ of radius $\tilde{\rho}_{T}$, and centered at zero. Now, since reaction rates are functions of the concentrations of the truncated process, we only consider their restrictions to the compact $\bar{B}\left(0, \tilde{\rho}_{T}\right)$. These latter are bounded and Lipschitz, as the rates are polynomial. Their bounds depend on $\tilde{\rho}_{T}$ and are ت also denoted $\bar{\lambda}\left(\tilde{\rho}_{T}\right)$.

We have the following result, proved in Appendix A.4.

\section{Proposition 3.1}

(i) The debit functions $F, F_{1}^{N}, G$ and $G^{N}$ are Lipschitz.

(ii) For all $u \in C(I) \times C(I),\left\|G^{N}(u)-G(u)\right\|_{\infty} \longrightarrow 0$.

Martingale and jumps. The stopping time $\tau$ satisfies (34). Thus,

$$
Z^{N}(t \wedge \tau):=u^{N}(t \wedge \tau)-u^{N}(0)-\int_{0}^{t \wedge \tau}\left(\tilde{\Delta}_{N} u^{N}(s)+R^{N}\left(u^{N}(s)\right)\right) d s
$$

is a mean 0 martingale. From the definition of $\bar{u}^{N}$,

$$
\bar{u}^{N}(t)=u^{N}(0)+\int_{0}^{t}\left(\tilde{\Delta}_{N} \bar{u}^{N}(s)+R^{N}\left(\bar{u}^{N}(s)\right)\right) d s+Z^{N}(t \wedge \tau) .
$$

In fact, if $t \leq \tau$, then

$$
\begin{aligned}
\bar{u}^{N}(t) & -u^{N}(0)-\int_{0}^{t}\left(\tilde{\Delta}_{N} \bar{u}^{N}(s)+R^{N}\left(\bar{u}^{N}(s)\right)\right) d s \\
& :=u^{N}(t \wedge \tau)-u^{N}(0)-\int_{0}^{t}\left(\tilde{\Delta}_{N} u^{N}(s \wedge \tau)+R^{N}\left(u^{N}(s \wedge \tau)\right)\right) d s \\
& =u^{N}(t \wedge \tau)-u^{N}(0)-\int_{0}^{t \wedge \tau}\left(\tilde{\Delta}_{N} u^{N}(s)+R^{N}\left(u^{N}(s)\right)\right) d s=Z^{N}(t \wedge \tau) .
\end{aligned}
$$


Otherwise $t>\tau$ and, since $\bar{u}^{N}$ and $u^{N}$ are the same before time $\tau$,

$$
\begin{aligned}
\bar{u}^{N}(t) & -u^{N}(0)-\int_{0}^{t}\left(\tilde{\Delta}_{N} \bar{u}^{N}(s)+R^{N}\left(\bar{u}^{N}(s)\right)\right) d s \\
& :=u^{N}(t \wedge \tau)+\int_{t \wedge \tau}^{t}\left(\tilde{\Delta}_{N} \bar{u}^{N}(s)+R^{N}\left(\bar{u}^{N}(s)\right)\right) d s-u^{N}(0)-\int_{0}^{t}\left(\tilde{\Delta}_{N} \bar{u}^{N}(s)+R^{N}\left(\bar{u}^{N}(s)\right)\right) d s \\
& =u^{N}(t \wedge \tau)-u^{N}(0)-\int_{0}^{t \wedge \tau}\left(\tilde{\Delta}_{N} \bar{u}^{N}(s)+R^{N}\left(\bar{u}^{N}(s)\right)\right) d s \\
& =u^{N}(t \wedge \tau)-u^{N}(0)-\int_{0}^{t \wedge \tau}\left(\tilde{\Delta}_{N} u^{N}(s)+R^{N}\left(u^{N}(s)\right)\right) d s=Z^{N}(t \wedge \tau) .
\end{aligned}
$$

Also, setting $\delta f:=\left(\delta f_{j}\right)_{1 \leq j \leq N}$ for $f \in \mathbb{T}^{N}$, the jumps related to $u^{N}$ and $\bar{u}^{N}$ satisfy

$$
\left\{\begin{array}{l}
\left\|\delta \bar{u}_{C}^{N}(t)\right\|_{\infty}=\left\|\delta Z_{C}^{N}(t \wedge \tau)\right\|_{\infty}=\left\|\delta u_{C}^{N}(t \wedge \tau)\right\|_{\infty} \leq\left(1 \vee \bar{\gamma}_{C}\right) \mu^{-1} \leq \gamma \mu^{-1} \\
\left\|\delta \bar{u}_{D}^{N}(t)\right\|_{\infty}=\left\|\delta Z_{D}^{N}(t \wedge \tau)\right\|_{\infty}=\left\|\delta u_{D}^{N}(t \wedge \tau)\right\|_{\infty} \leq \bar{\gamma}_{D} a(0) N^{-1} \leq \gamma N^{-1}
\end{array}\right.
$$

for all $t \geq 0$, for some constant $\gamma>0$. We refer to (12) in particular for the bound related to $D$.

\subsection{A Gronwall-Bellman argument}

We want to study the difference

$$
\bar{u}^{N}(t)-v^{N}(t)=\left(\bar{u}_{C}^{N}(t)-v_{C}^{N}(t), \bar{u}_{D}^{N}(t)-v_{D}^{N}(t)\right) .
$$

Using variation of constant at (40), we get

$$
\bar{u}^{N}(t)=\tilde{T}_{N}(t) u^{N}(0)+\int_{0}^{t} \tilde{T}_{N}(t-s)\left(\begin{array}{c}
F\left(\bar{u}^{N}(s)\right) \\
G^{N}\left(\bar{u}^{N}(s)\right)
\end{array}\right) d s+Y^{N}(t),
$$

where $Y^{N}(t)=\int_{0}^{t} \tilde{T}_{N}(t-s) d Z^{N}(s \wedge \tau)$. Note that $Z^{N}\left(s \wedge \tau, \frac{j}{N}\right):=\left(\begin{array}{l}Z_{C}^{N}\left(s \wedge \tau, \frac{j}{N}\right) \\ Z_{D}^{N}\left(s \wedge \tau, \frac{j}{N}\right)\end{array}\right), 1 \leq j \leq N$, is of bounded variation in s, and $\widetilde{T}_{N}$ may be viewed as a $2 N \times 2 N$ matrix-valued function. $T_{N}$ is indeed identified to an $N \times N$ matrix-valued function. Hence, $Y^{N}\left(t, \frac{j}{N}\right), 1 \leq j \leq N$, is defined as a Stieltjes integral. From the mild forms of $v^{N}$ and $\bar{u}^{N}$ at (27) and (42) respectively,

$$
\bar{u}^{N}(t)-v^{N}(t)=\tilde{T}_{N}(t)\left(u^{N}(0)-v^{N}(0)\right)+\int_{0}^{t} \tilde{T}_{N}(t-s)\left(\begin{array}{c}
F\left(\bar{u}^{N}(s)\right)-F\left(v^{N}(s)\right) \\
G^{N}\left(\bar{u}^{N}(s)\right)-G\left(v^{N}(s)\right)
\end{array}\right)+Y^{N}(t) .
$$

Since the semigroup $\tilde{T}_{N}(t)$ is of contraction,

$$
\begin{gathered}
\left\|\bar{u}^{N}(t)-v^{N}(t)\right\|_{\infty, \infty} \leqslant\left\|u^{N}(0)-v^{N}(0)\right\|_{\infty, \infty}+\left\|Y^{N}(t)\right\|_{\infty, \infty} \\
+\int_{0}^{t}\left\|\left(\begin{array}{c}
F\left(\bar{u}^{N}(s)\right)-F\left(v^{N}(s)\right) \\
G^{N}\left(\bar{u}^{N}(s)\right)-G^{N}\left(v^{N}(s)\right)
\end{array}\right)\right\|_{\infty, \infty}+\left\|\left(\begin{array}{c}
0 \\
G^{N}\left(v^{N}(s)\right)-G\left(v^{N}(s)\right)
\end{array}\right)\right\|_{\infty, \infty} d s .
\end{gathered}
$$

We have

$$
\left\|\left(\begin{array}{c}
F\left(\bar{u}^{N}(s)\right)-F\left(v^{N}(s)\right) \\
G^{N}\left(\bar{u}^{N}(s)\right)-G^{N}\left(v^{N}(s)\right)
\end{array}\right)\right\|_{\infty, \infty} \leq 2 L\left\|\bar{u}^{N}(s)-v^{N}(s)\right\|_{\infty, \infty}
$$


and

$$
\begin{aligned}
& \left\|\left(G^{N}\left(v^{N}(s)\right)-G\left(v^{N}(s)\right)\right)\right\|_{\infty, \infty} \\
& =\left\|G^{N}\left(v^{N}(s)\right)-G\left(v^{N}(s)\right)\right\|_{\infty} \\
& \leq\left\|G^{N}\left(v^{N}(s)\right)-G^{N}(v(s))\right\|_{\infty}+\left\|G^{N}(v(s))-G(v(s))\right\|_{\infty} \\
& \quad+\left\|G(v(s))-G\left(v^{N}(s)\right)\right\|_{\infty} \\
& \quad \leq 2 L\left\|v^{N}(s)-v(s)\right\|_{\infty, \infty}+\left\|G^{N}(v(s))-G(v(s))\right\|_{\infty}
\end{aligned}
$$

where $L$ is a Lipschitz constant common to $F, G$ and $G^{N}$. Therefore,

$$
\begin{aligned}
&\left\|\bar{u}^{N}(t)-v^{N}(t)\right\|_{\infty, \infty} \\
& \leq\left\|u^{N}(0)-v^{N}(0)\right\|_{\infty, \infty}+\left\|Y^{N}(t)\right\|_{\infty, \infty}+2 L \int_{0}^{t}\left\|\bar{u}^{N}(s)-v^{N}(s)\right\|_{\infty, \infty} d s \\
& \quad+\int_{0}^{t}\left(2 L\left\|v^{N}(s)-v(s)\right\|_{\infty, \infty}+\left\|G^{N}(v(s))-G(v(s))\right\|_{\infty}\right) d s .
\end{aligned}
$$

Taking the supremum in time on $[0, T]$ and applying Gronwall lemma, we obtain

$$
\begin{aligned}
& \sup _{[0, T]}\left\|\bar{u}^{N}(t)-v^{N}(t)\right\|_{\infty, \infty} \\
& \leq\left\{\left\|u^{N}(0)-v^{N}(0)\right\|_{\infty, \infty}+\sup _{[0, T]}\left\|Y^{N}(t)\right\|_{\infty, \infty}\right. \\
&\left.+2 L T \sup _{[0, T]}\left\|v^{N}(t)-v(t)\right\|_{\infty, \infty}+\int_{0}^{T}\left\|G^{N}(v(s))-G(v(s))\right\|_{\infty} d s\right\} \times \mathrm{e}^{2 L T} .
\end{aligned}
$$

Now, by assumption,

$$
\left\|u^{N}(0)-v^{N}(0)\right\|_{\infty, \infty} \leq\left\|u^{N}(0)-v(0)\right\|_{\infty, \infty}+\left\|\tilde{P}_{N} v(0)-v(0)\right\|_{\infty, \infty} \rightarrow 0
$$

in probability. Also, $\int_{0}^{T}\left\|G^{N}(v(s))-G(v(s))\right\|_{\infty} \longrightarrow 0$, thanks to Proposition 3.1 and dominated convergence.

Moreover, Theorem 2.1 yields $\sup _{[0, T]}\left\|v^{N}(t)-v(t)\right\|_{\infty, \infty} \longrightarrow 0$.

Finally, we claim that:

$$
\sup _{[0, T]}\left\|Y^{N}(t)\right\|_{\infty, \infty} \longrightarrow 0 \text { in probability. }
$$

The proof of Theorem 2.2 is completed, if our claim is justified. We write

$$
Y^{N}(t)=\int_{0}^{t} \tilde{T}_{N}(t-s) d\left(\begin{array}{c}
Z_{C}^{N}(s \wedge \tau) \\
Z_{D}^{N}(s \wedge \tau)
\end{array}\right)=\left(\begin{array}{c}
\int_{0}^{t} T_{N}(t-s) d Z_{C}^{N}(s \wedge \tau) \\
Z_{D}^{N}(t \wedge \tau)
\end{array}\right)=:\left(\begin{array}{c}
Y_{C}^{N}(t) \\
Y_{D}^{N}(t)
\end{array}\right)
$$

where $Z_{C}^{N}(t \wedge \tau)$ and $Z_{D}^{N}(t \wedge \tau)$ are $\mathbb{\boxplus}^{N}$-valued martingales. It follows that

$$
\sup _{[0, T]}\left\|Y^{N}(t)\right\|_{\infty, \infty} \leq \sup _{[0, T]}\left\|Y_{C}^{N}(t)\right\|_{\infty}+\sup _{[0, T]}\left\|Z_{D}^{N}(t \wedge \tau)\right\|_{\infty} .
$$

Therefore, it is sufficient to show that each term on the r.h.s. of the inequality converges to zero in probability. For that purpose, we need the subsequent results, whose proofs are differed to Appendix A.5.

\section{Lemma 3.3}

(Lemma 4.3, [7]) Set $f=N 1_{j}$. Then $\left\langle\left(\nabla_{N}^{+} T_{N}(t) f\right)^{2}+\left(\nabla_{N}^{-} T_{N}(t) f\right)^{2}+\left(T_{N}(t) f\right)^{2}, 1\right\rangle_{2} \leq$ $h_{N}(t)$, with $\int_{0}^{t} h_{N}(s) d s \leq K N+t$. 


\section{Lemma 3.4}

(Lemma $4.4,[7])$ Let $m(t)$ be a bounded martingale of finite variation defined on $\left[t_{0}, t_{1}\right]$, with $m\left(t_{0}\right)=0$, and satisfying:

(i) $m$ is right-continuous with left limits.

(ii) $|\delta m(t)| \leq 1$ for $t_{0} \leq t \leq t_{1}$.

(iii) $\sum_{t_{0} \leq s \leq t}[\delta m(s)]^{2}-\int_{t_{0}}^{t} g(s) d s$ is a mean 0 martingale with $0 \leq g(s) \leq h(s)$, where $h(s)$ is a bounded deterministic function and $g(s)$ is $\mathscr{F}_{t}^{N}$-adapted.

Then,

$$
\mathbb{E}\left[\mathrm{e}^{m\left(t_{1}\right)}\right] \leq \exp \left(\frac{3}{2} \int_{t_{0}}^{t_{1}} h(s) d s\right) .
$$

Let us go back to the proof of 44 .

\subsubsection{Martingale continuous component term}

We want to show that $\mathbb{P}\left\{\sup _{[0, T]}\left\|Y_{C}^{N}(t)\right\|_{\infty}>\epsilon_{0}\right\} \rightarrow 0$.

Fix $\bar{t} \in(0, T]$ and $j \in\{1, \cdots, N\}$. For $0 \leq t \leq \bar{t}$, set

$$
f:=N 1_{j}, \text { and } \bar{m}_{C}(t):=\left\langle\int_{0}^{t} T_{N}(\bar{t}-s) d Z_{C}^{N}(s \wedge \tau), f\right\rangle_{2} .
$$

Then, $\bar{m}_{C}:=\left\{\bar{m}_{C}(t), 0 \leq t \leq \bar{t}\right\}$ is a mean zero martingale such that

$$
\begin{aligned}
\bar{m}_{C}(\bar{t}) & =N \int_{0}^{1} \int_{0}^{\bar{t}} T_{N}(\bar{t}-s) d Z_{C}^{N}(s \wedge \tau, x) 1_{j}(x) d x \\
& =\int_{0}^{\bar{t}} T_{N}(\bar{t}-s) d Z_{C}^{N}\left(s \wedge \tau, \frac{j}{N}\right)=Y_{C}^{N}\left(\bar{t}, \frac{j}{N}\right) .
\end{aligned}
$$

We need the additional upcoming result, that we prove in Appendix A.5.

\section{Lemma 3.5}

(A third type of accompanying martingale)

$$
\begin{aligned}
& \sum_{s \leq t \wedge \tau}\left[\delta \bar{m}_{C}(s)\right]^{2} \\
& -\frac{1}{N \mu} \int_{0}^{t \wedge \tau}\left[\left\langle u_{C}^{N}(s),\left(\nabla_{N}^{+} T_{N}(\bar{t}-s) f\right)^{2}+\left(\nabla_{N}^{-} T_{N}(\bar{t}-s) f\right)^{2}\right\rangle_{2}\right. \\
& \left.+\left\langle\left(|F|^{2}+\mu\left|F_{1}^{N}\right|^{2}\right)\left(u^{N}(s)\right),\left(T_{N}(\bar{t}-s) f\right)^{2}\right\rangle_{2}\right] d s
\end{aligned}
$$

defines a mean 0 càdlàg martingale for $0 \leq t \leq \bar{t}$.

\section{Remark 3.2}

In [7], Blount uses this result and claims that it is a consequence of Lemma 3.2, whose proof uses Lemma 3.1. We have not been able to reproduce his proof. Our proof uses Lemma 3.1 directly.

From (2.2), we know that $T_{N}(t)$ is a contraction on $\left(\mathbb{M}^{N},\|\cdot\|_{\infty}\right)$. Thus, (41) yields

$$
\begin{aligned}
\left|\delta \bar{m}_{C}(t)\right| & =\left|T_{N}(\bar{t}-t) \delta Z_{C}^{N}\left(t \wedge \tau, \frac{j}{N}\right)\right| \leq\left\|T_{N}(\bar{t}-t) \delta Z_{C}^{N}(t \wedge \tau)\right\|_{\infty} \\
& \leq\left\|\delta Z_{C}^{N}(t \wedge \tau)\right\|_{\infty}=\left\|\delta u_{C}^{N}(t \wedge \tau)\right\|_{\infty} \leq \gamma \mu^{-1} .
\end{aligned}
$$

For $\bar{\theta} \in\left[0, \gamma^{-1}\right]$, the process $m_{C}$ defined by

$$
m_{C}(t):=\bar{\theta} \mu \bar{m}_{C}(t) \quad \text { for } 0 \leq t \leq \bar{t}
$$


is a mean 0 càdlàg martingale such that $\left|\delta m_{C}(t)\right|=\bar{\theta} \mu\left|\delta \bar{m}_{C}(s)\right| \leq 1$. Thus,

$$
\sum_{s \leq t \wedge \tau}\left[\delta m_{C}(s)\right]^{2}-\int_{0}^{t \wedge \tau} g_{C}^{N}(s) d s
$$

is a mean 0 càdlàg martingale thanks to Lemma 3.5, where for $0 \leq s \leq t \wedge \tau$,

$$
\begin{aligned}
g_{C}^{N}(s)= & \frac{\bar{\theta}^{2} \mu}{N}\left[\left\langle u_{C}^{N}(s),\left(\nabla_{N}^{+} T_{N}(\bar{t}-s) f\right)^{2}+\left(\nabla_{N}^{-} T_{N}(\bar{t}-s) f\right)^{2}\right\rangle_{2}\right. \\
& \left.+\left\langle\left(|F|^{2}+\mu\left|F_{1}^{N}\right|^{2}\right)\left(u^{N}(s)\right),\left(T_{N}(\bar{t}-s) f\right)^{2}\right\rangle_{2}\right]
\end{aligned} .
$$

Since $u_{C}^{N}$ is positive and reaction rates are bounded, (38) and Lemma 3.3 yield

$$
\begin{aligned}
g_{C}^{N}(s) & \leq \frac{\bar{\theta}^{2} \mu}{N} \bar{c}\left\langle 1,\left(\nabla_{N}^{+} T_{N}(\bar{t}-s) f\right)^{2}+\left(\nabla_{N}^{-} T_{N}(\bar{t}-s) f\right)^{2}+\left(T_{N}(\bar{t}-s) f\right)^{2}\right\rangle_{2} \\
& \leq \frac{\bar{\theta}^{2} \mu}{N} \bar{c} h_{1}^{N, C}(\bar{t}-s)
\end{aligned}
$$

where we know from (55) that we can take $h_{1}^{N, C}(t)=1+4 \sum_{m>0} \mathrm{e}^{-2 \beta_{m, N}(\bar{t}-t)}\left(\beta_{m, N}+1\right)$, and it satisfies $\int_{0}^{t} h_{1}^{N, C}(\bar{t}-$ $s) d s \leq K N+t$, for $0 \leq t \leq \bar{t}$. Hence, $g_{C}^{N}$ is an $\mathscr{F}_{t}^{N}$-adapted process such that

$$
0 \leq g_{C}^{N}(s) \leq h_{C}^{N}(s),
$$

where $h_{C}^{N}(s)=\bar{c} \frac{\bar{\theta}^{2} \mu}{N} h_{1}^{N, C}(\bar{t}-s)$ is a bounded deterministic function on $[0, \bar{t}]$. Since $N \rightarrow \infty$ and $\bar{t} \leq T<\infty$, we may assume $\bar{t} N^{-1} \leq 1$ and get

$$
\int_{0}^{\bar{t}} h_{C}^{N}(s) d s \leq \bar{c} \bar{\theta}^{2} \mu\left(K+\bar{t} N^{-1}\right) \leq \bar{c} \bar{\theta}^{2} \mu
$$

Therefore, Lemma 3.4 implies $\mathbb{E}\left[\mathrm{e}^{m_{C}(\bar{t})}\right] \leq \exp \left(\bar{c} \bar{\theta}^{2} \mu\right)$. Thanks to Markov's inequality,

$$
\begin{aligned}
\mathbb{P}\left\{Y_{C}^{N}\left(\bar{t}, \frac{j}{N}\right)>\epsilon_{0}\right\} & =\mathbb{P}\left\{\bar{m}_{C}(\bar{t})>\epsilon_{0}\right\}=\mathbb{P}\left\{m_{C}(\bar{t})>\bar{\theta} \mu \epsilon_{0}\right\} \quad=\mathbb{P}\left\{\mathrm{e}^{m_{C}(\bar{t})}>\mathrm{e}^{\bar{\theta} \mu \epsilon_{0}}\right\} \\
& \leq \mathrm{e}^{-\bar{\theta} \mu \epsilon_{0}} \mathbb{E}\left[\mathrm{e}^{m_{C}(t)}\right] \leq \exp \left[\bar{\theta} \mu\left(\bar{c} \bar{\theta}-\epsilon_{0}\right)\right] .
\end{aligned}
$$

Thus we can choose $\bar{\theta}$ such that

$$
\mathbb{P}\left\{Y_{C}^{N}\left(\bar{t}, \frac{j}{N}\right)>\epsilon_{0}\right\} \leq \mathrm{e}^{-\alpha \epsilon_{0}^{2} \mu}, \text { for } \alpha=\bar{c}\left(\tilde{\rho}_{T}, T\right)>0,
$$

independently of $N, \mu, j$ and $\bar{t}$ (one may solve $\bar{c} \bar{\theta}^{2}-\epsilon_{0} \bar{\theta}+\alpha \epsilon_{0}^{2} \leq 0$ in $\bar{\theta}$ ). The same relation holds for $\mathbb{P}\left\{-Y_{C}^{N}\left(\bar{t}, \frac{j}{N}\right)>\epsilon_{0}\right\}$, by replacing the processes $\bar{m}_{C}$ and $Y_{C}^{N}$ by their opposites $-\bar{m}_{C}$ and $-Y_{C}^{N}$ respectively, and repeating the argument. Therefore,

$$
\mathbb{P}\left\{\left|Y_{C}^{N}\left(t, \frac{j}{N}\right)\right|>\epsilon_{0}\right\} \leq 2 \mathrm{e}^{-\alpha \epsilon_{0}^{2} \mu}, \text { for } 0<t \leq T \text { and } j \in\{1, \cdots, N\} .
$$

Since $\left\|Y_{C}^{N}(t)\right\|_{\infty}=\sup _{1 \leq j \leq N}\left|Y_{C}^{N}\left(t, \frac{j}{N}\right)\right|$ and $Y_{C}^{N}(0)=0$,

$$
\begin{aligned}
\mathbb{P}\left\{\left\|Y_{C}^{N}(t)\right\|_{\infty}>\epsilon_{0}\right\} & =\mathbb{P}\left\{\left|Y_{C}^{N}\left(t, \frac{j}{N}\right)\right|>\epsilon_{0}, \text { for some } j \in\{1, \cdots, N\}\right\} \\
& \leq \sum_{1 \leq j \leq N} \mathbb{P}\left\{\left|Y_{C}^{N}\left(t, \frac{j}{N}\right)\right|>\epsilon_{0}\right\} \leq 2 N \mathrm{e}^{-\alpha \epsilon_{0}^{2} \mu}
\end{aligned}
$$


for $0 \leq t \leq T, \alpha=\alpha(\rho)>0$. We now show that (45) holds with $\left\|Y_{C}^{N}(t)\right\|_{\infty}$ replaced by $\sup _{[0, T]}\left\|Y_{C}^{N}(t)\right\|_{\infty}$ and $N-$ on the r.h.s. - replaced by $N^{3}$. From Duhamel formula, $Y_{C}^{N}(t)=\int_{0}^{t} T_{N}(t-s) d Z_{C}^{N}(s \wedge \tau)$ satisfies the Stochastic Differential Equation (SDE)

$$
d Y_{C}^{N}(t)=\Delta_{N} Y_{C}^{N}(t)+d Z_{C}^{N}(t \wedge \tau)
$$

whose integral form is

$$
Y_{C}^{N}(t)=Z_{C}^{N}(t \wedge \tau)+\int_{0}^{t} \Delta_{N} Y_{C}^{N}(s) d s
$$

We subdivide [0, T] into $N^{2}$ subintervals $I_{n}(T):=\left[\frac{n T}{N^{2}}, \frac{(n+1) T}{N^{2}}\right], 0 \leq n \leq N^{2}-1$. Then taking $t=\frac{n T}{N^{2}}$ in (46) yields

$$
Y_{C}^{N}\left(\frac{n T}{N^{2}}\right)=Z_{C}^{N}\left(\frac{n T}{N^{2}} \wedge \tau\right)+\int_{0}^{\frac{n T}{N^{2}}} \Delta_{N} Y_{C}^{N}(s) d s
$$

One writes

$$
\begin{aligned}
Y_{C}^{N}(t) & =Z_{C}^{N}(t \wedge \tau)+\int_{0}^{\frac{n T}{N^{2}}} \Delta_{N} Y_{C}^{N}(s) d s+\int_{\frac{n T}{N^{2}}}^{t} \Delta_{N} Y_{C}^{N}(s) d s \\
& =Z_{C}^{N}(t \wedge \tau)+\left[Y_{C}^{N}\left(\frac{n T}{N^{2}}\right)-Z_{C}^{N}\left(\frac{n T}{N^{2}} \wedge \tau\right)\right]+\int_{\frac{n T}{N^{2}}}^{t} \Delta_{N} Y_{C}^{N}(s) d s \\
& =Y_{C}^{N}\left(\frac{n T}{N^{2}}\right)+\int_{\frac{n T}{N^{2}}}^{t} \Delta_{N} Y_{C}^{N}(s) d s+\tilde{m}_{C}(t)
\end{aligned}
$$

where

$$
\tilde{m}_{C}(t)=Z_{C}^{N}(t \wedge \tau)-Z_{C}^{N}\left(\frac{n T}{N^{2}} \wedge \tau\right)
$$

defines a mean 0 martingale, for $t \in I_{n}(T)$. Thus,

$$
\left\|Y_{C}^{N}(t)\right\|_{\infty} \leq\left\|Y_{C}^{N}\left(\frac{n T}{N^{2}}\right)\right\|_{\infty}+4 N^{2} \int_{\frac{n T}{N^{2}}}^{t}\left\|Y_{C}^{N}(s)\right\|_{\infty} d s+\left\|\tilde{m}_{C}(t)\right\|_{\infty}
$$

since $\left\|\Delta_{N}\right\|_{\infty} \leq 4 N^{2}$. With the notation $\sup _{t \in I_{n}(T)} \equiv \sup _{I_{n}(T)}$, a Gronwall argument leads to

$$
\sup _{I_{n}(T)}\left\|Y_{C}^{N}(t)\right\|_{\infty} \leq\left(\left\|Y_{C}^{N}\left(\frac{n T}{N^{2}}\right)\right\|_{\infty}+\sup _{I_{n}(T)}\left\|\tilde{m}_{C}(t)\right\|_{\infty}\right) \mathrm{e}^{4 T}
$$

We now want to proceed as previously, by finding a suitable martingale associated with $\tilde{m}_{C}$. Fix $j \in\{1, \cdots, N\}$, $\bar{\theta} \in\left[0,|\gamma|^{-1}\right]$ and set

$$
m_{C}(t)=\bar{\theta} \mu \tilde{m}_{C}\left(t, \frac{j}{N}\right), \text { for } t \in I_{n}(T)=\left[\frac{n T}{N^{2}}, \frac{(n+1) T}{N^{2}}\right] .
$$

For $s \in I_{n}(T), s \geq \frac{n T}{N^{2}}$ yields $\left|\delta m_{C}(s)\right|=\bar{\theta} \mu\left|\delta \tilde{m}_{C}(s)\right|=\bar{\theta} \mu\left|\delta Z_{j}^{N, C}(s \wedge \tau)\right| \leq 1$. Thanks to Lemma 3.2,

$$
\sum_{\frac{n T}{N^{2}} \wedge \tau \leq s \leq t \wedge \tau}\left[\delta m_{C}(s)\right]^{2}-\int_{\frac{n T}{N^{2}} \wedge \tau}^{t \wedge \tau} g_{C}^{N}(s) d s
$$

is a mean 0 martingale for $t \in I_{n}(T)$, where

$$
g_{C}^{N}(s)=\bar{\theta}^{2} \mu\left[N^{2}\left(u_{j-1}^{N, C}(s)+2 u_{j}^{N, C}(s)+u_{j+1}^{N, C}(s)\right)+\left(|F|_{j}^{2}+\mu\left|F_{1}^{N}\right|_{j}^{2}\right)\left(u^{N}(s)\right)\right] .
$$


Now, $0 \leq g_{C}^{N}(s) \leq h_{C}^{N}(s)$ thanks to (38) and the local boundedness of reaction rates, where $h_{C}^{N}(s)=$ $\bar{\theta}^{2} \mu\left(4 N^{2} \tilde{\rho}_{T}+\bar{\gamma}_{C} \bar{\lambda}\left(\tilde{\rho}_{T}\right)\right.$ satisfies $\int_{\frac{n T}{N^{2}} \wedge \tau}^{t \wedge \tau} h_{C}^{N}(s) \leq \bar{c}^{2} \mu T$.

Lemma 3.4 then implies

$$
\mathbb{E}\left[\exp \left\{m_{C}\left(\frac{(n+1) T}{N^{2}}\right)\right\}\right] \leq \exp \left(\bar{c} \bar{\theta}^{2} \mu T\right) .
$$

Applying Doob's inequalities, we have

$$
\begin{aligned}
\mathbb{P}\left\{\sup _{I_{n}(T)} \tilde{m}_{C}\left(t, \frac{j}{N}\right)>\epsilon_{0}\right\} & \leq \mathrm{e}^{-\bar{\theta} \mu \epsilon_{0}} \mathbb{E}\left[\exp \left\{m_{C}\left(\frac{(n+1) T}{N^{2}}\right)\right\}\right] \\
& \leq \exp \left[\bar{\theta} \mu\left(\bar{c} \bar{\theta}-\epsilon_{0}\right)\right] \leq \mathrm{e}^{-\alpha \epsilon_{0}^{2} \mu},
\end{aligned}
$$

where $\alpha=\bar{c}\left(\tilde{\rho}_{T}, T\right)>0$, independently of $N, \mu$ and $j$. We have chosen a suitable $\bar{\theta}$ as previously. Again, the same inequality holds for $-\tilde{m}_{C}\left(t, \frac{j}{N}\right)$ and consequently,

$$
\mathbb{P}\left\{\sup _{I_{n}(T)}\left\|\tilde{m}_{C}(t)\right\|_{\infty}>\epsilon_{0}\right\} \leq 2 N \mathrm{e}^{-\alpha \epsilon_{0}^{2} \mu} .
$$

From (45), (47) and (48),

$$
\begin{aligned}
\mathbb{P}\left\{\mathrm{e}^{-4 T} \sup _{I_{n}(T)}\left\|Y_{C}^{N}(t)\right\|_{\infty}>\epsilon_{0}\right\} & \leq \mathbb{P}\left\{\left\|Y_{C}^{N}\left(\frac{n T}{N^{2}}\right)\right\|_{\infty}+\sup _{I_{n}(T)}\left\|\tilde{m}_{C}(t)\right\|_{\infty}>\epsilon_{0}\right\} \\
& \leq \mathbb{P}\left\{\left\|Y_{C}^{N}\left(\frac{n T}{N^{2}}\right)\right\|_{\infty}>\frac{\epsilon_{0}}{2}\right\}+\mathbb{P}\left\{+\sup _{I_{n}(T)}\left\|\tilde{m}_{C}(t)\right\|_{\infty}>\frac{\epsilon_{0}}{2}\right\} . \\
& \leq 2 N \mathrm{e}^{-\alpha \frac{\epsilon_{0}^{2}}{4} \mu}+2 \mathrm{Ne}^{-\alpha \frac{\epsilon_{0}^{2}}{4} \mu}=4 \mathrm{Ne}^{-\alpha \frac{\epsilon_{0}^{2}}{4} \mu} \leq 4 \mathrm{Ne}^{-\alpha \epsilon_{0}^{2} \mu}
\end{aligned}
$$

Hence,

$$
\mathbb{P}\left\{\mathrm{e}^{-4 T} \sup _{[0, T]}\left\|Y_{C}^{N}(t)\right\|_{\infty}>\epsilon_{0}\right\} \leq \sum_{n=0}^{N^{2}-1} \mathbb{P}\left\{\mathrm{e}^{-4 T} \sup _{I_{n}(T)}\left\|Y_{C}^{N}(t)\right\|_{\infty}>\epsilon_{0}\right\} \leq 4 N^{3} \mathrm{e}^{-\alpha \epsilon_{0}^{2} \mu},
$$

where $\alpha=\alpha\left(\tilde{\rho}_{T}, T\right)>0$. We deduce

$$
\mathbb{P}\left\{\sup _{[0, T]}\left\|Y_{C}^{N}(t)\right\|_{\infty}>\epsilon_{0}\right\} \leq 4 N^{3} \exp \left(-\alpha \mathrm{e}^{-16 T^{2}} \epsilon_{0}^{2} \mu\right) \leq \exp (\log 4+3 \log N-\alpha \mu),
$$

with $\alpha=\alpha\left(\tilde{\rho}_{T}, T, \epsilon_{0}\right)$. The r.h.s. vanishes, since we are assuming $\mu^{-1} \log N \rightarrow 0$. This proves the convergence of the continuous part.

\subsubsection{Martingale discrete component term}

We want to show that

$$
\mathbb{P}\left\{\sup _{[0, T]}\left\|Y_{D}^{N}(t)\right\|_{\infty}>\epsilon_{0}\right\} \rightarrow 0
$$

We use the same procedure as for the continuous part. Fix $\bar{t} \in(0, T]$ and $j \in\{1, \cdots, N\}$. For $0 \leq t \leq \bar{t}$, set

$$
f:=N 1_{j} \text {, and } \bar{m}_{D}(t):=\left\langle Z_{D}^{N}(t \wedge \tau), f\right\rangle_{2} .
$$

Then, the process $\bar{m}_{D}:=\left\{\bar{m}_{D}(t), 0 \leq t \leq \bar{t}\right\}$ defines a mean zero martingale, such that $\bar{m}_{D}(\bar{t})=Y_{D}^{N}\left(\bar{t}, \frac{j}{N}\right)$. Taking $\varphi=f$ in Lemma 3.2 yields

$$
\sum_{s \leq t \wedge \tau}\left[\delta \bar{m}_{D}(s)\right]^{2}-\frac{1}{N} \int_{0}^{t \wedge \tau}\left\langle\left|G^{N}\right|^{2}\left(u^{N}(s)\right), f^{2}\right\rangle_{2} d s
$$


is a mean 0 martingale for $0 \leq t \leq \bar{t}$. From (41),

$$
\left|\delta \bar{m}_{D}(t)\right|=\left|\delta Z_{D}^{N}\left(t \wedge \tau, \frac{j}{N}\right)\right| \leq\left\|\delta Z_{D}^{N}(t \wedge \tau)\right\|_{\infty}=\left\|\delta u_{D}^{N}(t \wedge \tau)\right\|_{\infty} \leq \gamma N^{-1} .
$$

Since $N \rightarrow \infty$, we may assume $N^{-1} \leq 1$. Then, For $\bar{\theta} \in\left[0, \gamma^{-1}\right]$,

$$
m_{D}(t)=\bar{\theta} N \bar{m}_{D}(t)
$$

defines a mean 0 càdlàg martingale such that $\left|\delta m_{D}(t)\right| \leq 1$ and $\left[\delta m_{D}(s)\right]^{2}=\bar{\theta}^{2} N^{2}\left[\delta \bar{m}_{D}(s)\right]^{2}$ for $0 \leq t \leq \bar{t}$. Thus, from Lemma 3.2,

$$
\sum_{s \leq t}\left[\delta m_{D}(s)\right]^{2}-\int_{0}^{t \wedge \tau} g_{D}^{N}(s) d s
$$

is a mean 0 càdlàg martingale, where

$$
\begin{aligned}
g_{D}^{N}(s) & :=\bar{\theta}^{2} N\left\langle\left|G^{N}\right|\left(u^{N}(s)\right), f^{2}\right\rangle_{2}=\bar{\theta}^{2} N^{2}\left|G_{j}^{N}\right|^{2}\left(u^{N}(s)\right) \\
& =\bar{\theta}^{2} N^{2} \sum_{i=1}^{N}\left|\gamma_{i j}^{N}\right|^{2}|g|_{i j}^{2}\left(u^{N}(s)\right) \leq \bar{\theta}^{2} \bar{c} N,
\end{aligned}
$$

thanks to the boundedness of reaction rates. It follows that $0 \leq g_{D}^{N}(s) \leq h_{D}^{N}(s)$, where $h_{D}^{N}(s):=\bar{\theta}^{2} \bar{c} N$ satisfies $\int_{0}^{t} h_{D}^{N}(s) d s \leq \bar{\theta}^{2} \bar{c} N$ for $0 \leq t \leq \bar{t} \leq T$. Therefore, Lemma 3.4 implies $\mathbb{E}\left[\mathrm{e}^{m_{D}(\bar{t})}\right] \leq \exp \left(\bar{\theta}^{2} \bar{c} N\right)$. Markov's inequality yields

$$
\begin{aligned}
\mathbb{P}\left\{Y_{D}^{N}\left(\bar{t}, \frac{j}{N}\right)>\epsilon_{0}\right\} & =\mathbb{P}\left\{\bar{m}_{D}(\bar{t})>\epsilon_{0}\right\}=\mathbb{P}\left\{m_{D}(\bar{t})>\bar{\theta} N \epsilon_{0}\right\}=\mathbb{P}\left\{\mathrm{e}^{m_{D}(\bar{t})}>\mathrm{e}^{\bar{\theta} N \epsilon_{0}}\right\} \\
& \leq \mathrm{e}^{-\bar{\theta} N \epsilon_{0}} \mathbb{E}\left[\mathrm{e}^{m_{D}(t)}\right] \leq \exp \left[\bar{\theta} N\left(\bar{c} \bar{\theta}-\epsilon_{0}\right)\right] . \\
\mathbb{P}\left\{Y_{D}^{N}\left(\bar{t}, \frac{j}{N}\right)>\epsilon_{0}\right\} & =\mathbb{P}\left\{\bar{m}_{D}(\bar{t})>\epsilon_{0}\right\}=\mathbb{P}\left\{m_{D}(\bar{t})>\bar{\theta} N \epsilon_{0}\right\}=\mathbb{P}\left\{\mathrm{e}^{m_{D}(\bar{t})}>\mathrm{e}^{\bar{\theta} N \epsilon_{0}}\right\} \\
& \leq \mathrm{e}^{-\bar{\theta} N \epsilon_{0}} \mathbb{E}\left[\mathrm{e}^{m_{D}(t)}\right] \leq \exp \left[\bar{\theta} N\left(\bar{c} \bar{\theta}-\epsilon_{0}\right)\right] .
\end{aligned}
$$

We can choose $\bar{\theta}$ such that

$$
\mathbb{P}\left\{Y_{D}^{N}\left(\bar{t}, \frac{j}{N}\right)>\epsilon_{0}\right\} \leq \mathrm{e}^{-\alpha \epsilon_{0}^{2} N}, \text { for } \alpha=\bar{c}\left(M_{1}, \rho, T\right)>0,
$$

independently of $N, \mu, j$ and $\bar{t}$. The same relation holds for $\mathbb{P}\left\{-Y_{D}^{N}\left(\bar{t}, \frac{j}{N}\right)>\epsilon_{0}\right\}$. Therefore,

$$
\mathbb{P}\left\{\left|Y_{D}^{N}\left(t, \frac{j}{N}\right)\right|>\epsilon_{0}\right\} \leq 2 \mathrm{e}^{-\alpha \epsilon_{0}^{2} N} \text { for } 0<t \leq T \text { and } j \in 1, \ldots, N
$$

Since $\left\|Y_{D}^{N}(t)\right\|_{\infty}=\sup _{1 \leq j \leq N}\left|Y_{D}^{N}\left(t, \frac{j}{N}\right)\right|$ and $Y_{D}^{N}(0)=0$

$$
\begin{aligned}
\mathbb{P}\left\{\left\|Y_{D}^{N}(t)\right\|_{\infty}>\epsilon_{0}\right\} & =\mathbb{P}\left\{\left|Y_{D}^{N}\left(t, \frac{j}{N}\right)\right|>\epsilon_{0}, \text { for some } j \in\{1, \cdots, N\}\right\} \\
& \leq \sum_{1 \leq j \leq N} \mathbb{P}\left\{\left|Y_{D}^{N}\left(t, \frac{j}{N}\right)\right|>\epsilon_{0}\right\} \leq 2 N \mathrm{e}^{-\alpha \epsilon_{0}^{2} N}
\end{aligned}
$$

for $0 \leq t \leq T$. We now show that (51) holds with $\left\|Y_{D}^{N}(t)\right\|_{\infty}$ replaced by $\sup _{[0, T]}\left\|Y_{D}^{N}(t)\right\|_{\infty}$ and $N$ (on the right) replaced by $N^{3}$. With the introduced subdivision of $[0, T]$ into $N^{2}$ subintervals $I_{n}(T)=\left[\frac{n T}{N^{2}}, \frac{(n+1) T}{N^{2}}\right], 0 \leq n \leq$ $N^{2}-1$, one can always write

$$
Y_{D}^{N}(t)=\tilde{m}_{D}(t)+Z_{D}^{N}\left(\frac{n T}{N^{2}} \wedge \tau\right)=\tilde{m}_{D}(t)+Y_{D}^{N}\left(\frac{n T}{N^{2}}\right)
$$


where $\tilde{m}_{D}(t):=Z_{D}^{N}(t \wedge \tau)-Z_{D}^{N}\left(\frac{n T}{N^{2}} \wedge \tau\right)$ is a mean zero martingale for $t \in I_{n}(T)$. Thus,

$$
\sup _{I_{n}(T)}\left\|Y_{D}^{N}(t)\right\|_{\infty} \leq \sup _{I_{n}(T)}\left\|\tilde{m}_{D}(t)\right\|_{\infty}+\left\|Y_{D}^{N}\left(\frac{n T}{N^{2}}\right)\right\|_{\infty} .
$$

As previously, we now want to find a suitable martingale associated with $\tilde{m}_{D}$. Fix $j \in\{1, \cdots, N\}, \bar{\theta} \in\left[0, \gamma^{-1}\right]$ and set

$$
m_{D}(t)=\bar{\theta} N \tilde{m}_{D}\left(t, \frac{j}{N}\right), \text { for } t \in I_{n}(T)=\left[\frac{n T}{N^{2}}, \frac{(n+1) T}{N^{2}}\right] .
$$

For $s \in I_{n}(T), s \geq \frac{n T}{N^{2}}$ yields $\left|\delta m_{D}(s)\right|=\bar{\theta} N\left|\delta Z_{j}^{N, D}(s \wedge \tau)\right| \leq 1$. From Lemma 3.2,

$$
\sum_{\frac{n T}{N^{2}} \leq s \leq t \wedge \tau}\left[\delta m_{D}(s)\right]^{2}-\int_{\frac{n T}{N^{2}} \wedge \tau}^{t \wedge \tau} g_{D}^{N}(s) d s
$$

is a mean 0 martingale, with $g_{D}^{N}(s)=\bar{\theta}^{2} N^{2}\left|G^{N}\right|_{j}^{2}\left(u^{N}(s)\right) \leq \bar{\theta}^{2} \bar{c} N$. Since reaction rates are bounded, $0 \leq g_{D}^{N}(s) \leq h_{D}^{N}(s)$, where $h_{D}^{N}(s)=\bar{\theta}^{2} \bar{c} N$ satisfies $\int_{\frac{n T}{N^{2}} \wedge \tau}^{t \wedge \tau} h_{D}^{N}(s) \leq \bar{c} \bar{\theta}^{2} N$. Thus, Lemma 3.4 implies $\mathbb{E}\left[\exp \left\{m_{D}\left(\frac{(n+1) T}{N^{2}}\right)\right\}\right] \leq \exp \left(\bar{c}^{2} N\right)$. Doob's inequalities, lead to

$$
\begin{aligned}
\mathbb{P}\left\{\sup _{I_{n}(T)} \tilde{m}_{D}\left(t, \frac{j}{N}\right)>\epsilon_{0}\right\} & \leq \mathrm{e}^{-\bar{\theta} N \epsilon_{0}} \mathbb{E}\left[\exp \left\{m_{D}\left(\frac{(n+1) T}{N^{2}}\right)\right\}\right], \\
& \leq \exp \left[\bar{\theta} N\left(\bar{c} \bar{\theta}-\epsilon_{0}\right)\right] \leq \mathrm{e}^{-\alpha \epsilon_{0}^{2} N}
\end{aligned}
$$

where $\alpha=\bar{c}\left(\tilde{\rho}_{T}, T\right)>0$, independently of $N, \mu$ and $j$. We have chosen a suitable $\bar{\theta}$ as previously. Also, the same holds for $-\tilde{m}_{D}\left(t, \frac{j}{N}\right)$. This shows that

$$
\mathbb{P}\left\{\sup _{I_{n}(T)}\left\|\tilde{m}_{D}(t)\right\|_{\infty}>\epsilon_{0}\right\} \leq 2 N \mathrm{e}^{-\alpha \epsilon_{0}^{2} N} .
$$

From (51),(52) and (53), we have

$$
\begin{aligned}
\mathbb{P}\left\{\mathrm{e}^{-4 T} \sup _{I_{n}(T)}\left\|Y_{D}^{N}(t)\right\|_{\infty}>\epsilon_{0}\right\} & \leq \mathbb{P}\left\{\left\|Y_{D}^{N}\left(\frac{n T}{N^{2}}\right)\right\|_{\infty}+\sup _{I_{n}(T)}\left\|\tilde{m}_{D}(t)\right\|_{\infty}>\epsilon_{0}\right\} \\
& \leq \mathbb{P}\left\{\left\|Y_{D}^{N}\left(\frac{n T}{N^{2}}\right)\right\|_{\infty}>\frac{\epsilon_{0}}{2}\right\}+\mathbb{P}\left\{+\sup _{I_{n}(T)}\left\|\tilde{m}_{D}(t)\right\|_{\infty}>\frac{\epsilon_{0}}{2}\right\} \\
& \leq 2 N \mathrm{e}^{-\alpha \frac{\epsilon_{0}^{2}}{2} N}+2 \mathrm{Ne}^{-\alpha \frac{\epsilon_{0}^{2}}{2} N} \leq 4 N \mathrm{e}^{-\alpha \epsilon_{0}^{2} N} .
\end{aligned}
$$

Hence,

$$
\mathbb{P}\left\{\mathrm{e}^{-4 T} \sup _{[0, T]}\left\|Y_{D}^{N}(t)\right\|_{\infty}>\epsilon_{0}\right\} \leq \sum_{n=0}^{N^{2}-1} \mathbb{P}\left\{\mathrm{e}^{-4 T} \sup _{I_{n}(T)}\left\|Y_{D}^{N}(t)\right\|_{\infty}>\epsilon_{0}\right\} \leq 4 N^{3} \mathrm{e}^{-\alpha \epsilon_{0}^{2} N} .
$$

Setting $c:=\alpha \mathrm{e}^{-4 T} \epsilon_{0}>0$, it follows that

$$
\mathbb{P}\left\{\sup _{[0, T]}\left\|Y_{D}^{N}(t)\right\|_{\infty}>\epsilon_{0}\right\} \leq 4 N^{3} \mathrm{e}^{-c N} \rightarrow 0 \text { as } N \rightarrow \infty,
$$

which shows the convergence for the discrete part. The proofs of (44) and thus of Theorem 2.2 are complete.

\section{Remark 3.3}

In this proof we have used in a crucial way that at each point, the jump of the discrete component is of order $\frac{1}{N}$. For the original model, without spatial correlation of the slow reactions, this does not hold since the jump of discrete components are of order 1. Clearly, we could slightly change our assumption and assume that slow reactions affects neighboring sites at distance of order $N^{-} \alpha, \alpha \in[0,1)$, whereas we have considered only $\alpha=0$ above. With this new setting, the discrete jump rates would be of order $\frac{1}{N^{1-\alpha}}$ and our conclusion would still hold. 


\section{A Appendix}

\section{A.1 Formal limit of the generator}

We consider the sequence $\mathscr{A}^{N}=\mathscr{A}^{N, \mu}$ of generators defined by (13). We consider a $L^{2}(I)$ framework and consider test functions $\varphi \in C_{b}^{2}\left(L^{2}(I) \times L^{2}(I)\right)$. Each line is expanded at order two thanks to Taylor expansion. The first order expansion has already been examined to understand the limiting generator. For $k=1, \cdots, 5$, denote by $T_{k}(N)$ the second order term corresponding to the $k$-th line in the expression of the generator. Let $u=\left(u_{C}, u_{D}\right) \in C_{p}(I) \times C_{p}(I)$ be fixed and let $N, \mu \rightarrow \infty$.

We assume that reaction rates are bounded.

At order 1. We already know that the whole terms together read

$$
\begin{aligned}
\mathscr{A}_{1}^{N} \varphi\left(\tilde{P}_{N} u\right)= & \left\langle D^{1,0} \varphi\left(\tilde{P}_{N} u\right), \Delta_{N} P_{N} u_{C}+F\left(\tilde{P}_{N} u\right)+F_{1}^{N}\left(\tilde{P}_{N} u\right)\right\rangle_{2} \\
& +\left\langle D^{0,1} \varphi\left(\tilde{P}_{N} u\right), G^{N}\left(\tilde{P}_{N} u\right)\right\rangle_{2} .
\end{aligned}
$$

For $u_{C} \in C^{3}(I)$, it converges to

$$
\mathscr{A}^{\infty} \varphi(u)=\left\langle D^{1,0} \varphi(u), \Delta u_{C}+F(u)\right\rangle_{2}+\left\langle D^{0,1} \varphi(u), G(u)\right\rangle_{2},
$$

requiring $G^{N}\left(\tilde{P}_{N} u\right) \rightarrow G(u)$, which follows from Proposition 2.1, Proposition 3.1 and

$$
\begin{aligned}
\left\|G^{N}\left(\tilde{P}_{N} u\right)-G(u)\right\|_{\infty} & \leq\left\|G^{N}\left(\tilde{P}_{N} u\right)-G^{N}(u)\right\|_{\infty}+\left\|G^{N}(u)-G(u)\right\|_{\infty} \\
& \leq L_{0}\left\|P_{N} u-u\right\|_{\infty, \infty}+\left\|G^{N}(u)-G(u)\right\|_{\infty},
\end{aligned}
$$

where we use the same notation as in appendix A.4.

At order 2. We introduce $e_{j}:=\sqrt{N} \mathbb{1}_{j}$, for $1 \leq j \leq N$. Since the functions $\left(\mathbb{1}_{j}\right)_{1 \leq j \leq N}$ are pairwise orthogonal in $L^{2}$ and of norm $\left\|\mathbb{1}_{j}\right\|_{2}=\frac{1}{\sqrt{N}}$, the family $\left\{e_{j}, 1 \leq j \leq N\right\}$ forms an orthonormal basis of $\left(\mathbb{\mathbb { A } ^ { N }},\|\cdot\|_{2}\right)$. Also, $c$ denotes a generic constant and $\left\|D^{l, k} \varphi\right\|_{\infty}$ denotes a uniform bound of $D^{l, k} \varphi$, for $0 \leq l, k \leq 2, l+k \leq 2$. Let us treat the term appearing from the second line,

$$
T_{2}(N)=\frac{1}{2} \sum_{r \in S_{1}} \sum_{j=1}^{N} D^{2,0} \varphi\left(\tilde{P}_{N} u\right) \cdot\left\langle\frac{\gamma_{r}^{C}}{\mu} \mathbb{1}_{j}, \frac{\gamma_{r}^{C}}{\mu} \mathbb{1}_{j}\right\rangle_{2} \mu \lambda_{r}\left(u_{j}^{C}, u_{j}^{D}\right) \leq \frac{\bar{\gamma}^{2} \bar{\lambda}}{2}\left\|D^{2,0} \varphi\right\|_{\infty} \frac{1}{N \mu} \sum_{j=1}^{N}\left\|e_{j}\right\|_{2}^{2} \leq \frac{c}{\mu} \longrightarrow 0 .
$$

The first term is similar and also vanishes at the limit, using the same argument. Next, the third term reads

$$
\begin{aligned}
T_{3}(N)= & \left.\frac{1}{2} \sum_{r \in \Re_{D C} \backslash S_{1}} \sum_{j=1}^{N} D^{2,0} \varphi\left(\tilde{P}_{N} u\right) \cdot\left\langle\frac{\gamma_{r}^{C}}{\mu} \mathbb{1}_{j}, \frac{\gamma_{r}^{C}}{\mu} \mathbb{1}_{j}\right)\right\rangle_{2} \lambda_{r}\left(u_{j}\right) \\
& +\frac{1}{2} \sum_{r \in \Re_{D C} \backslash S_{1}} \sum_{j=1}^{N} D^{1,1} \varphi\left(\tilde{P}_{N} u\right) \cdot\left\langle\frac{\gamma_{r}^{C}}{\mu} \mathbb{1}_{j}, \gamma_{j, r}^{N, D}\left(\tilde{P}_{N} u\right)\right\rangle_{2} \lambda_{r}\left(u_{j}\right) \\
& +\frac{1}{2} \sum_{r \in \mathfrak{R}_{D C} \backslash S_{1}} \sum_{j=1}^{N} D^{0,2} \varphi\left(\tilde{P}_{N} u\right) \cdot\left\langle\gamma_{j, r}^{N, D}\left(P_{N} u_{D}\right), \gamma_{j, r}^{N, D}\left(\tilde{P}_{N} u\right)\right\rangle_{2} \lambda_{r}\left(u_{j}\right) .
\end{aligned}
$$

We have

$$
\begin{aligned}
T_{31}(N) & :=\frac{1}{2 N} \sum_{r \in \Re_{D C} \backslash S_{1}} \sum_{j=1}^{N} D^{2,0} \varphi\left(\tilde{P}_{N} u\right) \cdot\left\langle\frac{\gamma_{r}^{C}}{\mu} e_{j}, \frac{\gamma_{r}^{C}}{\mu} e_{j}\right\rangle_{2} \lambda_{r}\left(u_{j}\right) \\
& c\left\|D^{2,0} \varphi\right\|_{\infty} \frac{1}{N \mu^{2}} \sum_{j=1}^{N} e_{j}^{2} \frac{c}{\mu^{2}} \rightarrow 0
\end{aligned}
$$

Observing that

$$
\left\|\gamma_{j, r}^{N, D}\left(\tilde{P}_{N} u\right)\right\|_{2} \leq\left|\gamma_{r}^{D}\right| \sum_{i=1}^{N}\left|\gamma_{i j}(N)\right|\left\|\mathbb{1}_{i}\right\|_{2} \leq\left|\gamma_{r}^{D}\right| \frac{a(0)}{\sqrt{N}}
$$


for all $1 \leq j \leq N$, we deduce from the inequality of Cauchy-Schwarz

$$
\begin{aligned}
T_{32}(N) & :=\frac{1}{2 \sqrt{N}} \sum_{r \in \mathfrak{R}_{D C} \backslash S_{1}} \sum_{j=1}^{N} D^{1,1} \varphi\left(\tilde{P}_{N} u\right) \cdot\left\langle\frac{\gamma_{r}^{C}}{\mu} e_{j}, \gamma_{j, r}^{N, D}\left(\tilde{P}_{N} u\right)\right\rangle_{2} \lambda_{r}\left(u_{j}\right) \\
& \leq c\left\|D^{1,1} \varphi\right\|_{\infty} \frac{1}{\sqrt{N} \mu} \sum_{j=1}^{N}\left\|e_{j}\right\|_{2}\left\|\gamma_{j, r}^{N, D}\left(\tilde{P}_{N} u\right)\right\|_{2} \leq \frac{c}{\sqrt{N} \mu} \longrightarrow 0,
\end{aligned}
$$

on the one hand, and, on the other hand

$$
T_{33}(N):=\frac{1}{2} \sum_{r \in \mathfrak{R}_{D C} \backslash S_{1}} \sum_{j=1}^{N} D^{0,2} \varphi\left(\tilde{P}_{N} u\right) \cdot\left\langle\gamma_{j, r}^{N, D}\left(\tilde{P}_{N} u\right), \gamma_{j, r}^{N, D}\left(\tilde{P}_{N} u\right)\right\rangle_{2} \lambda_{r}\left(u_{j}\right)
$$

Recall that, setting $\tilde{P}_{N} u=u^{N}$,

$$
\gamma_{j, r}^{N, D}\left(u_{C}^{N}, u_{D}^{N}\right):=\gamma_{r}^{D} \sum_{i=1}^{N} \gamma_{i j}^{N} \mathbb{1}_{i} \theta_{i j}^{r}\left(u_{i}^{N, C}, u_{i}^{N, D}\right),
$$

where $\gamma_{i j}^{N}:=\int_{I_{i}} a\left(x-\frac{j}{N}\right) d x$, and $\theta_{i j}^{r}\left(u_{i}^{N, C}, u_{i}^{N, D}\right)=\theta\left(u_{i}^{N, C}+\frac{\gamma_{r}^{C}}{\mu} \gamma_{i j}^{N}\right) \theta\left(u_{i}^{N, D}+\gamma_{r}^{D} \gamma_{i j}^{N}\right)$. Define the operator $A_{r}$ by:

$$
A_{r} e_{j}=\sqrt{N} \sqrt{\lambda_{r}\left(u_{j}^{N}\right)} \gamma_{j, r}^{N, D}=\gamma_{r}^{D} \sum_{i=1}^{N} \gamma_{i j}^{N} \theta_{i j}^{r}\left(u_{i}^{N}\right) \sqrt{\lambda_{r}\left(u_{j}^{N}\right)} e_{i}
$$

Then, using standard property of the trace,

$$
\begin{aligned}
T_{33}(N) & =\frac{1}{2 N} \sum_{r \in \Re_{D C} \backslash S_{1}} \sum_{j=1}^{N} D^{0,2} \varphi\left(u^{N}\right) \cdot\left\langle A_{r} e_{j}, A_{r} e_{j}\right\rangle_{2} \\
& =\frac{1}{2 N} \sum_{r \in \Re_{D C} \backslash S_{1}} \operatorname{Tr}\left(A_{r}^{*} D^{0,2} \varphi\left(u^{N}\right) A_{r}\right) \\
& \leq \frac{1}{2 N}\left\|D^{0,2} \varphi\left(u^{N}\right)\right\|_{\infty} \sum_{r \in \mathfrak{R}_{D C} \backslash S_{1}} \operatorname{Tr}\left(A_{r}^{*} A_{r}\right) .
\end{aligned}
$$

Also

$$
\operatorname{Tr}\left(A_{r}^{*} A_{r}\right)=\sum_{j=1}^{N}\left\|A_{r} e_{j}\right\|_{2}^{2} \leq \bar{\lambda}\left(\tilde{\rho}_{T}\right) \bar{\gamma}^{2} \sum_{i, j}^{N}\left|\gamma_{i j}^{N}\right|^{2}
$$

and since $\left|\gamma_{i j}^{N}\right|^{2}=\int_{I_{i}} a\left(x-\frac{j}{N}\right) d x=\frac{1}{\sqrt{N}}\left\langle a\left(\cdot-\frac{j}{N}\right), e_{i}\right\rangle_{2}^{2}$ we have thanks to Parseval identity:

$$
\sum_{i}^{N}\left|\gamma_{i j}^{N}\right|^{2}=\frac{1}{N}\left\|a\left(\cdot-\frac{j}{N}\right)\right\|_{2}^{2}=\frac{1}{N}\|a\|_{2}^{2}
$$

It follows that

$$
\operatorname{Tr}\left(A_{r}^{*} A_{r}\right) \leq \bar{\lambda} \bar{\gamma}^{2}\|a\|_{2}^{2}
$$

and as a result, $T_{3}(N) \longrightarrow 0$. The fourth term also vanishes at the limit, using the same argument.

\section{Remark A.1}

Again, we see why we had to consider some spatial correlation in our model. Indeed, the case without spatial correlation for the discrete species corresponds to $\gamma_{i j}^{N}=1$ for $i=j$ and is 0 otherwise, obtained with a being the Dirac mass. In this case $\operatorname{Tr}\left(A_{r}^{*} A_{r}\right)$ is of order $N$ and $T_{3}(N)$ does not converge to 0 .

Let us consider the fifth. From

$$
\left\|\mathbb{1}_{j+1}-\mathbb{1}_{j}\right\|_{2}^{2}=\frac{2}{N}=\left\|\mathbb{1}_{j-1}-\mathbb{1}_{j}\right\|_{2}^{2}
$$


we have

$$
\begin{aligned}
T_{5}(N)= & \frac{1}{2} \sum_{j=1}^{N}\left[D^{2,0} \varphi\left(\tilde{P}_{N} u\right) \cdot\left\langle\frac{\mathbb{1}_{j+1}-\mathbb{1}_{j}}{\mu}, \frac{\mathbb{1}_{j+1}-\mathbb{1}_{j}}{\mu}\right\rangle_{2}\right. \\
& \left.+D^{2,0} \varphi\left(\tilde{P}_{N} u\right) \cdot\left\langle\frac{\mathbb{1}_{j-1}-\mathbb{1}_{j}}{\mu}, \frac{\mathbb{1}_{j-1}-\mathbb{1}_{j}}{\mu}\right\rangle_{2}\right] \mu N^{2} u_{j}^{C} \\
\leq & u_{C} \infty 2\left\|D^{2} \varphi\right\|_{\infty} \frac{N^{2}}{\mu} \sum_{j=1}^{N}\left(\mathbb{1}_{j+1}-\mathbb{1}_{j_{2}}^{2}+\mathbb{1}_{j-1}-\mathbb{1}_{j_{2}}^{2}\right) \\
\leq & c \mu^{-1} N^{2} \rightarrow 0,
\end{aligned}
$$

if $\mu^{-1} N^{2} \rightarrow 0$ as $N, \mu \rightarrow \infty$.

Hence, under the additional condition $\mu^{-1} N^{2} \rightarrow 0$, all the terms of the second order in the Taylor expansion vanish at the limit and hence, $\mathscr{A}^{\infty}$ is indeed formally the limit of $\mathscr{A}^{N}$.

\section{A.2 Relation between the limit and its discretization}

Proof of Theorem 2.1. Let $T>0$ be fixed. Since the operator $\tilde{\Delta}_{N}$ is linear, it is Lipschitz. Next, the vector field $R=(F, G)$ is locally Lipschitz continuous. Therefore, the initial value problem (26) has a unique local solution $v^{N}$ thanks to the Picard-Lindelöf theorem. The bounds (28) are proved thanks to the discrete maximum principle and Gronwall lemma as in Section 3.2 and we deduce that $v^{N}$ is in fact a global solution.

Thanks to (28) and (25), we may assume that $F$ and $G$ are globally Lipschitz and we choose $L$ such that

$$
\begin{aligned}
& \left\|F\left(u_{C}^{1}, u_{D}^{1}\right)-F\left(u_{C}^{2}, u_{D}^{2}\right)\right\|_{\infty} \leq L\left\|\left(u_{C}^{1}, u_{D}^{1}\right)-\left(u_{C}^{2}, u_{D}^{2}\right)\right\|_{\infty, \infty}, \\
& \left\|G\left(u_{C}^{1}, u_{D}^{1}\right)-G\left(u_{C}^{2}, u_{D}^{2}\right)\right\|_{\infty} \leq L\left\|\left(u_{C}^{1}, u_{D}^{1}\right)-\left(u_{C}^{2}, u_{D}^{2}\right)\right\|_{\infty, \infty},
\end{aligned}
$$

provided $\left\|u_{C}\right\|_{\infty} \leq \rho_{C},\left\|u_{D}\right\|_{\infty} \leq\left(\rho_{D}+1\right) \mathrm{e}^{a(0) M_{1}\left(\rho_{C}\right) T}$.

we have

$$
\begin{aligned}
v^{N}(t)-u(t)= & \tilde{T}_{N}(t) \tilde{P}_{N} u(0)-\tilde{T}(t) u(0) \\
& +\int_{0}^{t} \tilde{T}_{N}(t-s)\left(\begin{array}{c}
F\left(v^{N}(s)\right) \\
G\left(v^{N}(s)\right)
\end{array}\right)-\tilde{T}(t-s)\left(\begin{array}{l}
F(u(s)) \\
G(u(s))
\end{array}\right) d s .
\end{aligned}
$$

for all $t \geq 0$. Then,

$$
\begin{aligned}
\left\|v^{N}(t)-u(t)\right\|_{\infty, \infty} \leq & \left\|\tilde{T}_{N}(t) \tilde{P}_{N} u(0)-\tilde{T}(t) u(0)\right\|_{\infty, \infty} \\
& +\int_{0}^{t}\left\|\tilde{T}_{N}(t-s)\left(\left(\begin{array}{c}
F\left(v^{N}(s)\right) \\
G\left(v^{N}(s)\right)
\end{array}\right)-\tilde{P}_{N}\left(\begin{array}{l}
F(v(s)) \\
G(v(s))
\end{array}\right)\right)\right\|_{\infty, \infty} d s \\
& +\int_{0}^{t}\left\|\tilde{T}_{N}(t-s) \tilde{P}_{N}\left(\begin{array}{l}
F(u(s)) \\
G(u(s))
\end{array}\right)-\tilde{T}(t-s)\left(\begin{array}{l}
F(u(s)) \\
G(u(s))
\end{array}\right)\right\|_{\infty, \infty} d s .
\end{aligned}
$$

Since $\mathbb{U}^{N}$ is stable by $F$ and $G$, we rely on Proposition 2.1 and Proposition 2.2 to find an upper bound for each term on the r.h.s. of the inequality above.

$$
T_{2}(N) \leq \int_{0}^{t}\left\|\tilde{P}_{N}\left(\begin{array}{c}
F\left(v^{N}(s)\right)-F(v(s)) \\
G\left(v^{N}(s)\right)-G(v(s))
\end{array}\right)\right\|_{\infty, \infty} d s \leq 2 L \int_{0}^{t}\left\|v^{N}(s)-v(s)\right\|_{\infty, \infty} d s,
$$

and $T_{3}(N)=\int_{0}^{t}\left\|T_{N}(t-s) P_{N} F(u(s))-T(t-s) F(u(s))\right\|_{\infty} d s$.

Hence,

$$
\begin{aligned}
\left\|v^{N}(t)-v(t)\right\|_{\infty, \infty} \leq & \left\|\tilde{T}_{N}(t) \tilde{P}_{N} v(0)-\tilde{T}(t) v(0)\right\|_{\infty, \infty} \\
& +\int_{0}^{t}\left\|T_{N}(t-s) P_{N} F(v(s))-T(t-s) F(v(s))\right\|_{\infty} d s \\
& +2 L \int_{0}^{t}\left\|v^{N}(s)-v(s)\right\|_{\infty, \infty} d s .
\end{aligned}
$$


Taking the supremum in $t$ on $[0, T]$ and using Gronwall lemma leads to

$$
\begin{aligned}
\sup _{[0, T]}\left\|v^{N}(t)-v(t)\right\|_{\infty, \infty} \leq & \left(\sup _{[0, T]}\left\|\tilde{T}_{N}(t) \tilde{P}_{N} v(0)-\tilde{T}(t) v(0)\right\|_{\infty, \infty}\right. \\
& \left.+\int_{0}^{T} \sup _{t \in[0, T]}\left(\left\|T_{N}(t-s) P_{N} F(v(s))-T(t-s) F(v(s))\right\|_{\infty} \mathbb{1}_{(s \leq t)}\right) d s\right) \times \mathrm{e}^{2 L T} .
\end{aligned}
$$

Firstly,

$$
\begin{aligned}
& \sup _{[0, T]}\left\|\tilde{T}_{N}(t) \tilde{P}_{N} v(0)-\tilde{T}(t) v(0)\right\|_{\infty, \infty} \\
& \quad \leq \sup _{[0, T]}\left\|T_{N}(t) P_{N} u_{C}(0)-T(t) u_{C}(0)\right\|_{\infty}+\left\|P_{N} u_{D}(0)-u_{D}(0)\right\|_{\infty} \rightarrow 0,
\end{aligned}
$$

where the first term vanishes thanks to Proposition 2.2 (vi), since $u_{C}(0) \in C^{3}(I)$. Secondly, we fix $t \in[0, T]$ and $s \in[0, t]$. Since $F(u(s)) \in C^{3}(I)$, we have

$$
\left\|T_{N}(t-s) P_{N} F(u(s))-T(t-s) F(u(s))\right\|_{\infty} \rightarrow 0,
$$

thanks to Proposition 2.2 (vi) again. Moreover, the convergence is uniform w.r.t. $t$, on $[0, T]$. Thus,

$$
\sup _{t \in[0, T]}\left(\left\|T_{N}(t-s) P_{N} F(u(s))-T(t-s) F(u(s))\right\|_{\infty} \mathbb{1}_{(s \leq t)}\right) \rightarrow 0 .
$$

Hence, $\int_{0}^{T} \sup _{t \in[0, T]}\left(\left\|T_{N}(t-s) P_{N} F(u(s))-T(t-s) F(u(s))\right\|_{\infty} \mathbb{1}_{(s \leq t)}\right) d s \rightarrow 0$,

thanks to the dominated convergence theorem.

\section{A.3 The accompanying martingales}

Proof of Lemma 3.1 .

Fix $1 \leq j \leq N$ and define $Y^{N}(t)=\left(Y_{1}^{N}(t), \cdots, Y_{N+4}^{N}(t)\right)$ by

$$
\left\{\begin{array}{l}
Y_{k}^{N}(t):=u_{k}^{N}(t) \text { for } 1 \leq k \leq N \\
Y_{N+1}^{N}(t):=\sum_{s \leq t}\left[\delta u_{j-1}^{N, C}(s)\right]\left[\delta u_{j}^{N, C}(s)\right] \\
Y_{N+2}^{N}(t):=\sum_{s \leq t}\left[\delta u_{j}^{N, C}(s)\right]^{2} \\
Y_{N+3}^{N}(t):=\sum_{s \leq t}\left[\delta u_{j}^{N, C}(s)\right]\left[\delta u_{j+1}^{N, C}(s)\right] \\
Y_{N+4}^{N}(t):=\sum_{s \leq t}\left[\delta u_{j}^{N, D}(s)\right]^{2}
\end{array}\right.
$$

$Y^{N}$ is a Markov process with values in $\mathbb{R}^{2 N+4}$. From (9), Assumptions 2.3 and 2.4, we know that $Y^{N}$ enjoys the following transitions: $Y_{N+1}^{N} \rightarrow Y_{N+1}^{N}-\frac{1}{\mu^{2}}$ at rate $\mu N^{2}\left(u_{j-1}^{N, C}+u_{j}^{N, C}\right)$ so that the corresponding debit is

$$
-\frac{N^{2}}{\mu}\left(u_{j-1}^{C}+u_{j}^{C}\right)=:\left|\Psi_{-}^{N, C}\right|_{j}^{2}(u) .
$$

Similarly, $Y_{N+3}^{N} \rightarrow Y_{N+3}^{N}-\frac{1}{\mu^{2}}$ at rate $\mu N^{2}\left(u_{j}^{N, C}+u_{j+1}^{N, C}\right)$, and the associated debit is

$$
-\frac{N^{2}}{\mu}\left(u_{j}^{C}+u_{j+1}^{C}\right)=:\left|\Psi_{+}^{N, C}\right|^{2}\left(u_{j}\right)
$$

Now,

$$
\begin{array}{lr}
Y_{N+2}^{N} \rightarrow Y_{N+2}^{N}+\frac{\left|\gamma_{r}^{C}\right|^{2}}{\mu^{2}} & \text { at rate }\left\{\begin{array}{l}
\mu \lambda_{r}\left(u_{j}^{N, C}\right) \text { for } r \in \Re_{C} \\
\mu \lambda_{r}\left(u_{j}^{N, C}, u_{j}^{N, D}\right) \text { for } r \in S_{1},
\end{array}\right. \\
Y_{N+2}^{N} \rightarrow Y_{N+2}^{N}+\frac{\left|\gamma_{r}^{C}\right|^{2}}{\mu^{2}} & \text { at rate } \lambda_{r}\left(u_{j}^{N, C}, u_{j}^{N, D}\right) \text { for } r \in \Re_{D C \backslash S_{1}} \\
Y_{N+2}^{N} \rightarrow Y_{N+2}^{N}+\frac{1}{\mu^{2}} & \text { at rate } \mu N^{2}\left(u_{j-1}^{N, C}+2 u_{j}^{N, C}+u_{j+1}^{N, C}\right) \text { for a diffusion. }
\end{array}
$$


The joined debit is

$$
\begin{aligned}
& \frac{1}{\mu}\left[N^{2}\left(u_{j-1}^{C}+2 u_{j}^{C}+u_{j+1}^{C}\right)+\sum_{r \in \Re_{C}}\left|\gamma_{r}^{C}\right|^{2} \lambda_{r}\left(u_{j}^{C}\right)+\sum_{r \in S_{1}}\left|\gamma_{r}^{C}\right|^{2} \lambda_{r}\left(u_{j}^{C}, u_{j}^{D}\right)\right. \\
& \left.\quad+\frac{1}{\mu} \sum_{i=1}^{N} \sum_{r \in \Re_{D C} \backslash S_{1}}\left|\gamma_{j i}^{N}\right|^{2}\left|\theta_{j i}^{r}\left(u_{j}^{C}, u_{j}^{D}\right)\right|^{2} \lambda_{r}\left(u_{j}^{C}, u_{j}^{D}\right)\right] \\
& =\frac{1}{\mu}\left(\left|\Delta_{N}\right|^{2}\left(u_{j}^{C}\right)+|F|_{j}^{2}\left(u_{C}, u_{D}\right)+\left|F_{1}^{N}\right|_{j}^{2}\left(u_{C}, u_{D}\right)\right)=\frac{1}{\mu}\left|\Psi_{C}^{N}\right|_{j}^{2}\left(u_{C}, u_{D}\right) .
\end{aligned}
$$

Finally, for $i=1, \cdots, N, Y_{N+4}^{N} \rightarrow Y_{N+4}^{N}+\left|\gamma_{i j}(N)\right|^{2}\left|\gamma_{r}^{D}\right|^{2}\left|\theta_{i j}^{r}\left(u_{i}^{C}, u_{i}^{D}\right)\right|^{2}$ at rate

$$
\begin{cases}\lambda_{r}\left(u_{j}^{N, C}, u_{j}^{N, D}\right) & \text { for } r \in \mathfrak{R}_{D C} \backslash S_{1} \\ \lambda_{r}\left(u_{j}^{N, D}\right) & \text { for } r \in \mathfrak{R}_{D}\end{cases}
$$

Theses transitions admit as debit function

$$
\left|\Psi_{D}^{N}\right|_{j}^{2}\left(u_{C}, u_{D}\right)=\sum_{i=1}^{N}\left|\gamma_{i j}^{N}\right|^{2}|g|_{i j}^{2}\left(u_{C}, u_{D}\right)=:\left|G^{N}\right|_{j}^{2}\left(u_{C}, u_{D}\right)
$$

Furthermore,

$$
\begin{aligned}
\sup _{[0, T]} \sup _{1 \leq k \leq N}\left|Y_{k}^{N}(t \wedge \tau)\right| & =\sup _{[0, T]} \sup _{1 \leq k \leq N}\left|u_{k}^{N}(t \wedge \tau)\right| \\
& \leq c \sup _{[0, T]} \max \left(\left\|u_{C}^{N}(t \wedge \tau)\right\|_{\infty}\left\|u_{D}^{N}(t \wedge \tau)\right\|_{\infty}\right) \\
& \leq c \sup _{[0, T]}\left\|u^{N}(t \wedge \tau)\right\|_{\infty, \infty} \leq C\left(T_{N}, \mu\right)
\end{aligned}
$$

since $u^{N}$ satisfies (34). Therefore, $Y^{N}$ satisfies (34) too, and has a bounded total jump rates when stopped at $\tau$. We then applying Proposition 2.1 of [31] to $Y^{N}$, and the proof is complete.

$\underline{\text { Proof of Lemma } 3.2}$ Set $\varphi_{j}:=\varphi\left(\frac{j}{N}\right)$ for $1 \leq j \leq N$. Since $Z_{C}^{N}$ has the same jumps as $u_{C}^{N}$,

$$
\begin{aligned}
\delta\left\langle Z_{C}^{N}(s), \varphi\right\rangle_{2} & =\delta\left[\frac{1}{N} \sum_{j=1}^{N} Z_{C}^{N}\left(s, \frac{j}{N}\right) \varphi\left(\frac{j}{N}\right)\right] \\
& =\frac{1}{N} \sum_{j=1}^{N} \delta Z_{j}^{N, C}(s) \varphi_{j} \\
& =\frac{1}{N} \sum_{j=1}^{N} \delta u_{j}^{N, C}(s) \varphi_{j} .
\end{aligned}
$$

Recall that $\left[\delta u_{j}^{N, C}(s)\right]\left[\delta u_{k}^{N, C}(s)\right]=0$ for $k \notin\{j-1, j, j+1\}$. Thus,

$$
\begin{aligned}
{\left[\delta\left\langle Z_{C}^{N}(s), \varphi\right\rangle_{2}\right]^{2}=} & \frac{1}{N^{2}} \sum_{j=1}^{N}\left(\left[\delta u_{j-1}^{N, C}(s)\right]\left[\delta u_{j}^{N, C}(s)\right] \varphi_{j-1} \varphi_{j}+\left[\delta u_{j}^{N, C}(s)\right]^{2} \varphi_{j}^{2}\right. \\
& \left.+\left[\delta u_{j}^{N, C}(s)\right]\left[\delta u_{j+1}^{N, C}(s)\right] \varphi_{j} \varphi_{j+1}\right)
\end{aligned}
$$


By Lemma 3.1,

$$
\begin{aligned}
\sum_{s \leq t \wedge \tau}[\delta & \left.\left.\delta Z_{C}^{N}(s), \varphi\right\rangle_{2}\right]^{2} \\
= & \frac{1}{N^{2} \mu} \sum_{j=1}^{N}\left\{\varphi _ { j } ^ { 2 } \int _ { 0 } ^ { t \wedge \tau } \left[N^{2}\left(u_{j+1}^{N, C}(s)+2 u_{j}^{N, C}(s)+u_{j-1}^{N, C}(s)\right)\right.\right. \\
& \left.+\left(|F|_{j}^{2}+\mu\left|F_{1}^{N}\right|_{j}^{2}\right)\left(u^{N}(s)\right)\right] d s \\
& -\varphi_{j} \varphi_{j-1} \int_{0}^{t \wedge \tau} N^{2}\left(u_{j}^{N, C}(s)+u_{j-1}^{N, C}(s)\right) d s \\
& \left.-\varphi_{j} \varphi_{j+1} \int_{0}^{t \wedge \tau} N^{2}\left(u_{j}^{N, C}(s)+u_{j+1}^{N, C}(s)\right) d s\right\}+M(t)
\end{aligned}
$$

where $M(t)$ is a martingale. Therefore, using the 1 -periodicity of our processes w.r.t. the space variable and proceeding to a change of superscript in $j$, we get

$$
\begin{aligned}
\sum_{s \leq t \wedge \tau}[\delta & \left.\left\langle Z_{j}^{N, C}(s), \varphi\right\rangle_{2}\right]^{2} \\
= & \frac{1}{N^{2} \mu} \int_{0}^{t \wedge \tau}\left[\sum_{j=1}^{N} u_{j}^{N, C}(s) N^{2}\left(\varphi_{j-1}^{2}+2 \varphi_{j}^{2}+\varphi_{j+1}^{2}-2 \varphi_{j-1} \varphi_{j}-2 \varphi_{j} \varphi_{j+1}\right)\right. \\
& \left.+\sum_{j=1}^{N}\left(|F|_{j}^{2}+\mu\left|F_{1}^{N}\right|_{j}^{2}\right)\left(u^{N}(s)\right) \varphi_{j}^{2}\right] d s+M(t) \\
= & \frac{1}{N \mu} \int_{0}^{t \wedge \tau}\left[\frac{1}{N} \sum_{j=1}^{N} u_{j}^{N, C}(s)\left(\left[N\left(\varphi_{j-1}-\varphi_{j}\right)\right]^{2}+\left[N\left(\varphi_{j+1}-\varphi_{j}\right)\right]^{2}\right)\right. \\
& \left.+\frac{1}{N} \sum_{j=1}^{N}\left(|F|_{j}^{2}+\mu\left|F_{1}^{N}\right|_{j}^{2}\right)\left(u^{N}(s)\right) \varphi_{j}^{2}\right] d s+M(t) \\
= & \frac{1}{N \mu} \int_{0}^{t \wedge \tau}\left[\left\langle u_{C}^{N}(s),\left(\nabla_{N}^{+} \varphi\right)^{2}+\left(\nabla_{N}^{-} \varphi\right)^{2}\right\rangle_{2}\right. \\
= & \frac{1}{N^{2} \mu} \int_{0}^{t \wedge \tau}\left[\sum_{j=1}^{N} u_{j}^{N, C}(s) N^{2}\left(\varphi_{j-1}^{2}+2 \varphi_{j}^{2}+\varphi_{j+1}^{2}-2 \varphi_{j-1} \varphi_{j}-2 \varphi_{j} \varphi_{j+1}\right)\right. \\
& \left.+\sum_{j=1}^{N}\left(|F|_{j}^{2}+\mu\left|F_{1}^{N}\right|_{j}^{2}\right)\left(u^{N}(s)\right) \varphi_{j}^{2}\right] d s+M(t) \\
= & \frac{1}{N \mu} \int_{0}^{t \wedge \tau}\left[\frac{1}{N} \sum_{j=1}^{N} u_{j}^{N, C}(s)\left(\left[N\left(\varphi_{j-1}-\varphi_{j}\right)\right]^{2}+\left[N\left(\varphi_{j+1}-\varphi_{j}\right)\right]^{2}\right)\right. \\
& \left.+\frac{1}{N} \sum_{j=1}^{N}\left(|F|_{j}^{2}+\mu\left|F_{1}^{N}\right|_{j}^{2}\right)\left(u^{N}(s)\right) \varphi_{j}^{2}\right] d s+M(t) \\
= & \frac{1}{N \mu} \int_{0}^{t \wedge \tau}\left[\left\langle u_{C}^{N}(s),\left(\nabla_{N}^{+} \varphi\right)^{2}+\left(\nabla_{N}^{-} \varphi\right)^{2}\right\rangle_{2}\right. \\
& \left.\left\langle\left(|F|^{2}+\mu\left|F_{1}^{N}\right|^{2}\right)\left(u^{N}(s)\right), \varphi^{2}\right\rangle_{2}\right] d s+M(t) .
\end{aligned}
$$

This shows that (Mg5) defines a martingale. The case of (Mg6) is treated similarly, replacing $|F|^{2}$ by $\left|G^{N}\right|^{2}$. There is no term with the discrete gradient. 


\section{A.4 Regularity and convergence of the debits}

Proof of Proposition 3.1. Denote by $L_{\lambda}$ the Lipschitz constant common to all reactions rates. Fix $u=\left(u_{C}, u_{D}\right), v=$ $\overline{\left(v_{C}, v_{D}\right) \in C_{p}(I) \times C_{p}(I) .}$.

We start with the debit $F$ defined by (16).

$$
\begin{aligned}
& |F(u(x))-F(v(x))| \\
& \quad \leq \sum_{r \in \Re_{C}}\left|\gamma_{r}^{C}\right|\left|\lambda_{r}\left(u_{C}(x)\right)-\lambda_{r}\left(v_{C}(x)\right)\right|+\sum_{r \in S_{1}}\left|\gamma_{r}^{C}\right|\left|\lambda_{r}(u(x))-\lambda_{r}(v(x))\right| \\
& \quad \leq L_{\lambda}\left(\left|u_{C}(x)-v_{C}(x)\right| \sum_{r \in \Re_{C}}\left|\gamma_{r}^{C}\right|+|u(x)-v(x)| \sum_{r \in S_{1}}\left|\gamma_{r}^{C}\right|\right) \\
& \quad \leq L_{\lambda} \bar{\gamma}_{C}\left[\left|u_{C}(x)-v_{C}(x)\right|+\left|u_{D}(x)-v_{D}(x)\right|+\left|u_{D}(x)-v_{D}(x)\right|\right] \\
& \quad \leq L_{F}\left(\left\|u_{C}-v_{C}\right\|_{\infty}+\left\|u_{D}-v_{D}\right\|_{\infty}\right)=L_{F}\|u-v\|_{\infty, \infty} .
\end{aligned}
$$

Thus, $F$ is Lipschitz. The Lipschitz property of the debit $F_{1}^{N}$ given by (17) immediately follows from that of reaction rates, as $F_{1}^{N}$ is a linear combination of some of them.

The function $g$ defined by (19) is Lipschitz. Indeed,

$$
\begin{aligned}
|g(u(x))-g(v(x))| & \leq \sum_{r \in \Re_{D C} \backslash S_{1}}\left|\gamma_{r}^{D}\right|\left|\lambda_{r}(u(x))-\lambda_{r}(v(x))\right|+\sum_{r \in \Re_{D}}\left|\gamma_{r}^{D}\right|\left|\lambda_{r}\left(u_{D}(x)\right)-\lambda_{r}\left(v_{D}(x)\right)\right| \\
& \leq L_{\lambda} \bar{\gamma}_{D}\left[\left|u_{C}(x)-v_{C}(x)\right|+\left|u_{D}(x)-v_{D}(x)\right|+\left|u_{D}(x)-v_{D}(x)\right|\right] \\
& \leq L_{g}\left(\left\|u_{C}-v_{C}\right\|_{\infty}+\left\|u_{D}-v_{D}\right\|_{\infty}\right)=L_{g}\|u-v\|_{\infty, \infty},
\end{aligned}
$$

and $g$ is Lipschitz. As a result, it follows easily that $G$ is Lipschitz.

Recall

$$
G^{N}(u)=\sum_{i, j} \gamma_{i j}^{N} g_{i j}(u) \mathbb{1}_{i}, \text { where } g_{i j}(u)=\sum_{r} \gamma_{r}^{D} \theta_{i j}^{r}\left(u_{i}\right) \lambda_{r}\left(u_{i}\right),
$$

with $j, i=1, \cdots, N$ and $r \in\left(\mathscr{R}_{D C} \backslash S_{1}\right) \cup \mathscr{R}_{D}$. Fix $j, i$, let $x \in I_{i}$ and let $L_{\theta}$ be a Lipschitz constant for the function $\theta$. We successively have

$$
\begin{gathered}
\left|\theta_{i j}^{r}\left(u_{i}\right)-\theta_{i j}^{r}\left(v_{i}\right)\right| \leq\left|\theta\left(u_{i}^{C}+\frac{\gamma_{r}^{C}}{\mu} \gamma_{i j}^{N}\right)-\theta\left(v_{i}^{C}+\frac{\gamma_{r}^{C}}{\mu} \gamma_{i j}^{N}\right)\right| \theta\left(u_{i}^{C}+\gamma_{r}^{D} \gamma_{i j}^{N}\right) \\
+\theta\left(v_{i}^{C}+\frac{\gamma_{r}^{C}}{\mu} \gamma_{i j}^{N}\right)\left|\theta\left(u_{i}^{C}+\gamma_{r}^{D} \gamma_{i j}^{N}\right)-\theta\left(v_{i}^{C}+\gamma_{r}^{D} \gamma_{i j}^{N}\right)\right| \\
\leq L_{\theta}\left(\left|u_{i}^{C}-v_{i}^{C}\right|+\left|u_{i}^{D}-v_{i}^{D}\right|\right) \leq L_{\theta}\|u-u\|_{\infty, \infty}, \\
\left|g_{i j}(u)-g_{i j}(v)\right| \leq \sum_{r}\left|\gamma_{r}^{D}\right|\left[\left|\theta_{i j}^{r}\left(u_{i}\right)-\theta_{i j}^{r}(v i)\right| \lambda_{r}\left(u_{j}\right)+\theta_{i j}^{r}\left(v_{i}\right)\left|\lambda_{r}\left(u_{j}\right)-\lambda_{r}\left(v_{j}\right)\right|\right] \\
\leq L_{\theta} \bar{\gamma} \bar{\lambda}\left(\tilde{\rho}_{T}\right)\|u-v\|_{\infty, \infty}+L_{\lambda} \bar{\gamma}\left|u_{j}-v_{j}\right| \bar{c}\|u-v\|_{\infty, \infty},
\end{gathered}
$$

and it easily follows that $G^{N}$ is Lipschtiz uniformly in $N$.

Since $G^{N}$ and $G$ are Lipschitz with a uniform constant and using a density argument, we may assume that $u=\left(u_{C}, u_{D}\right) \in C^{1}(I) \times C^{1}(I)$. Also, $G$ and $G^{N}$ are continuous w.r.t. $a$ for the $L^{1}$ topology. Indeed, since $\theta$ is bounded by 1 and $g$ is a bounded function

$$
\left\|G^{N}(u)\right\|_{\infty} \leq \bar{\gamma} \bar{\lambda}\left(\tilde{\rho}_{T}\right) \max _{i=1, \ldots, N} \sum_{j=1}^{N} N \int_{I_{j}} \int_{I_{i}} a\left(z-\frac{j}{N}\right) d z d y=\|g\|_{\infty}\|a\|_{L^{1}(I)} .
$$

The same bound holds for $G$ and by linearity, we deduce the continuity property. By density of periodic $C^{1}(I)$ function in $L^{1}(I)$, we may assume that $a \in C^{1}(I)$.

Write for $x \in I_{i}$, and $r$ varying over $\left(\mathscr{R}_{D C} \backslash S_{1}\right) \cup \mathscr{R}_{D}$ :

$$
\left.\left.G^{N}(u)(x)=\sum_{j=1}^{N} \sum_{r} \gamma_{r}^{D} \theta\left(u_{i}^{C}+\frac{\gamma_{r}^{C}}{\mu} \gamma_{i j}^{N}\right)\right) \theta\left(u_{i}^{D}+\gamma_{r}^{D} \gamma_{i j}^{N}\right)\right) N \int_{I_{j}} \lambda_{r}\left(u_{j}\right) \int_{I_{i}} a\left(z-\frac{j}{N}\right) d z d y,
$$


and

$$
G(u)(x)=\sum_{j=1}^{N} \sum_{r} \gamma_{r}^{D} \theta\left(u_{C}(x)\right) \theta\left(u_{D}(x)\right) N \int_{I_{j}} \lambda_{r}(u(y)) \int_{I_{i}} a(x-y) d z d y .
$$

Thus, since $\theta$ is bounded by $1, a$ by $a(0)$ and is a bounded function,

$$
\begin{aligned}
& \left|G^{N}(u)(x)-G(u)(x)\right| \\
& \leq a(0) \sum_{r}\left|\gamma_{r}^{D}\right| \sum_{j=1}^{N} \int_{I_{j}}\left|g\left(u_{j}\right)-g(u(y))\right| d y \\
& \left.\left.\quad+a(0) \bar{\gamma} \bar{\lambda}\left(\tilde{\rho}_{T}\right) \mid \theta\left(u_{i}^{C}+\frac{\gamma_{r}^{C}}{\mu} \gamma_{i j}^{N}\right)\right) \theta\left(u_{i}^{D}+\gamma_{r}^{D} \gamma_{i j}^{N}\right)\right)-\theta\left(u^{C}(x)\right) \theta\left(u^{D}(x)\right) \mid \\
& \quad+\bar{\gamma} \bar{\lambda}\left(\tilde{\rho}_{T}\right) \sum_{j=1}^{N} N \int_{I_{j}} \int_{I_{i}}\left|a\left(z-\frac{j}{N}\right)-a(x-y)\right| d z d y
\end{aligned}
$$

This clearly goes to zero when $N \rightarrow \infty$ thanks to (41) and the Lipschitz property of $\theta, g, a$ and $u$.

\section{A.5 On the Gronwall-Bellman argument}

Proof of Lemma 3.3. From Proposition 2.2 and the observations at the beginning of Section 2.3,

$$
\begin{aligned}
\left\langle\left(\nabla_{N}^{ \pm} T_{N}(t) f\right)^{2}, 1\right\rangle_{2} & =\left\langle T_{N}(2 t) f, \Delta_{N} f\right\rangle_{2} \\
& =\sum_{m}\left[\left.\left\langle\mid f, \varphi_{m, N}\right\rangle_{2}\right|^{2}+\left.\left\langle\mid f, \psi_{m, N}\right\rangle_{2}\right|^{2}\right] \mathrm{e}^{-2 \beta_{m, N} t} \beta_{m, N} \\
& =\sum_{m}\left(\varphi_{m, N}^{2}\left(\frac{j}{N}\right)+\psi_{m, N}^{2}\left(\frac{j}{N}\right)\right) \mathrm{e}^{-2 \beta_{m, N} t} \beta_{m, N} \\
& \leq 2 \sum_{m} \mathrm{e}^{-2 \beta_{m, N} t} \beta_{m, N} .
\end{aligned}
$$

Similarly,

$$
\left\langle\left(T_{N}(t) f\right)^{2}, 1\right\rangle_{2} \leq 1+2 \sum_{m>0} \mathrm{e}^{-2 \beta_{m, N} t}
$$

The result then holds for

$$
h_{N}(t)=1+4 \sum_{m>0} \mathrm{e}^{-2 \beta_{m, N} t}\left(\beta_{m, N}+1\right),
$$

since $\beta_{0, N}=0$ and $\beta_{m, N}>c m^{2}$ for $m>0$ and $c>0$, where $c$ is independent of $m$ and $N$.

Proof of Lemma 3.4. Let $f(x)=e^{x}$ and note

$$
0 \leq f^{\prime \prime}(x+y)=f(x) f(y) \leq 3 f(x) \quad \text { if }|y| \leq 1 .
$$

Using change of variables for functions of bounded variation, we have for $t_{0} \leq t \leq t_{1}$,

$$
\begin{aligned}
f(m(t))= & 1+\int_{t_{0}}^{t} f^{\prime}\left(m\left(s^{-}\right)\right) d m(s) \\
& +\sum_{t_{0} \leq s \leq t}^{t}\left[f(m(s))-f\left(m\left(s^{-}\right)\right)-f^{\prime}\left(m\left(s^{-}\right)\right) \delta m(s)\right] \\
\leq & 1+\int_{t_{0}}^{t} f^{\prime}\left(m\left(s^{-}\right)\right) d m(s)+\frac{3}{2} \sum_{t_{0} \leq s \leq t} f\left(m\left(s^{-}\right)\right)(\delta m(s))^{2},
\end{aligned}
$$

thanks to Taylor's theorem, (ii) of Lemma 3.4 and our observations at the start of the proof. Note that $\int_{t_{0}}^{t} f^{\prime}\left(m\left(s^{-}\right)\right) d m(s)$ has mean 0 and after applying (iii) of Lemma 3.4 and taking expectations, we have

$$
\mathbb{E} f(m(t)) \leq 1+\frac{3}{2} \int_{t_{0}}^{t} \mathbb{E} f(m(s)) h(s) d s .
$$


The result then follows form Gronwall's inequality.

Proof of Lemma 3.5. We want to identify the martingale part of $\sum_{s \leq t}\left[\delta \bar{m}_{C}(t)\right]^{2}$. Integrating $\bar{m}_{C}(t)$ by parts leads to

$$
\begin{aligned}
\bar{m}_{C}(t) & =\left\langle T_{N}(\bar{t}-t) Z_{C}^{N}(t \wedge \tau)+\int_{0}^{t} \Delta_{N} T_{N}(\bar{t}-s) Z_{C}^{N}(s \wedge \tau) d s, f\right\rangle_{2} \\
& =\left\langle T_{N}(\bar{t}-t) Z_{C}^{N}(t \wedge \tau), f\right\rangle_{2}+\left\langle\int_{0}^{t} \Delta_{N} T_{N}(\bar{t}-s) Z_{C}^{N}(s \wedge \tau) d s, f\right\rangle_{2} .
\end{aligned}
$$

Since $t \mapsto \int_{0}^{t} \Delta_{N} T_{N}(\bar{t}-s) Z_{C}^{N}(s \wedge \tau) d s$ is continuous, the corresponding term does not jump and consequently

$$
\delta \bar{m}_{C}(t)=\delta\left\langle T_{N}(\bar{t}-t) Z_{C}^{N}(t \wedge \tau), f\right\rangle_{2}=\left[\delta T_{N}(\bar{t}-t) u_{C}^{N}(t \wedge \tau)\right]_{j^{\prime}}
$$

where $j$ has been fixed within $\{1, \cdots, N\}$. Observing that

$$
\left[T_{N}(\bar{t}-t) u_{C}^{N}(t)\right]_{j}=\sum_{i=1}^{N} u_{i}^{N, C}(t)\left[T_{N}(\bar{t}-t) \mathbb{1}_{i}\right]_{j}
$$

we have

$$
\begin{aligned}
\sum_{j}^{2}= & \left(\sum_{j=1}^{N}\left[\delta u_{i}^{N, C}(t)\right]\left[T_{N}(\bar{t}-t) 1_{i}\right]_{j}\right)^{2} \\
= & \sum_{i=1}^{N}\left(\left[\delta u_{i}^{N, C}(t)\right]^{2}\left[T_{N}(\bar{t}-t) 1_{i}\right]_{j}^{2}\right. \\
& +\left[\delta u_{i}^{N, C}(t)\right]\left[\delta u_{i-1}^{N, C}(t)\right]\left[T_{N}(\bar{t}-t) 1_{i}\right]_{j}\left[T_{N}(\bar{t}-t) 1_{i-1}\right]_{j} \\
& \left.+\left[\delta u_{i}^{N, C}(t)\right]\left[\delta u_{i+1}^{N, C}(t)\right]\left[T_{N}(\bar{t}-t) 1_{i}\right]_{j}\left[T_{N}(\bar{t}-t) 1_{i+1}\right]_{j}\right)
\end{aligned}
$$

We need the forthcoming.

\section{Lemma A.1}

We have $\left[T_{N}(t) \mathbb{1}_{i}\right]_{j}=\left[T_{N}(t) \mathbb{1}_{j}\right]_{i}$ for all $1 \leq i \leq N$.

\section{Proof}

Let $1 \leq i \leq N$ and $t \geq 0$ be fixed. We first remark that

$$
\left\langle\mathbb{1}_{i}, \varphi\right\rangle_{2}=\varphi_{j}=v(j / N), \varphi \in \mathbb{Q}^{N} .
$$

Then, from the spectral decomposition of $\mathrm{T}^{\mathrm{N}}(\mathrm{t})$ on $\left(\mathbb{G}^{N},\langle\cdot, \cdot\rangle_{2}\right)$ (see Proposition 2.2),

$$
\begin{aligned}
j & =\sum_{m} \mathrm{e}^{-\beta_{m, N} t}\left(\left\langle\mathbb{1}_{i}, \varphi_{m, N}\right\rangle_{2} \varphi_{m, N}^{j}+\left\langle\mathbb{1}_{i}, \psi_{m, N}\right\rangle_{2} \psi_{m, N}^{j}\right) \\
& =\sum_{m} \mathrm{e}^{-\beta_{m, N} t}\left(\varphi_{m, N}^{i}\left\langle\mathbb{1}_{j}, \varphi_{m, N}\right\rangle_{2}+\psi_{m, N}^{i}\left\langle\mathbb{1}_{j}, \psi_{m, N}\right\rangle_{2}\right)=\left[T_{N}(t) \mathbb{1}_{j}\right]_{i} .
\end{aligned}
$$


Now, from Lemma 3.1, there exists a martingale $M(t)$ such that, using periodicity and Lemma A.1, we get

$$
\begin{aligned}
& \sum_{s \leq t}\left[\delta T_{N}(\bar{t}-s) u_{C}^{N}(t)\right]_{j}^{2}=\sum_{i=1}^{N} \frac{1}{\mu} \int_{0}^{t \wedge \tau}\left\{\left[N^{2}\left(u_{i+1}^{N, C}(s)+2 u_{i}^{N, C}(s) u_{i-1}^{N}(s)\right)\right.\right. \\
& \left.+\left(|F|_{i}^{2}+\mu\left|F_{1}^{N}\right|_{i}^{2}\right)\left(u^{N}(s)\right)\right]\left[T_{N}(\bar{t}-s) \mathbb{1}_{i}\right]_{j}^{2} \\
& -N^{2}\left(u_{i}^{N, C}(s)+u_{i-1}^{N, C}(s)\right)\left[T_{N}(\bar{t}-s) \mathbb{1}_{i}\right]_{j}\left[T_{N}(\bar{t}-s) \mathbb{1}_{i-1}\right]_{j} \\
& \left.-N^{2}\left(u_{i}^{N, C}(s)+u_{i+1}^{N, C}(s)\right)\left[T_{N}(\bar{t}-s) \mathbb{1}_{i}\right]_{j}\left[T_{N}(\bar{t}-s) \mathbb{1}_{i+1}\right]_{j}\right\} d s+M(t) \\
& =\frac{1}{\mu} \int_{0}^{t \wedge \tau}\left\{\sum _ { i = 1 } ^ { N } u _ { i } ^ { N , C } ( s ) N ^ { 2 } \left(\left[T_{N}(\bar{t}-s) \mathbb{1}_{i-1}\right]_{j}^{2}+2\left[T_{N}(\bar{t}-s) \mathbb{1}_{i}\right]_{j}^{2}+\left[T_{N}(\bar{t}-s) \mathbb{1}_{i+1}\right]_{j}^{2}\right.\right. \\
& -2\left[T_{N}(\bar{t}-s) \mathbb{1}_{i}\right]_{j}\left[T_{N}(\bar{t}-s) \mathbb{1}_{i-1}\right]_{j} \\
& \left.-2\left[T_{N}(\bar{t}-s) \mathbb{1}_{i+1}\right]_{j}\left[T_{N}(\bar{t}-s) \mathbb{1}_{i}\right]_{j}\right) \\
& \left.+\sum_{i=1}^{N}\left(|F|_{i}^{2}+\mu\left|F_{1}^{N}\right|_{i}^{2}\right)\left(u^{N}(s)\right)\left[T_{N}(\bar{t}-s) \mathbb{1}_{i}\right]_{j}^{2}\right\} d s+M(t) \\
& =\frac{1}{\mu N} \int_{0}^{t \wedge \tau}\left\{\frac{1}{N} \sum_{i=1}^{N}\left(N\left[T_{N}(\bar{t}-s) N \mathbb{1}_{j}\right]_{i+1}-\left[T_{N}(\bar{t}-s) N \mathbb{1}_{j}\right]_{i}\right)^{2}\right. \\
& +\left(N\left[T_{N}(\bar{t}-s) N \mathbb{1}_{j}\right]_{i}-\left[T_{N}(\bar{t}-s) N \mathbb{1}_{j}\right]_{i-1}\right)^{2} \\
& \left.+\frac{1}{N} \sum_{i=1}^{N}\left(|F|_{i}^{2}+\mu\left|F_{1}^{N}\right|_{i}^{2}\right)\left(u^{N}(s)\right)\left[T_{N}(\bar{t}-s) N \mathbb{1}_{i}\right]_{j}^{2}\right\} d s+M(t) \\
& =\frac{1}{\mu N} \int_{0}^{t \wedge \tau}\left[\left\langle u_{C}^{N}(s),\left(\nabla_{N}^{+} T_{N}(\bar{t}-s) f\right)^{2}+\left(\nabla_{N}^{-} T_{N}(\bar{t}-s) f\right)^{2}\right\rangle_{2}\right. \\
& \left.+\left\langle\left(|F|^{2}+\mu\left|F_{1}^{N}\right|^{2}\right)\left(u^{N}(s)\right),\left(T_{N}(\bar{t}-s) f\right)^{2}\right\rangle_{2}\right] d s+M(t) .
\end{aligned}
$$

\section{References}

[1] Duncan A, Erban R, Zygalakis K. Hybrid framework for the simulation of stochastic chemical kinetics. J Comput Phys. 2016;326:398-419.

[2] Arnold L. Mathematical models of chemical reactions. In: Hazewinkel M, Williams JC, editor(s). Stochastic systems: the mathematics of filtering and identification and applications,Stochastic Nato Science Series Vol. 78. Dordrecht: D. Reidel Publishing Company. 1980.

[3] Arnold L, Theodosopulu M. Deterministic limit of the stochastic model of chemical reactions with diffusion. Adv Appl Prob. 1980;12:36779.

[4] Belmonte-Beitia ], Woolley TE, Scott ]G, Maini PK, Gaffney EA. Modelling biological invasions: Individual to population scales at interfaces. J Theo Biol, Elsevier. 2013;334:1-12.

[5] Ball K, Kurtz TC, Propovic L, Rempala G. Asymtotic analysis of multiscale approximations to reaction networks. Ann Appl Probab. 2006;16:1925-61.

[6] Blount D]. Comparison of a stochastic model of a chemical reaction with diffusion and the deterministic model. Ph.d., The University of Wisconsin-Madison, 1987.

[7] Blount D]. Law of large numbers in the supremum norm for a chemical reaction with diffusion. Ann Appl Probab. 1992;2:131-41.

[8] Blount D]. Limit theorems for a sequence of nonlinear reaction-diffusion systems. Stochastic Processes Appl. 1993;45:193-203.

[9] Blount D]. Density-dependent limits for a nonlinear reaction-diffusion model. Ann Probab. 1994;22:2040-70.

[10] Buckwar E, Riedler MC. Exact modeling of neuronal membranes including spatio-temporal evolution.] Math Bio. 2011;63:1053-91.

[11] Cintron-Arias A, Banks HT, Capaldi A, Lloyd AL. A sensitivity matrix based methodology for inverse problem formulation. ] Inverse IIIPosed Prob. 2009;15:545-64.

[12] Crudu A, Debussche A, Muller A, Radulescu O. Convergence of stochastic gene networks to hybrid piecewise deterministic processes. Anal Appl Probab. 2012;22:1822-59.

[13] Crudu A, Debussche A, Radulescu O. Hybrid stochastic simplifications for multiscale gene networks. BMC Syst Biol. 2009;3:89.

[14] Cazenave T, Haraux A. An introduction to semilinear evolution equations. Oxford: Clarendon Press, 1998.

[15] Davis MH. Markov models and optimization. In Chapman and London Hall, editors, Monographs on statistics and applied probability, vol. 49, 1993.

[16] Davidson E, Levine M. Gene regulatory networks for development. Proc Natl Acad Sci USA. 2005;102:4936-42

[17] Ethier SN, Kurtz TC.. Markov processes, characterization and convergence. New York: John Wiley and Sons, Inc, 1986 
[18] Érdi P, Thóth ]. Mathematical models of chemical reactions: theory and applications of deterministic and stochastic models. Manchester (U.K.): Nonlinear science, Manchester University Press.

[19] Fange D, Mahmutovic A, Elf]. Mesord 1.0: stochastic reaction-diffusion simulations in the microscopic limit. Bioinformtics 2012;28:31557.

[20] Fu ], Wu S, Li H, Petzold LR. The time dependent propensity function for acceleration of spatial stochastic simulation of reaction diffusion systems. J Comp Phys. 2014;274:524-49.

[21] Cénadot A. Étude multi-échelle de modèles probabilistes pour les systèmes excitables avec composante spatiale. PhD thesis, Université Pierre et Marie Curie 2013.

[22] Cillespie DT. A general method for numerically simulating the stochstic time evolution of coupled chemical reactions. ] Comput Phys. 1976;22:403-34.

[23] Kato T. Perturbation theory for linear operators. Berlin: Springer-Verlag, 1966.

[24] Kotelenez P. Gaussian approximation to the nonlinear reaction-diffusion equation. Report 146, Universität Bremen Forschungsschwerpunkt Dynamische Systemes, 1986.

[25] Kotelenez P. Law of large numbers and central limit theorem for linear chemical reactions with diffusion. Ann Probab. 1986;14:17393.Universität Bremen.

[26] Kotelenez P. Fluctuations near homogeneous states of chemical reaction with diffusion. Adv Appl Probab. 1987;19:352-70.

[27] Kotelenez P. High density limit theorems for nonlinear chemical reactions with diffusion. Probab Theory Related Fields. 1988;78:11-37.

[28] Kotelenez P. University of Ultrecht. Berlin, Heidelberg: Springer. A stochastic reaction-diffusion model 1988.

[29] Kuiper H]. Existence and comparison theorems for nonlinear diffusion systems. ] Math Anal App. 1977;60:166-81.

[30] Kurtz TC. Solutions of ordinary differential equations as limits of pure jump markov processes. ] Appl Prob. 1970;7:49-58.

[31] Kurtz TC. Limit theorems for sequences of jump markov processes approximating ordinary differential processes. ] Appl Prob. 1971;8:344-56.

[32] Li XL, Oduola WO, Qian L, Dougherty ER. Integrating multiscale modeling with drug effects for cancer treatment. Cancer Inf. 2015;14:2131.

[33] MacNeil LT, Walkout A]. Gene regulatory networks and the role of robustness and stochasticity in the control of gene expression. Genome Res. 2011;21:645-57. Cold Spring Harbor Laboratory Press.

[34] A. Noel, K. C. Cheung, and R. Schober. Multi-scale stochastic simulation for diffusive molecular communication. In Communication ICC, London, UK, June 2015. IEEE.

[35] Ortiz AR, Banks HT, Castillo-Chavez C, Chowell G, Wang X. An inverse problem formulation methodology for stochastic models. 2010. https://apps.dtic.mil/docs/citations/ADA556867.

[36] Preziosi L, Teppati G, Bellomo N. Modeling and solution of stochastic inverse problems in mathematical physics. Mathl Comput Modell. 1992;16:37-51.

[37] Radulescu O, Muller O, Crudu A. Théorèmes limites pour des processus de markov à sauts: syntèses de résultats et applications en biologie moléculaire. Tech Sci Inform. 2007;26:443-69.

[38] Riedler MG, Thieullen M, Wainrib G. Limit theorems for infinite-dimensional piecewise deterministic markov processes. applications to stochastic excitable membrane models. Electron ] Probab. 2012;17:1-48.

[39] Spill F, Guerrero P, Alrcon T, Maini PK, Byrne H. Hybrid approches for multiple-species stochastic reaction-diffusion models. ] Comp Phys. 2015;299:429-45.

[40] Schaff]C, Gao F, Li Y, Novak IL, Slepchenko BM.. Spatial deterministic-stochastic models arising in cell biology. PLoS Comput Biol. 2016;12(12):e1005236. https://journals.plos.org/ploscompbiol/article?id=10.1371/journal.pcbi.1005236.

[41] Stathopoulos A, Levine M.. Genomic regulatory networks and animal development. Dev Cell. 2005;9:449-62.

[42] Soubeyrand S, Roques L. Parameter estimation for reaction-diffusion models of biological invasions. Soc Popul Ecol. 2014;56:427-34. 